\title{
SAM JEONG
}

Classificação de segmentos de eletrocardiograma normais e com fibrilação atrial: estudo metodológico 


\title{
SAM JEONG
}

Classificação de segmentos de eletrocardiograma normais e com fibrilação atrial: estudo metodológico

\section{Versão Corrigida}

Dissertação apresentada à Escola Politécnica da Universidade de São Paulo para obtenção do título de Mestre em Ciências

Área de Concentração Engenharia Biomédica

Orientadora: Prof ${ }^{a}$ Dra Cinthia Itiki

\author{
São Paulo
}


Autorizo a reprodução e divulgação total ou parcial deste trabalho, por qualquer meio convencional ou eletrónico, para fins de estudo e pesquisa, desde que citada a fonte.

Este exemplar fol revisado e corrigldo em relaçăo a versăo origlnal, 50 . responsablildade unica do autor e com a anuencla de seu orlentador.

Sằ Paulo, 14 de maio de 2021

Assinatura do autor:

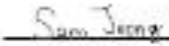

Asslnatura do orlentador:

cotsen

Catalogação-na-publicação

Jeong, Sam

Classificaçăo de segmentos de eletrocardiograma normais e com fibrilação atrial: estudo metodológico / S. Jeong - versão corr. -- São Paulo, 2021. $123 \mathrm{p}$.

Dissertação (Mestrado) - Escola Politécnica da Universidade de São Paulo. Departamento de Engenharia de Telecomunicaçôes e Controle.

1.Fibrilação atrial 2.Processamento de sinais I.Universidade de Săo Paulo. Escola Politécnica. Departamento de Engenharia de Telecomunicaçôes e Controle ll.t. 
Dedico àquele que é meu guia e apoio em todos os momentos. 


\section{AGRADECIMENTOS}

Primeiramente, gostaria de agradecer aos meus pais, que me incentivaram e apoiaram durante a minha formação. Principalmente a minha mãe que infelizmente acabou partindo, durante o decorrer do mestrado.

Aos meus irmãos que tem me dado suporte. Seus incentivos foram fundamentais para que eu não perdesse os prazos de submissão e de apresentação do artigo no congresso.

À Profa. Dra. Cinthia Itiki, por toda a sua paciência e excelente orientação. Apesar de todas as dificuldades e situações durante o período do mestrado, esteve constantemente me orientando e ajudando.

Aos Prof. Dr. Adson Ferreira da Rocha e Prof. Dr. Pai Chi-Nan que contribuíram com sugestões para a versão revisada deste trabalho.

A todos os colegas da Escola Politécnica e do laboratório que tive contato durante o meu mestrado, principalmente a Paula Bezerra Rocha Garcia que colaborou com a preparação do artigo e da apresentação no congresso.

Aos professores da Escola Politécnica, pelas suas aulas ministradas.

A todas as secretárias da pós da Engenharia Elétrica da Escola Politécnica que foram muito atenciosas e solícitas, principalmente a Sra. Elisabete Aparecida de Araujo Fernandes Melo.

À CAPES pelo auxílio financeiro.

A todos os médicos da Santa Casa de Misericórdia que contribuíram para reestabelecimento da minha saúde, para que eu pudesse finalizar o mestrado.

Por fim, a todos os meus amigos, membros da minha igreja e os que direta ou indiretamente contribuíram para a realização deste trabalho. 


\section{RESUMO}

JEONG, Sam. Classificação de segmentos de eletrocardiograma normais e com fibrilação atrial: estudo metodológico. 2021. 123 f. Dissertação (Mestrado em Ciências e Engenharia Biomédica) - Escola Politécnica, Universidade de São Paulo, 2021.

A fibrilação atrial é uma arritmia cardíaca que pode elevar o risco de morte e que está associada ao acidente vascular encefálico e à insuficiência cardíaca. Portanto, sua detecção é muito importante e constitui o objetivo principal deste mestrado. Esta dissertação faz uma revisão dos parâmetros usados para detectar a fibrilação atrial e que são extraídos do eletrocardiograma, do tacograma e da variabilidade da frequência cardíaca. Em seguida, propõe um conjunto de parâmetros que fornece uma alta acurácia nessa tarefa. Esse conjunto de parâmetros é aplicado à entrada de classificadores e fornece uma indicação da presença ou não de fibrilação atrial. Além disso, é feita a comparação entre o desempenho do classificador linear, da máquina de vetor de suporte, da rede neural artificial e da rede convolucional na detecção da fibrilação atrial. Outros aspectos metodológicos como o uso de base de dados distintas, a formação dos grupos de teste e treinamento e a viabilidade da utilização clínica da metodologia também são explorados nesta dissertação. Os resultados indicam que o melhor conjunto de atributos é formado por dez parâmetros obtidos do intervalo RR: a média dos valores absolutos dos ângulos de Poincaré, a média dos senos dos ângulos de Poincaré, o desvio padrão do valor absoluto das diferenças, a mediana dos desvios absolutos entre os intervalos RR e o seu valor médio, a variância, a entropia amostral, a entropia de Fuzzy, a entropia de Shannon, o coeficiente da entropia amostral e a entropia amostral quadrática. Com esses parâmetros normalizados foi obtida até $99,81 \%$ de acurácia com a máquina de vetor de suporte.

Palavras-Chave: Engenharia biomédica. Processamento de sinais. Fibrilação atrial. 


\begin{abstract}
JEONG, Sam. Classification of normal electrocardiogram segments and with atrial fibrillation: methodological study. 2021. 123 f. Dissertação (Mestrado em Ciências e Engenharia Biomédica) - Escola Politécnica, Universidade de São Paulo, 2021.

Atrial fibrillation (AF) is a cardiac arrhythmia that may increase mortality and is associated to stroke and heart failure. Therefore, AF detection is of utmost importance and it is the main objective of this master's research. This thesis reviews the literature and presents parameters used to detect AF, that are extracted from the electrocardiogram, the tachogram and the heart rate variability. Then, it proposes a set of parameters that provide high accuracy in this task. This set of parameters is applied to the input of classifiers and provides an indication of the AF presence or absence. Besides it, the AF detection performances of the linear classifier, the support vector machine, the artificial neural network and the convolutional network are compared. Other methodological aspects such as the use of different data bases, the selection of training and test sets and the feasibility of clinical application are also explored in this master's thesis. Results indicate that the best set of features is given by ten RR-interval parameters: the mean of the absolute values of the Poincaré angles, the mean of the sines of the Poincaré angles, the standard deviation of the absolute differences, the median of the absolute deviations between the RR intervals and theirs average value, the variance, the sample entropy, the Fuzzy entropy, the Shannon entropy, the coefficient of sample entropy and the quadratic sample entropy. Using these normalized parameters, a $99,81 \%$ accuracy was attained with the support vector machine.
\end{abstract}

Keywords: Biomedical engineering. Signal processing. Atrial fibrillation. 


\section{LISTA DE TABELAS}

Tabela 1 Taxas de classificação (SEN-sensibilidade, ESP-especificidade, ACU-acurácia) da fibrilação atrial e parâmetros

Tabela 2 Acurácia (ACU), sensibilidade (SEN) e especificidade (ESP) para as bases de dados MIT-BIH e Long-Term com treinamento pelo método leave-one-subject-out obtidas com o classificador DL

Tabela 3 Acurácia (ACU), sensibilidade (SEN) e especificidade (ESP) para as bases de dados MIT-BIH e Long-Term utilizando as amostras do outro banco de dados para treinamento obtidas com o classificador DL

Tabela 4 Acurácia (ACU), sensibilidade (SEN) e especificidade (ESP) para treinamento pelos métodos de sorteios e de remoção do indivíduo a ser testado (leave-one-subject-out) obtidas com o classificador DL

Tabela 5 Acurácia (ACU), sensibilidade (SEN) e especificidade (ESP) obtidas com o classificador DL e SVM para o treinamento pelo método de leave-one-subject-out

Tabela 6 Acurácia (ACU), sensibilidade (SEN) e especificidade (ESP) na classificação com o treinamento pelo método de leave-one-subjectout e com SVM, com o uso ou não de normalização dos parâmetros

Tabela 7 Acurácia (ACU), sensibilidade (SEN) e especificidade (ESP) na classificação com os parâmetros normalizados e com treinamento pelo método de leave-one-subject-out com SVM, com o uso ou não de sigma único

Tabela 8 Acurácia (ACU), sensibilidade (SEN) e especificidade (ESP) na classificação com os parâmetros normalizados e com treinamento pelo método de leave-one-subject-out para SVM com sigma único e número de ciclos variável

Tabela 9 Acurácia (ACU), sensibilidade (SEN) e especificidade (ESP) para classificações com 60 e 14 parâmetros normalizados como entrada para SVM com sigma único e método de treinamento leave-onesubject-out 
Tabela 10 Classificação com o discriminante linear e treinamento pelo método leave-one-subject-out dos parâmetros cujas acurácias são superiores a $90 \%$

Tabela 11 Classificação com o discriminante linear e treinamento pelo método leave-one-subject-out dos parâmetros cujas acurácias são inferiores a $90 \%$

Tabela 12 Classificação com o discriminante linear e treinamento pelo método leave-one-subject-out dos 14 parâmetros resultantes da eliminação dos parâmetros semelhantes ou iguais da tabela 10

Tabela 13 Classificação com o discriminante linear dos conjuntos de 1 a 14 parâmetros formados a partir dos parâmetros mostrados na tabela 12

Tabela 14 Acurácia (ACU), sensibilidade (SEN) e especificidade (ESP) para diversos conjuntos de parâmetros normalizados como entrada para SVM com o treinamento pelo método leave-one-subject-out

Tabela 15 Acurácia (ACU), sensibilidade (SEN) e especificidade (ESP) para 0 conjunto de 10 parâmetros normalizados como entrada para SVM com os dois métodos de treinamento

Tabela 16 Acurácia (ACU) para treinamentos pelos métodos de sorteios e de remoção do indivíduo a ser testado (leave-one-subject-out) para 19 e 21 neurônios nas camadas internas

Tabela 17 Acurácia (ACU) para treinamentos pelos métodos de sorteios e leaveone-subject-out para rede neural convolucional para paciência de validação igual a 57

Tabela 18 Acurácia (ACU) fornecida na literatura em comparação com a melhor taxa obtida por sorteio dos conjuntos de treinamento e teste 


\section{LISTA DE FIGURAS}

Figura 1

Figura 2

Figura 3

Figura 4

Figura 5

Figura 6

Figura 7

Figura 8

Figura 9

Figura 10 llustração do intervalo RR no eletrocardiograma

Gráfico dos intervalos RR de um segmento com fibrilação atrial (FA) em que os pontos de inflexão estão marcados com o símbolo * em vermelho

Gráfico do número dos intervalos RR em cada grupo de índice $i$ para um segmento com FA

Gráfico de Lorenz para um segmento normal. Os pontos dentro do círculo tracejado e os dentro do círculo contínuo apresentam respectivamente distâncias menores que 0,040 e 0,012 em relação à origem

Gráfico da sequência dos intervalos $R R$ e da respectiva sequência normalizada, para um segmento com fibrilação atrial. A média está representada em linha contínua e as linhas tracejadas representam a média \pm o desvio padrão

Sequência dos intervalos RR da primeira figura 5 filtrada por um filtro do tipo mediana de tamanho 3 e dos seus valores absolutos subtraídos pela mediana. Na figura superior, a média das medianas está representada em linha contínua, a média \pm desvio padrão em linha tracejada. Em ambas as figuras, a respectiva mediana está representada por quadrados

Demonstração da obtenção do valor $V_{2}$ para $k=13$. a) Sequência dos

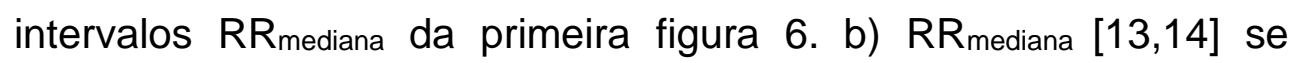
deslocando de $\mathrm{j}=1$ até $\mathrm{j}=\mathrm{N}-3$. c) O valor máximo das diferenças da sequência em a) pelo b)

Sequência dos intervalos RR da primeira figura 5 filtrada por um filtro iterativo. A média está representada pela linha contínua e a média \pm desvio padrão na linha tracejada. A variância obtida foi: 0,0019

Gráfico de Poincaré, reta diagonal e elipse com eixos menor (SD1) e maior (SD2).

Gráfico dos valores discretizados filtrados da sequência mostrada na figura 5 
Figura 11 Gráfico superior representa as diferenças absolutas em relação a mediana. A linha com traços e pontos é o limiar de comparação e corresponde à média dos intervalos RR originais multiplicada por 0,45 . O gráfico inferior mostra os intervalos considerados irregulares, a respectiva média em traço contínuo, mediana em pontilhado e média \pm desvio padrão em tracejado. Ambos os gráficos foram obtidos a partir do sinal original mostrado na figura 5

Figura 12 Exemplo de um gráfico de Poincaré com a reta diagonal com coeficiente angular unitário em linha contínua e a reta tracejada de coeficiente angular -1 que passa pelo ponto $(-2 \mu, 0)$ ou $(0,-2 \mu)$

Figura 13 Exemplo de um gráfico de Poincaré com a reta que define $45^{\circ} \mathrm{em}$ linha contínua e a marcação do ângulo entre a reta que une a origem ao ponto e a reta de $45^{\circ}$ e a marcação da distância do ponto até a origem

Figura 14 Discretização do sinal diferenciado da sequência mostrada na figura 5 e o seu sinal filtrado

Figura 15 Módulo da DFT da sequência mostrada na figura 5

Figura 16 Exemplos de imagens de Poincaré de um segmento com FA à esquerda e de um segmento normal à direita

Figura 17 Exemplo de uma reta de projeção que separa as duas classes representadas por $\boldsymbol{\Delta}$ e $\mathbf{~ m . ~ A ~ r e t a ~ q u e ~ s e p a r a ~ a s ~ c l a s s e s ~ e s t a ́ ~}$ representada pela linha contínua e a reta de projeção pela linha tracejada

Figura 18 Exemplo de um hiperplano de separação, representada com linha contínua, de duas classes representadas por $\boldsymbol{\Lambda}$ e $\mathbf{m}$, com os vetores de suporte em círculos e a margem de separação $\rho$

Figura 19 Esboço de um neurônio com as suas entradas e saída. As entradas $\left[x_{1}, \ldots, x_{n}\right]$ multiplicadas pelos seus respectivos pesos $\left[w_{1}, \ldots, w_{n}\right]$ e o viés $b_{1}$ são introduzidos no neurônio com a função de ativação $F_{1}$ resultando na saída $y$ 
Figura 20 Esboço de uma rede neural do tipo perceptron de múltiplas camadas. Os triângulos representam as entradas $\left[x_{1}, \ldots, x_{n}\right]$ no lado esquerdo $\mathrm{e}$ as saídas $\left[y_{1}, \ldots, y_{m}\right]$ no lado direito. Os círculos representam os neurônios com as suas funções $\left[F_{1}^{1}, \ldots, F_{L}^{S_{L}}\right]$, em que $\left[S_{1}, \ldots, S_{L}\right]$ são a quantidade de neurônios nas camadas $1,2, \ldots, L$ respectivamente

Figura 21 Arquitetura da rede neural implementada com 21 neurônios nas camadas internas

Figura 22 Exemplo de convolução com kernel de tamanho 2x2 com distância entre os centros de valor 1 e sem padding

Figura 23 Exemplo de Avg-Pooling com o resultado da Figura 22 como entrada. O tamanho da unidade de pooling é de $2 \times 2$ com o espaçamento de 1 , ocorrendo sobreposição

Figura 24 Exemplo de Max-Pooling com o resultado da Figura 22 como entrada. O tamanho da unidade de pooling é de $2 \times 2$ com o espaçamento de 1 , ocorrendo sobreposição

Figura 25 Arquitetura da rede de AlexNet

Figura 26 Acurácia obtida por sigma utilizado para banco de dados Long-Term com os parâmetros normalizados e com treinamento pelo método de leave-one-subject-out

Figura 27 Variabilidade por número de componentes principais: de 98,44\% para 1 componente até $100,00 \%$ para 7 ou mais componentes

Figura 28 Acurácia por número de parâmetros como entrada no classificador linear

Figura 29 Acurácia por número de parâmetros como entrada no classificador SVM

Figura 30 Gráfico da acurácia pela paciência da validação da rede neural convolucional treinada com o método de leave-one-subject-out com a imagem de Poincaré como entrada

Figura 31 Esboço do sistema de condução rápida do coração com nodo sinoatrial, nodo atrioventricular, ramo de His e fibras de Purkinje

Figura 32 Ondas típicas do eletrocardiograma normal, com as marcações em verde e a linha de base em azul

Figura 33 Representação da captação do sinal de ECG 
Figura 34 Sinais de a) eletrocardiograma, b) tacograma e c) frequência cardíaca para caso normal.

Figura 35 Sinais de a) eletrocardiograma, b) tacograma e c) frequência cardíaca para caso com fibrilação atrial

Figura 36 Gráfico de representação dos componentes principais 


\section{LISTA DE ABREVIATURAS E SIGLAS}

\begin{tabular}{|c|c|}
\hline ACU & acurácia \\
\hline ApEn & entropia aproximada \\
\hline CA-P & curtose e assimetria das ondas $P$ \\
\hline CC-P & correlação cruzada entre ondas P \\
\hline CE & coeficientes espectrais \\
\hline CNN & rede neural convolucional \\
\hline COSEn & coeficiente da entropia amostral \\
\hline CV & coeficiente de variação \\
\hline CWT-ECG & transformada contínua de wavelet do ECG \\
\hline DP & desvio padrão \\
\hline DL & discriminante linear \\
\hline EB & banda de energia \\
\hline ECG & eletrocardiograma \\
\hline ENLM & rede elástica de modelo logístico \\
\hline ESP & especificidade \\
\hline FA & fibrilação atrial \\
\hline FPG & fotopletismograma \\
\hline FuzzyEn & entropia de Fuzzy \\
\hline HRV & variabilidade da frequência cardíaca \\
\hline ID-P & índice de distorção das ondas $P$ \\
\hline IDA-P & índice de dispersão de amplitude das ondas $\mathrm{P}$ \\
\hline ILSVRC & ImageNet Large Scale Visual Recognition Challenge \\
\hline $\mathrm{K}-\mathrm{NN}$ & K-vizinhos mais próximos \\
\hline LMA & algoritmo de Levenberg-Marquadt \\
\hline LN & classificador linear \\
\hline MAD & mediana do desvio absoluto \\
\hline MAH & Mahalanobis \\
\hline MLP & perceptron de múltiplas camadas \\
\hline PA & pressão arterial \\
\hline QSE & entropia amostral quadrática \\
\hline REB & razão entre energias de bandas \\
\hline
\end{tabular}




$\begin{array}{ll}\text { ReLU } & \text { unidade linear retificadora } \\ \text { RF } & \text { floresta aleatória } \\ \text { RMSSD } & \text { valor eficaz das diferenças sucessivas } \\ \text { RN } & \text { rede neural } \\ \text { SampEn } & \text { entropia amostral } \\ \text { SCG } & \text { sismocardiograma } \\ \text { SEN } & \text { sensibilidade } \\ \text { ShanEn } & \text { entropia de Shannon } \\ \text { SVM } & \text { máquina de vetor de suporte } \\ \text { TPR } & \text { taxa de ponto de inflexão } \\ \text { ARR } & \text { diferenças entre intervalos RR consecutivos } \\ \boldsymbol{\mu} & \text { média } \\ \boldsymbol{\sigma} & \text { desvio padrão }\end{array}$




\section{SUMÁRIO}

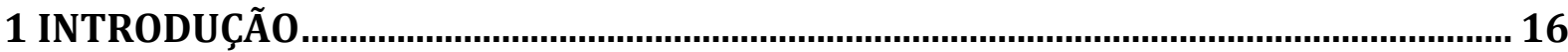

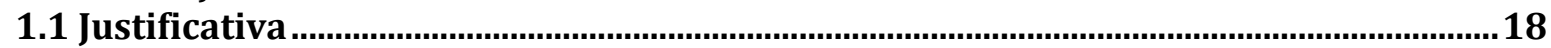

1.2 Objetivo ................................................................................................................18

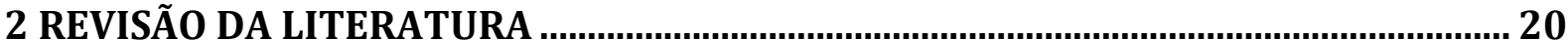

2.1 Sinais para a detecção de fibrilação atrial.....................................................................20

2.2 Parâmetros para a detecção de fibrilação atrial..............................................................21

2.3 Classificadores para a deteç̧ão de fibrilação atrial ........................................................23

2.4 Acurácias na detecção de fibrilação atrial ......................................................................23

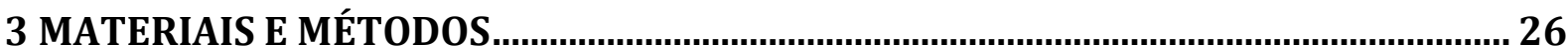

3.1 Banco de dados............................................................................................................26

3.2 Parâmetros ................................................................................................................27

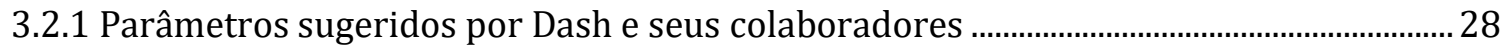

3.2.2 Parâmetros sugeridos por Gilani e seus colaboradores..........................................................30

3.2.3 Parâmetros sugeridos por Islam e seus colaboradores ……………………………………......32

3.2.4 Parâmetros sugeridos por Kennedy e seus colaboradores ........................................................34

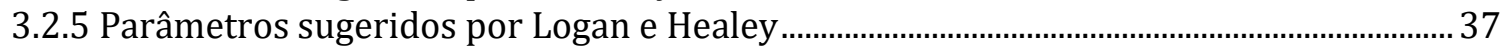

3.2.6 Parâmetros sugeridos por Mabrouki e seus colaboradores....................................................38

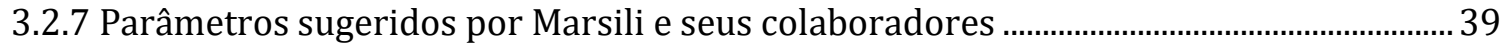

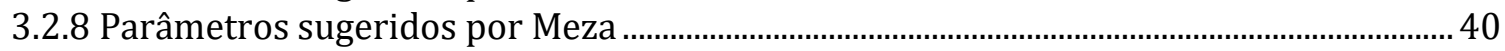

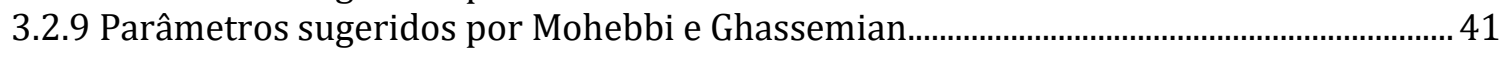

3.2.10 Parâmetros sugeridos por Nuryani e seus colaboradores ...................................................... 43

3.2.11 Parâmetros sugeridos por Slocum e seus colaboradores ..................................................... 43

3.2.12 Parâmetros sugeridos por Trardi e seus colaboradores........................................................ 43

3.2.13 Outros parâmetros........................................................................................................................... 48

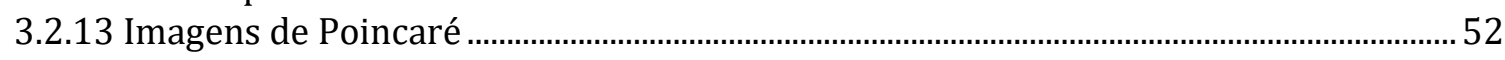

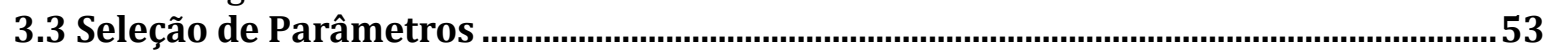

3.4 Discriminante linear de Fisher (DL)_.........................................................................53

3.5 Máquina de vetor de suporte (SVM) ……………….................................................56

3.5.1 Hiperplano de caso linearmente separáveis...............................................................................57

3.5.2 Hiperplano de caso linearmente não-separáveis......................................................................59

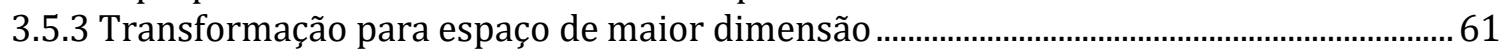

3.6 Rede neural artificial (RNA) ……………………………..................................................62

3.6.1 Modelo matemático dos neurônios (Perceptron)..........................................................................63

3.6.2 Redes neurais de alimentação direta (feedforward).....................................................................64

3.6.3 Método de Backpropagation.................................................................................................65

3.6.4 Treinamento de redes neurais de alimentação direta pelo método de Marquadt..........69

3.7 Rede neural convolucional (CNN do inglês Convolutional Neural Network) ...............71

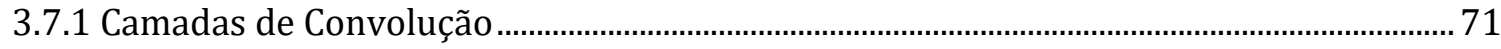

3.7.2 Camadas de Pooling ................................................................................................................................73

3.7.3 Camadas totalmente conectadas (Fully Connected) …………………………………………....74

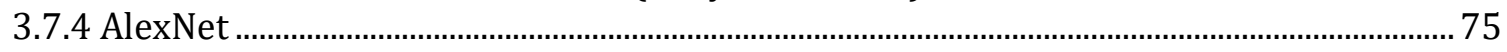

3.7.4.1 Camadas de neurônios retificadores (ReLUs) ………………………………………….....75

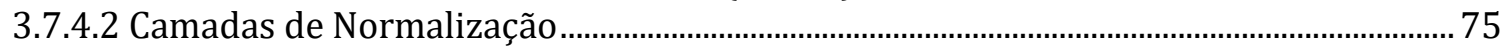

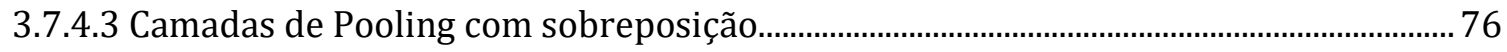

3.7.4.4 Camadas de descarte (dropout) ..........................................................................................76

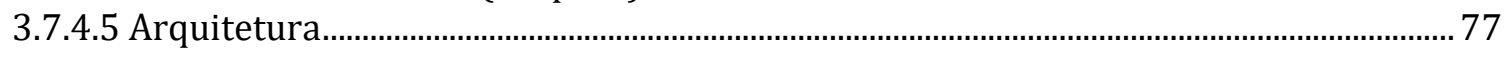

4 RESULTADOS

4.1 Comparação de bancos de dados ...............................................................................

4.2 Influência do método de treinamento no desempenho do discriminante linear .......80

4.3 Comparação entre o discriminante linear e a máquina de vetor de suporte ...............82 
4.4 Influência da normalização no desempenho da máquina de vetor de suporte..........83

4.5 Determinação de uma largura de núcleo única..............................................................84

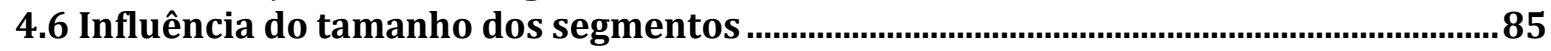

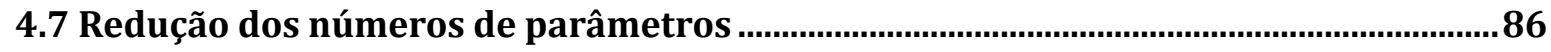

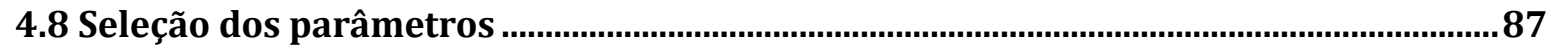

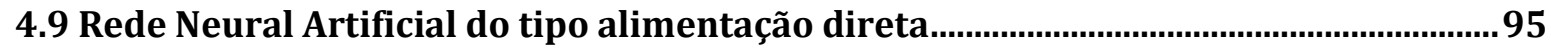

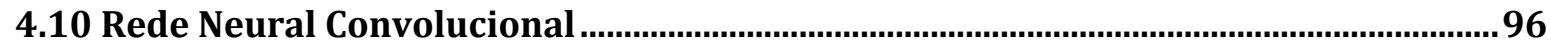

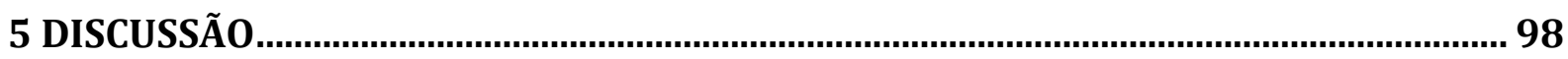

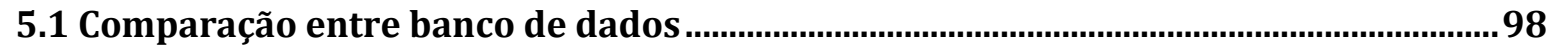

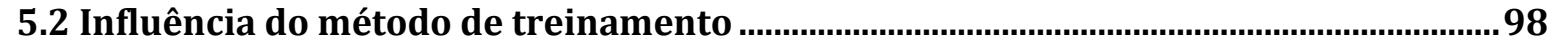

5.3 Influência da normalização no desempenho da máquina de vetor de suporte...........99

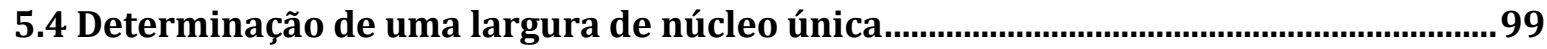

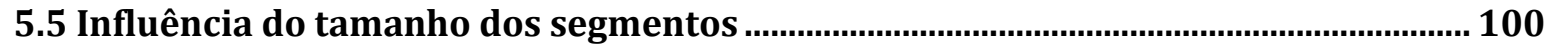

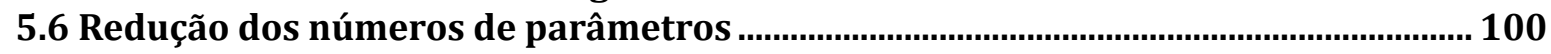

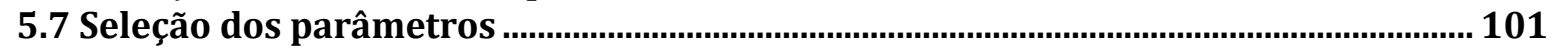

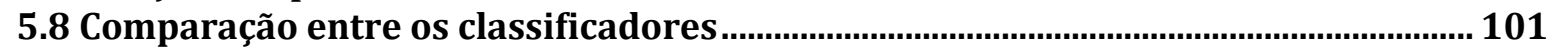

5.8.1 Comparação entre DL e SVM ............................................................................................101

5.8.2 Comparação entre SVM e rede neural artificial do alimentação direta............................102

5.8.3 Comparação da rede neural artificial do tipo alimentação direta com a rede

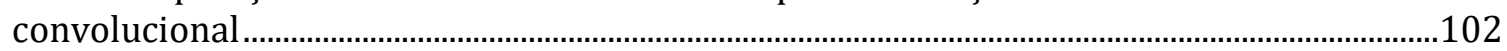

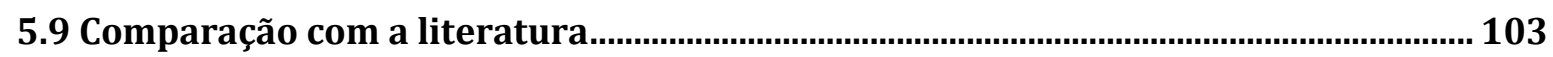

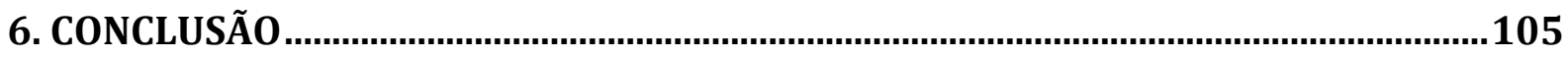

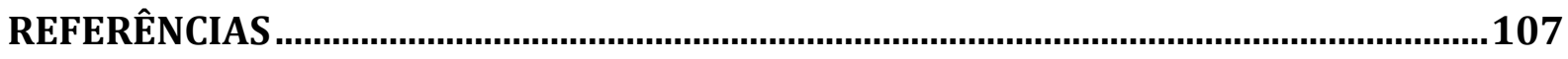

APÊNDICE ...................................................................................................114

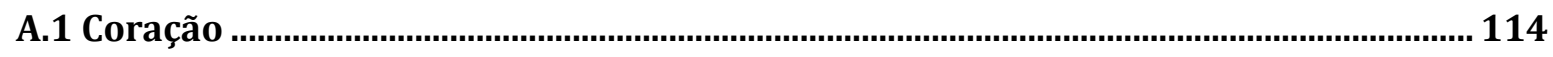

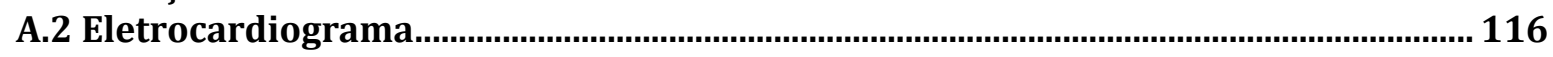

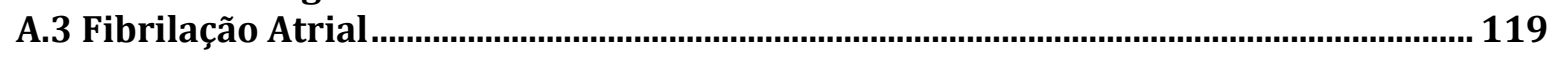

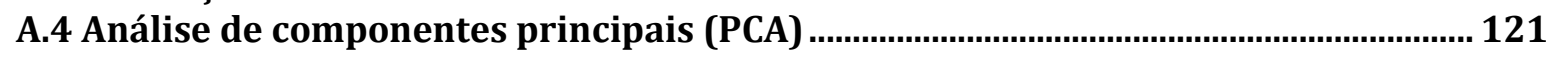




\section{INTRODUÇÃO}

A fibrilação atrial (FA) é uma arritmia cardíaca caracterizada no eletrocardiograma por oscilações de baixa amplitude da linha de base, denominada ondas F, pela elevação e pela irregularidade da frequência cardíaca, além da desorganização da despolarização atrial. (Neto, 2012; Sociedade Brasileira de Cardiologia, 2009)

A detecção da FA é importante, porque ela está associada a diversas patologias (por exemplo, valvopatias, diabetes, hipertensão, acidente vascular encefálico, insuficiência cardíaca) e ao aumento da mortalidade (Sociedade Brasileira de Cardiologia, 2016).

As classificações de FA mais utilizadas são a paroxística, persistente e permanente. A FA é classificada como paroxística quando sua duração é de até 7 dias, sendo revertida de forma espontânea ou com intervenção médica. Quando a sua duração é superior a 7 dias e inferior a 1 ano, a FA é classificada como persistente. Quando a sua duração é superior a 1 ano, é denominada como persistente de longa duração. Quando as tentativas de reversão não são mais realizadas, a FA é considerada permanente. (Sociedade Brasileira de Cardiologia, 2016)

Outra classificação é a de FA isolada que é dada para pacientes com idade inferior a 60 anos e sem fatores de risco ou cardiopatia estrutural, que por estas características, tem baixo risco de complicações tromboembólicas. (Neto, 2012)

O envelhecimento e algumas patologias podem induzir a alterações histológicas e ultraestruturais no miocárdio atrial que, por sua vez, tem um papel importante no início e manutenção da FA (Corradi, 2014).

O diagnóstico rápido da FA se torna importante, pois a própria FA pode induzir alterações estruturais adicionais de forma a realimentar o processo. A remodelação pode chegar ao ponto em que não é possível realizar a reversão ao ritmo normal. Nesse caso, a FA se torna permanente. (Corradi, 2014)

Além da alteração morfológica, também há uma alteração elétrica. No batimento cardíaco normal, o potencial de ação se inicia no nó sinoatrial e se propaga pelos 
átrios até atingir o nó atrioventricular. Essa despolarização dos átrios é representada pela onda $P$ no eletrocardiograma normal. Mas na FA ocorre uma completa desorganização na atividade elétrica atrial. Por isso há uma ausência da despolarização atrial organizada, que pode causar a ausência da onda $P$ no eletrocardiograma e sua substituição por um tremor de alta frequência na linha de base. (Sociedade Brasileira de Cardiologia, 2009)

Apesar de um grande número de estímulos de frequência aleatória atingir o nó atrioventricular, apenas uma parcela deles passa para os ventrículos. A despolarização ventricular é representada pelo complexo QRS no eletrocardiograma. Por isso, a irregularidade na passagem dos potenciais dos átrios para os ventrículos se reflete no intervalo irregular entre os complexos QRS, que é denominado intervalo RR. Com as contrações irregulares do coração, o sangue pode parar de circular, resultando em trombos (coágulos) que, por sua vez, podem levar a ocorrência de acidente vascular encefálico, aumentando a taxa de mortalidade. (Sociedade Brasileira de Cardiologia, 2009)

Nos casos em que o paciente também apresente bloqueio ventricular total ou taquicardia juncional, pode ocorrer a presença do tremor da linha de base com ausência da onda $\mathrm{P}$, mas com a regularidade no intervalo entre os complexos QRS regulares. (Sociedade Brasileira de Cardiologia, 2009)

A FA apresenta uma taxa de prevalência alarmante. Nos Estados Unidos 3,2 milhões de pessoas apresentaram FA na década de 1980 (Zipes et al, 2018). No Brasil, estimase que a prevalência da FA seja de 1,5\% da população, em 2016 (de Moraes et al, 2019). E a FA está presente em aproximadamente $2 \%$ da população adulta mundial (Potpara; Lip, 2015).

Sabe-se que esse número aumenta com o envelhecimento da população. Por isso, 15\% dos idosos acima de 80 anos apresentam FA (Potpara; Lip, 2015). As projeções indicam que, em 2050, 15,9 milhões de norte-americanos (Zipes et al, 2018) e 1,7\% dos brasileiros apresentarão FA (de Moraes et al, 2019). 
Além disso, um a cada três pacientes com FA necessita ser hospitalizado (Zipes et al, 2018). No caso dos EUA o número de internações associadas à FA atingiu 5,1 milhões em 2000 (Zipes et al, 2018).

O diagnóstico da FA pode indicar desde uma cardioversão elétrica ou farmacológica até uma intervenção cirúrgica, com o objetivo de manter o ritmo sinusal e melhorar a qualidade de vida. Por isso, seu diagnóstico precoce se torna importante (Sociedade Brasileira de Cardiologia, 2009; Sociedade Brasileira de Cardiologia, 2016).

\subsection{Justificativa}

Há vários trabalhos na literatura com vários parâmetros, tipos de classificadores e bases de dados que resultam em altas taxas de classificação. (Trardi et al, 2018; Xia et al, 2018)

Porém, não há uma comparação sistemática desses parâmetros, classificadores e bases de dados, já que cada trabalho usa uma metodologia diferente.

Portanto, este mestrado se propõe a fazer uma análise comparativa dos parâmetros, dos classificadores e até mesmo da influência das bases de dados nos resultados.

\subsection{Objetivo}

Este mestrado tem como objetivo analisar a influência dos parâmetros, métodos de treinamento, bases de dados e classificadores na detecção da fibrilação atrial.

Primeiramente, para atingir esse objetivo é apresentada uma pesquisa bibliográfica dos parâmetros utilizados na literatura para a detecção da fibrilação atrial, obtidos a partir do eletrocardiograma, do tacograma e da frequência cardíaca.

Em seguida, propõe-se uma combinação dos parâmetros a serem usados para se obter as melhores taxas de classificação.

Após a seleção dos parâmetros, são analisados os desempenhos de diversos classificadores: discriminante linear, máquina de vetor de suporte de base radial, uma rede neural artificial clássica e uma rede convolucional. 
Além disso são investigados os efeitos do método de treinamento no desempenho desses classificadores.

Neste mestrado, também é discutida a influência da escolha da base de dados nos resultados da detecção de FA.

E, por fim, os resultados obtidos são comparados com os de outros métodos estabelecidos na literatura. 


\section{REVISÃO DA LITERATURA}

Há diversos tipos de arritmias cardíacas como a insuficiência cardíaca, o flutter atrial ou ventricular, o bloqueio dos ramos direito ou esquerdo, a bradicardia sinusal, a contração ventricular prematura e o bloqueio de segundo grau, que são detectados por uma variedade de técnicas de processamento (Yoon et al., 2015; Maji et al., 2016; Kelwade; Salankar, 2016).

Especificamente para o caso da FA, há dispositivos em hardware que a detectam. Recentemente, estão sendo desenvolvidos circuitos e dispositivos portáteis ou vestíveis que realizam a detecção da FA. (Andersson et al, 2015; Chong et al, 2015; Nemati et al, 2016).

Este capítulo apresenta uma revisão dos sinais biológicos e dos parâmetros derivados desses sinais que são usados na deteç̧ão de FA. Também indica os classificadores e seus desempenhos, conforme registrado na literatura.

\subsection{Sinais para a detecção de fibrilação atrial}

Há técnicas de software que podem ser aplicadas a diversos sinais cardíacos, com o objetivo de detectar a FA. Os algoritmos têm sido aplicados aos sinais de sismografia (Humanen et al, 2017), fotopletismografia (Shan et al, 2016), PA (Wiesel et al, 2014; Verberk et al, 2016; Meža, 2015) além do eletrocardiograma (Shan et al, 2016).

Os sinais derivados do eletrocardiograma (ECG) são os mais usados na detecção de FA. As técnicas de processamento são aplicadas basicamente aos seguintes sinais:

- ao ECG em si (Slocum et al, 1992; Sheikh et al, 2015; Xia et al, 2018);

- aos intervalos entre picos R consecutivos, denominados intervalos RR (Kalsi; Prakash, 2016; Moody et al, 1983; Logan; Healey, 2005; Mohebbi; Ghassemian, 2012; Ladavich; Ghoraani, 2014; Pürerfellner et al, 2014; Maji; Mitra; Pal, 2014; Hargittai, 2014; Kennedy et al, 2016; Linker et al, 2016; Islam et al, 2016; Mabrouki; Khaddoumi; Sayadi, 2016; Yoon et al, 2015; Andersson et al, 2015; Nemati et al, 2016); 
- às diferenças entre intervalos $R R$ consecutivos, representados por $\Delta R R$ (Dash et al, 2009; Hargittai, 2014; Nuryani et al, 2014; Andersson et al, 2015; Kennedy et al, 2016; Shan et al, 2016) e

- às ondas $P$ (ou intervalos entre o fim das ondas $T$ e o início dos complexos QRS). [Kalsi; Prakash, 2016; Pürerfellner et al, 2014; Ladavich; Ghoraani, 2014; Censi et al, 2016; García et al, 2016]

\subsection{Parâmetros para a detecção de fibrilação atrial}

Uma das características da FA é a irregularidade da frequência cardíaca. Por isso, os parâmetros que detectam a alteração da frequência cardíaca como os batimentos irregulares podem ser utilizados para detectar a FA. Alguns parâmetros que indicam a irregularidade da frequência cardíaca seriam aplicados aos intervalos $R R$ ou às diferenças $\Delta R R$ entre intervalos:

- variância (Logan; Healey, 2005; Ladavich; Ghoraani, 2014);

- valor eficaz das diferenças sucessivas (Dash et al, 2009; Andersson et al, 2015; Kennedy et al, 2016; Shan et al, 2016);

- desvio padrão (Maji; Mitra; Pal, 2014; Meža, 2015; Nuryani et al, 2015; Kalsi; Prakash, 2016; Shan et al, 2016);

- coeficiente de variação (Kennedy et al, 2016);

- mediana do desvio absoluto (Kennedy et al, 2016);

- taxa de ponto de inflexão (Dash et al, 2009; Shan et al, 2016; Andersson et al, 2015; Chong et al, 2015; Pürerfellner et al, 2014);

- gráficos de Poincaré, dos intervalos RR atuais versus os intervalos anteriores (Mohebbi; Ghassemian, 2012; Mabrouki; Khaddoumi; Sayadi, 2016; Chong et al, 2015);

- gráficos de Lorenz, das atuais diferenças entre intervalos RR consecutivos versus as anteriores (Hargittai, 2014).

A dissimilaridade entre os intervalos $\mathrm{RR}$ ou vetores de dois intervalos consecutivos, que também é um indicador de FA, poderia ser estimada pelos seguintes parâmetros: 
- entropia amostral (Mohebbi; Ghassemian, 2012; Mabrouki; Khaddoumi; Sayadi, 2016; Hargittai, 2014; Kennedy et al, 2016; Yoon et al, 2015; Nemati et al, 2016);

- entropia de Shannon (Dash et al, 2009; Shan et al, 2016; Yoon et al, 2015; Andersson et al, 2015; Chong et al, 2015).

Além da irregularidade da frequência cardíaca, outra característica da FA é a elevação dessa frequência. Os parâmetros que estimam o aumento da frequência cardíaca seriam:

- média (Linker et al, 2016; Islam et al, 2016; Nuryani et al, 2015; Meža, 2015);

- mediana (Linker et al, 2016; Islam et al, 2016, Shan et al, 2016).

Os parâmetros do domínio da frequência, assim como os do domínio do tempo, também podem indicar a irregularidade da frequência cardíaca. A existência de picos de amplitudes em frequências distintas poderia ser um indício dessa irregularidade. Por isso, os seguintes parâmetros do domínio da frequência também têm sido usados na detecção da FA:

- coeficientes espectrais do ECG (Xia et al, 2018);

- coeficientes wavelet do ECG (García et al, 2016; Xia et al, 2018);

- razão entre a energia numa banda específica e a energia total do espectro do ECG (Slocum et al, 1992; Sheikh et al, 2015; Shan et al, 2016);

- frequência do pico máximo do espectro do ECG (Sheikh et al, 2015);

- energia de uma banda específica do espectro do ECG (Shan et al, 2016);

- coeficientes do biespectro entre ciclos consecutivos do ECG (Mohebbi e Ghassemian, 2012).

Além da irregularidade na frequência cardíaca na $F A$, há um tremor de alta frequência da linha de base do ECG, que substitui as ondas $P$ regulares. Por isso, há parâmetros que verificam a existência ou não de ondas $P$ normais e que podem ser utilizados para detectar a FA.

- ausência da onda P (Kalsi; Prakash, 2016; Pürerfellner et al, 2014);

- valor médio da onda P (Ladavich; Ghoraani, 2014); 
- curtose e assimetria da onda P (Ladavich; Ghoraani, 2014);

- correlação cruzada entre as ondas P (Censi et al, 2016);

- índice de dispersão de amplitude das ondas $\mathrm{P}$ (Censi et al, 2016);

- índice de distorção das ondas P (Censi et al, 2016).

\subsection{Classificadores para a detecção de fibrilação atrial}

Os parâmetros citados acima servem de entrada aos classificadores. Por sua vez, os classificadores é que realizam a detecção de FA. Os classificadores podem ser do tipo lineares, por distância de Mahalanobis (Ladavich; Ghoraani, 2014), K-vizinhos mais próximos (Kennedy et al, 2016), máquinas de vetor de suporte (Nuryani et al, 2015; Meža, 2015; Shan et al, 2016), redes neurais artificiais (Meža, 2015), árvores de decisão e florestas aleatórias (Meža, 2015; Kennedy et al, 2016), por exemplo.

Mais recentemente os classificadores de aprendizado profundo (deep-learning) como a rede neural convolucional (CNN) e rede neural residual (ResNet) estão sendo implementados para detecção da fibrilação atrial. Esses classificadores apresentam várias camadas internas permitindo que o próprio classificador obtenha os parâmetros a partir das entradas e com elas realizar as classificações.

A CNN é utilizada normalmente com imagens na entrada. Nas suas camadas internas cada neurônio está ligado a todos os neurônios da camada seguinte. (Guo et al, 2019; Mashrur; Roy; Saha, 2019; Singh et al, 2019)

Por outro lado, na ResNet cada camada interna está conectada à camada posterior e a algumas camadas anteriores. Dessa forma, os resíduos das camadas anteriores influenciam nas camadas posteriores. (Jalali; Lee, 2020)

\subsection{Acurácias na detecção de fibrilação atrial}

Dentre os artigos mencionados na revisão da literatura, as acurácias obtidas estavam na faixa de $85,92 \%$ (Meža, 2015) a 99,77\% (Trardi et al, 2018b). Alguns outros artigos mencionam também as sensibilidades e especificidades. As sensibilidades variaram entre $87,8 \%$ (Slocum et al, 1992) e 99,97\% (Trardi et al, 2018b). As especificidades ficaram na faixa de $68,3 \%$ (Slocum et al,1992) a 99,3\% (Trardi et al, 2018). 
Apesar dos classificadores mais recentes como a ResNet e CNN apresentarem acurácias elevadas de 89,2\% e 98,63\% (Jalali; Lee, 2020; Xia et al, 2018), os classificadores mais simples como a SVM também apresentam taxas de classificações de até 99,77\% (Trardi et al, 2018b).

A tabela 1 a seguir resume os sinais, os parâmetros, os classificadores e as taxas de classificação apresentadas nesta revisão bibliográfica. 
Tabela 1 - Taxas de classificação (SEN-sensibilidade, ESP-especificidade, ACU-acurácia) da fibrilação atrial e parâmetros.

\begin{tabular}{|c|c|c|c|c|c|c|}
\hline Autores & Sinal & Parâmetros & Classificador & SEN & ESP & ACU \\
\hline Moody et al., 1983 & RR & Processo Markov & LN & $96,09 \%$ & $86,79 \%$ & \\
\hline Slocum et al., 1992 & ECG & REB & LN & $87,8 \%$ & $68,3 \%$ & \\
\hline Logan e Healey, 2005 & RR & Variância & LN & $96 \%$ & $89 \%$ & \\
\hline Dash et al., 2009 & $\Delta \mathrm{RR}$ & RMSSD, TPR, ShanEn & LN & $90,2 \%$ & $91,2 \%$ & \\
\hline Mohebbi e Ghassemian, 2012 & RR & Poincaré, SampEn, Biespectro & SVM & $96,3 \%$ & $93,1 \%$ & \\
\hline Ladavich e Ghoraani, 2014 & RR, $P$ & Variância, Média, CA-P & MAH & $99,28 \%$ & $90,21 \%$ & \\
\hline Pürerfellner et al., 2014 & RR & TPR, Ausência da onda P & LN & $97,8 \%$ & $99,3 \%$ & \\
\hline Maji, Mitra e Pal, 2014 & RR & DP & LN & $96,30 \%$ & $96,09 \%$ & \\
\hline Hargittai, 2014 & RR & Lorenz, SampEn & LN & $93,93 \%$ & $96,56 \%$ & \\
\hline Nuryani et al., 2015 & $\Delta \mathrm{RR}$ & DP, Média & SVM & $95,81 \%$ & $98,44 \%$ & $97,50 \%$ \\
\hline Sheikh et al., 2015 & ECG & REB, Frequência do pico máximo & LN & & & $98 \%$ \\
\hline Kennedy et al., 2016 & $\mathrm{RR}, \Delta \mathrm{RR}$ & RMSSD, CV, MAD, SampEn & LN, RF, K-NN & $92,8 \%$ & $98,3 \%$ & \\
\hline Censi et al., 2016 & $\mathrm{P}$ & CC-P, IDA-P, ID-P & LN & $98,6 \%$ & $95,0 \%$ & \\
\hline Linker et al., 2016 & RR & Média, Mediana & LN & $98,0 \%$ & $98,2 \%$ & \\
\hline Islam et al., 2016 & RR & Média, Mediana & LN & & & $96,38 \%$ \\
\hline Mabrouki, Khaddoumi e Sayadi, 2016 & RR & Poincaré, SampEn & LN & $99,65 \%$ & $99,33 \%$ & \\
\hline Xia et al., 2018 & ECG & CE, Wavelet & CNN & $98,79 \%$ & $97,87 \%$ & $98,63 \%$ \\
\hline Meža, 2015 & PA & DP, Média & $\begin{array}{l}\text { RF, SVM, } \\
\text { RNA }\end{array}$ & & & $85,92 \%$ \\
\hline Wiesel et al., 2014 & PA & & & $97 \%$ & $92 \%$ & \\
\hline Verberk et al., 2016 & PA & & & $99 \%$ & $93 \%$ & \\
\hline Hurnanen et al., 2017 & SCG & Entropia Espectral, HRV & LN & $99,9 \%$ & $96,4 \%$ & \\
\hline Shan et al., 2016 & $\Delta \mathrm{RR}$ & RMSSD, DP, TPR, Med, REB, EB, ShanEn & SVM & $94,2 \%$ & $96,2 \%$ & $95,7 \%$ \\
\hline Yoon et al., 2015 & RR & SampEn, ShanEn & LN & $95,22 \%$ & $96,49 \%$ & $96,07 \%$ \\
\hline Maji et al., 2016 & RR, ECG & DP, Duração RR & LN & $98,24 \%$ & $96,08 \%$ & \\
\hline Kelwade e Salankar, 2016 & RR & Duração RR & RN & & & $96,33 \%$ \\
\hline Andersson et al., 2015 & $\mathrm{RR}, \Delta \mathrm{RR}$ & RMSSD, SampEn, ShanEn & LN & $94,9 \%$ & $95,8 \%$ & \\
\hline Chong et al., 2015 & $\mathrm{RR}^{*}$ & RMSSD, TPR, Poincaré, ShanEn & LN & $98,86 \%$ & & $96,26 \%$ \\
\hline Chong et al., 2016 & $\mathrm{RR}^{*}$ & RMSSD, TPR, Poincaré, ShanEn & SVM & $96,67 \%$ & $97,65 \%$ & $96,67 \%$ \\
\hline Nemati et al., 2016 & RR, FPG & SampEn, DP & ENLM & $97 \%$ & $94 \%$ & $95 \%$ \\
\hline Trardi et al, 2018b & $\mathrm{RR}, \Delta \mathrm{RR}$ & $\begin{array}{l}\text { Média, RMS, Curtose, Assimetria, Mediana, } \\
\text { Poincaré, Lorenz, SampEn, ApEn, FuzzyEn, } \\
\text { COSEn, QSE, RMSSD }\end{array}$ & SVM & $99,97 \%$ & $99,60 \%$ & $99,77 \%$ \\
\hline Mashrur et al, 2019 & ECG & CWT & CNN & $98,65 \%$ & $92,86 \%$ & $97,94 \%$ \\
\hline
\end{tabular}

Observação: ECG (eletrocardiograma), SCG (sismocardiograma), FPG (Fotopletismograma), PA (pressão arterial), RR (intervalo entre os picos R), $\triangle R R$ (diferenças entre intervalos RR consecutivos), CWT (Transformada contínua de wavelet); EB (energia de banda), REB (razão entre energias de bandas), CE (coeficientes espectrais), RMSSD (valor eficaz das diferenças sucessivas), CV (coeficiente de variação), DP (desvio padrão), MAD (mediana do desvio absoluto), TPR (taxa de ponto de inflexão), ShanEn (Entropia de Shannon), SampEn (Entropia amostral), ApEn (Entropia aproximada), FuzzyEn (Entropia de

Fuzzy), COSEn (Coeficiente da entropia amostral), QSE (Entropia amostral quadrática), CA-P (curtose e assimetria das ondas P), CC-P (correlação cruzada entre ondas P), IDA-P (índice de dispersão de amplitude das ondas P), ID-P (índice de distorção das ondas P); HRV (variabilidade da frequência cardíaca), LN (classificador linear), SVM (máquina de vetor de suporte), RNA (Rede Neural Artificial), RF (floresta aleatória), K-NN (K-vizinho mais próximo), ENLM (rede elástica de modelo logístico), MAH (Mahalanobis), CNN (Rede Neural Convolucional) 


\section{MATERIAIS E MÉTODOS}

Nesse capítulo são descritos os bancos de dados, os parâmetros e os classificadores estudados.

\subsection{Banco de dados}

Neste trabalho foram utilizados dois bancos de dados, o primeiro deles é o banco de dados Long-Term Atrial Fibrillation da Physionet. Esse banco de dados contém 84 arquivos ".dat" com os sinais das derivações MLII e V1 modificada de eletrocardiogramas amostrados a $128 \mathrm{~Hz}$, de duração típica entre 24 e 25 horas. Nos arquivos '.atr' correspondentes, há a marcação dos picos $\mathrm{R}$, do tipo de batimento e das mudanças dos ritmos cardíacos que ocorrem nos sinais. (Petrutiu; Sahakian; Swiryn, 2007; Goldberger et al, 2000)

As marcações dos QRS fornecidas no banco de dados foram inicialmente obtidas de forma automatizada e posteriormente validadas por uma equipe de especialistas em ECG da empresa MEDICALgorithmics Ltd (Warsaw, Poland). Além disso, as anotações da fibrilação atrial foram feitas por dois médicos (Physionet, 2008).

Os arquivos '.atr' podem apresentar até 6 variáveis. Dentre essas variáveis, as mais importantes são: a localização das anotações em amostras (primeira variável), a descrição do tipo de batimento (segunda variável) e o ritmo a que pertence este batimento (última variável).

De cada sinal do arquivo '.dat' foram utilizadas as primeiras 4 milhões de amostras. As marcações dos picos $\mathrm{R}$ do arquivo 'atr' correspondente foram utilizadas como início e fim de um ciclo cardíaco. Cada sinal foi dividido em segmentos com 60 ciclos cardíacos. Foram selecionados segmentos com ritmo normal ou com fibrilação atrial. Os demais segmentos foram descartados.

Também foram eliminados os segmentos que apresentavam algum intervalo entre os picos $\mathrm{R}$ menor que 0,3 segundos ou maior que 2 segundos, de forma a evitar os trechos que apresentavam perda de sinal ou possíveis marcações erradas. Além disso os segmentos com batimentos anormais também foram descartados. 
Foram selecionados ao todo 35275 segmentos para cada sinal do eletrocardiograma sendo 12866 normais e 22409 com fibrilação atrial.

Além deste banco de dados, também foi utilizado o banco de dados MIT-BIH Atrial Fibrillation, que contém 23 arquivos '.dat' com sinais de eletrocardiograma de derivação MLII e V1 modificada (ou V1), amostrados a 250 Hz. Nos arquivos '.atr' equivalentes há a marcação dos picos $R$, do tipo de batimento e das mudanças dos ritmos cardíacos. Os arquivos 00735 e 03665 não foram considerados, porque não apresentavam o eletrocardiograma, mas apenas o tacograma. (Moody; Mark, 1983; Goldberger et al, 2000)

As marcações dos QRS fornecidas no banco de dados foram obtidas de forma automatizada. Apenas alguns sinais apresentaram correções manuais inseridas por especialistas. Por outro lado, todas as anotações dos ritmos foram feitas manualmente. (Moody; Mark, 1983)

Também foram aplicados os mesmos procedimentos de segmentação utilizados no banco de dados anterior, sendo obtidos, ao todo, 16396 segmentos. Destes, 8994 são normais e 7402 com fibrilação atrial.

\subsection{Parâmetros}

Foi realizada uma busca na literatura dos parâmetros extraídos do eletrocardiograma e utilizados na detecção da FA. A utilização de parâmetros extraídos da onda $P$ foi descartada por três motivos: as acurácias na classificação eram baixas, a descrição da metodologia para determinar a presença ou ausência da onda $P$ não era muito detalhada e a determinação do início da onda P era difícil.

Foram, então, considerados os parâmetros obtidos do espectro do sinal de eletrocardiograma e dos intervalos RR. Esses intervalos são determinados pelos intervalos de tempo entre picos $\mathrm{R}$ consecutivos, conforme ilustrado na figura 1. 
Figura 1: llustração do intervalo RR no eletrocardiograma.

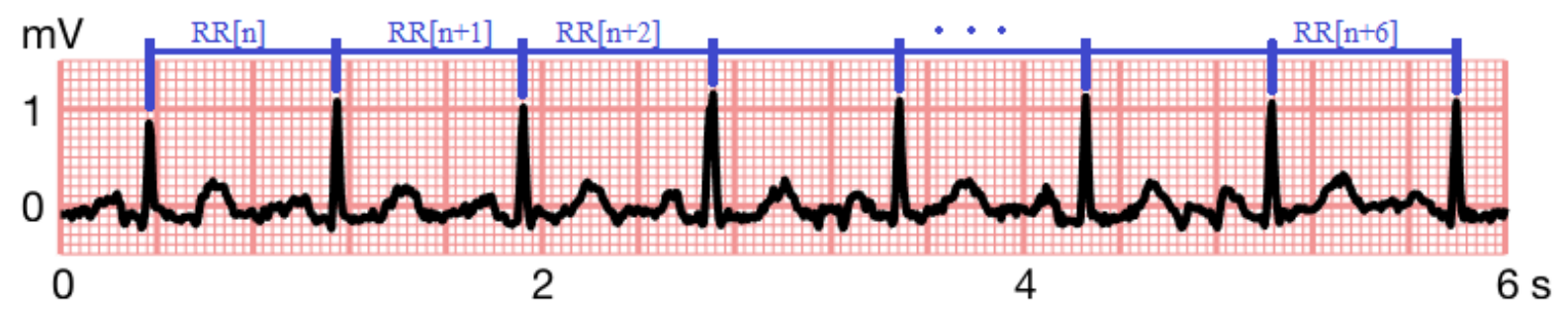

Fonte: Próprio autor.

A descrição dos parâmetros analisados é fornecida a seguir.

\subsubsection{Parâmetros sugeridos por Dash e seus colaboradores}

Os parâmetros sugeridos por Dash e seus colaboradores (2009) foram computados para os intervalos RR: a raiz da média quadrática das diferenças, a entropia de Shannon e a taxa de ponto de inflexão.

A raiz da média quadrática das diferenças (RMSSD) é calculada por

$$
\operatorname{RMSSD}=\sqrt{\sum_{n} \frac{(R R[n+1]-R R[n])^{2}}{N-1}},
$$

para $n=1,2, \ldots, N-1$; em que $\mathrm{N}=60$ intervalos e $R R[n]$ é o n-ésimo intervalo RR.

A taxa de ponto de inflexão (TPR) é baseada no número de mínimos e máximos locais da sequência de intervalos RR. Em uma sequência aleatória de comprimento $L$, o número esperado de mínimos e máximos locais é de $(2 L-4) / 3$, com desvio padrão de $\sqrt{(16 L-29) / 90}$. Quando o número de máximos e mínimos locais estiver dentro de uma faixa esperada, o valor da TPR é igualado a 1 e caso contrário, a 0. 
Figura 2: Gráfico dos intervalos RR de um segmento com fibrilação atrial (FA) em que os pontos de inflexão estão marcados com o símbolo * em vermelho.

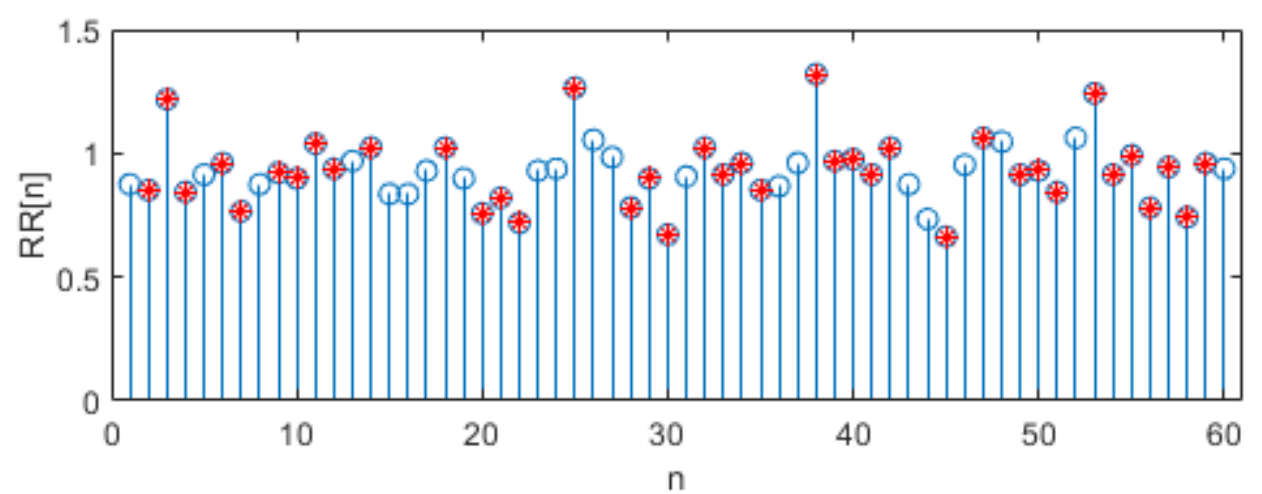

Fonte: Próprio autor.

A entropia de Shannon é calculada com valores discretizados. Para calcular a entropia de Shannon, são eliminados os 8 maiores e os 8 menores valores dos intervalos RR. Os intervalos restantes são atribuídos ao grupo correspondente. Se $R R_{\text {mínimo }}+$ $(i-1) \cdot \frac{\left(R R_{\text {máximo }}-R R_{\text {mínimo })}\right.}{16} \leq R R[n]<R R_{\text {mínimo }}+(i) \cdot \frac{\left(R R_{\text {máximo }}-R R_{\text {mínimo }}\right)}{16}$, então $\mathrm{RR}[\mathrm{n}]$ pertence a classe $g_{i}$, para $\mathrm{i}=1,2, \ldots, 16$.

A entropia de Shannon é dada pelo valor negativo do número de elementos $n_{i}$ no grupo $g_{i}$, multiplicado pelo seu logaritmo natural e normalizado por um fator $\ln (1 / 16)$, da forma

$$
\text { ShanEn }=-\sum_{l=1}^{16} n_{i} \frac{\ln \left(n_{i}\right)}{\ln (1 / 16)}
$$


Figura 3: Gráfico do número dos intervalos RR em cada grupo de índice $i$ para um segmento com FA.

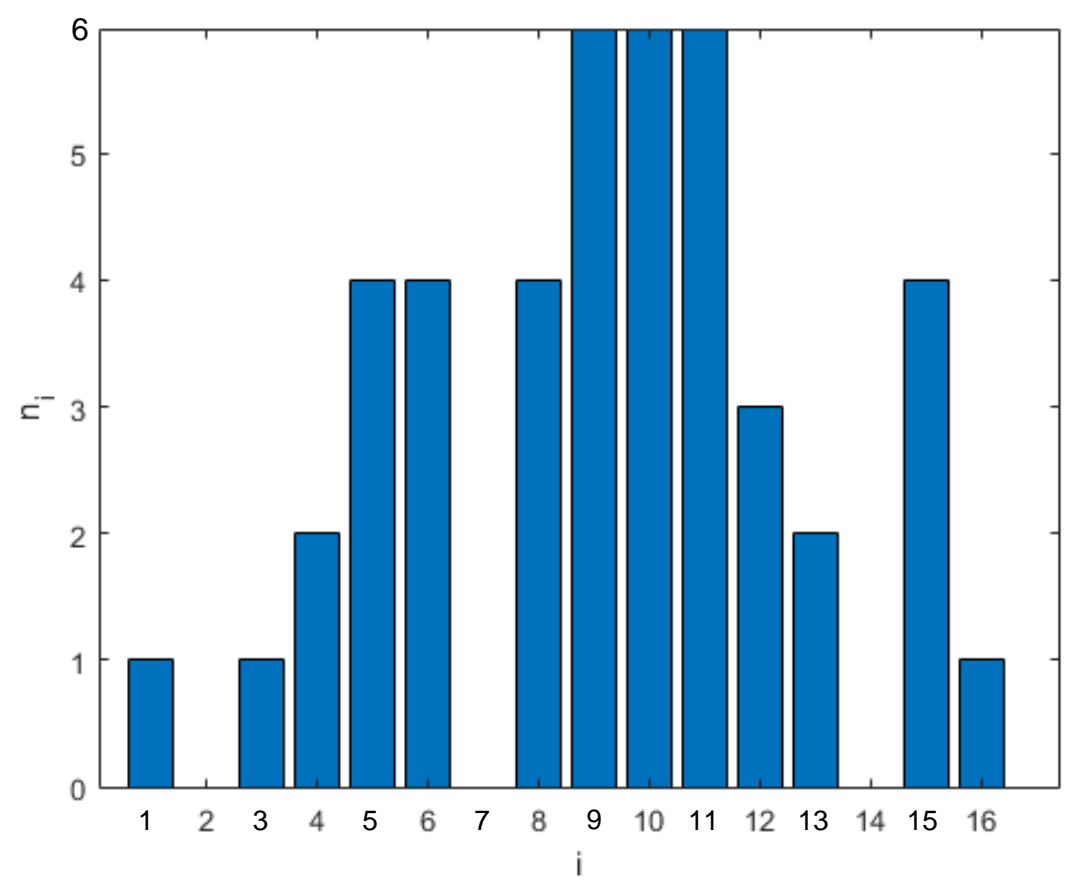

Fonte: Próprio autor.

\subsubsection{Parâmetros sugeridos por Gilani e seus colaboradores}

Dos parâmetros sugeridos por Gilani e seus colaboradores (2016), primeiramente foi analisado o valor médio dos intervalos $R R$

$$
M V=\sum_{n=1}^{60} R R[n] / N
$$

em que $N=60$ é o número de intervalos RR.

Para cada janela de 50 intervalos RR deslocando de 1 foi calculado o seu desviopadrão, dessa forma obtiveram-se 11 valores de desvio padrão, cada um associado a uma das janelas. Para a sequência de 11 desvios padrão, calculou-se a sua média

$$
\mu_{\sigma_{\mathrm{RRw}}}=\sum_{m=0}^{10} \sigma_{R R w}[m] / 11
$$


e seu desvio padrão (SDSDW)

$$
S D S D W=\sqrt{\sum_{m=0}^{10}\left(\sigma_{R R W}[m]-\mu_{\sigma_{\mathrm{RR}}}\right)^{2} / 11}
$$

em que $\sigma R R w[m]$ para $m=0,1, \ldots, 10$ é o desvio padrão da janela de 50 intervalos $R R,[R R(m+1), R R(m+2), \ldots, R R(m+50)]$.

Além do parâmetro desvio padrão da sequência de desvios padrão (SDSDW), calculou-se também o número de desvios padrão com valores superiores a 0,2, denominado contagem de janelas de desvio padrão (CSDW).

Além desses três parâmetros, Gilani e seus colaboradores (2016) também usaram dois parâmetros obtidos do gráfico de Lorenz. O gráfico de Lorenz representa os intervalos $\Delta R R[n+1] \times \Delta R R[n]$ (em que $\Delta R R[n]=R R[n]-R R[n-1]$ é a diferença dos intervalos RR consecutivos). Desse gráfico, foram extraídos dois parâmetros: o número de pontos dentro do círculo de raio 0,012 centrado na origem (Dist12) e a soma das distâncias dos pontos à origem, somente para os pontos com distância menor que 0,040 (Dist04). 
Figura 4: Gráfico de Lorenz para um segmento normal. Os pontos dentro do círculo tracejado e os dentro do círculo contínuo apresentam respectivamente distâncias menores que 0,040 e 0,012 em relação à origem.

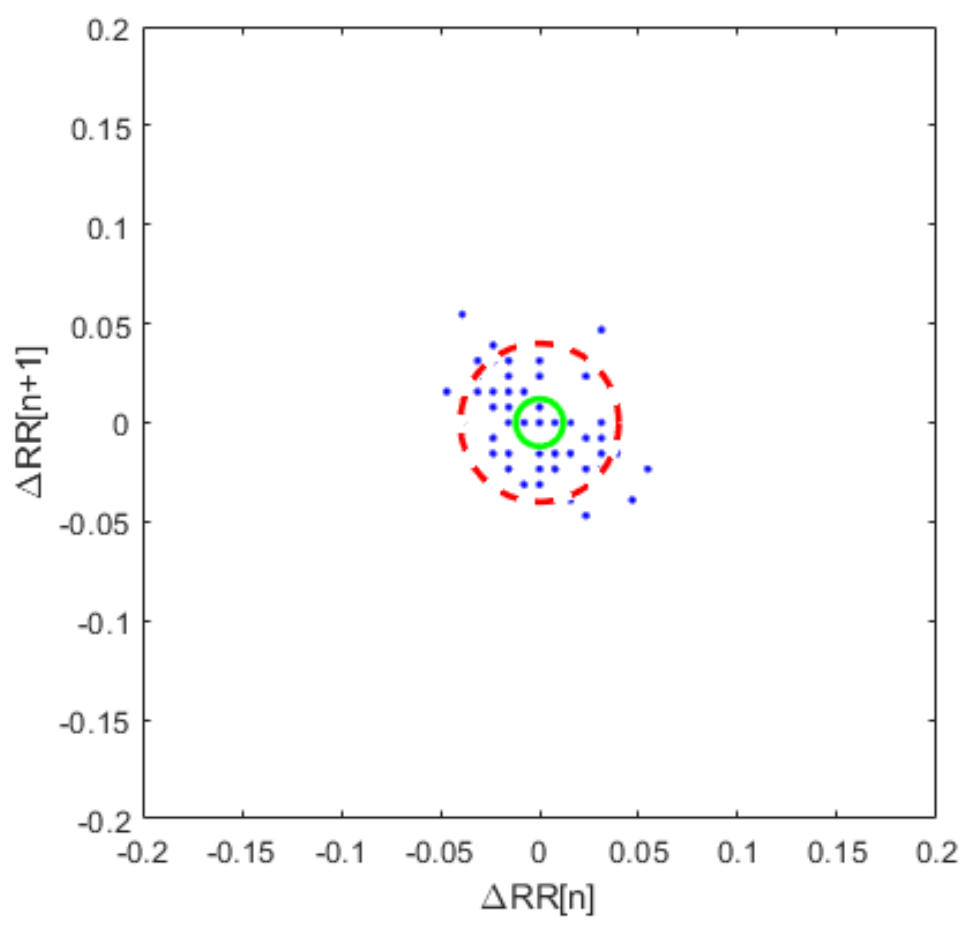

Fonte: Próprio autor.

\subsubsection{Parâmetros sugeridos por Islam e seus colaboradores}

Para o cálculo do parâmetro sugerido por Islam e seus colaboradores (2016) foi realizada a normalização dos intervalos $\mathrm{R} R$ da forma

$$
R R_{\text {norm }}[n]=0,8(R R[n]-\mu) / \mu
$$

em que $\mu$ indica a média dos $N=60$ intervalos RR. 
Figura 5: Gráfico da sequência dos intervalos $R R$ e da respectiva sequência normalizada, para um segmento com fibrilação atrial. A média está representada em linha contínua e as linhas tracejadas representam a média \pm o desvio padrão.
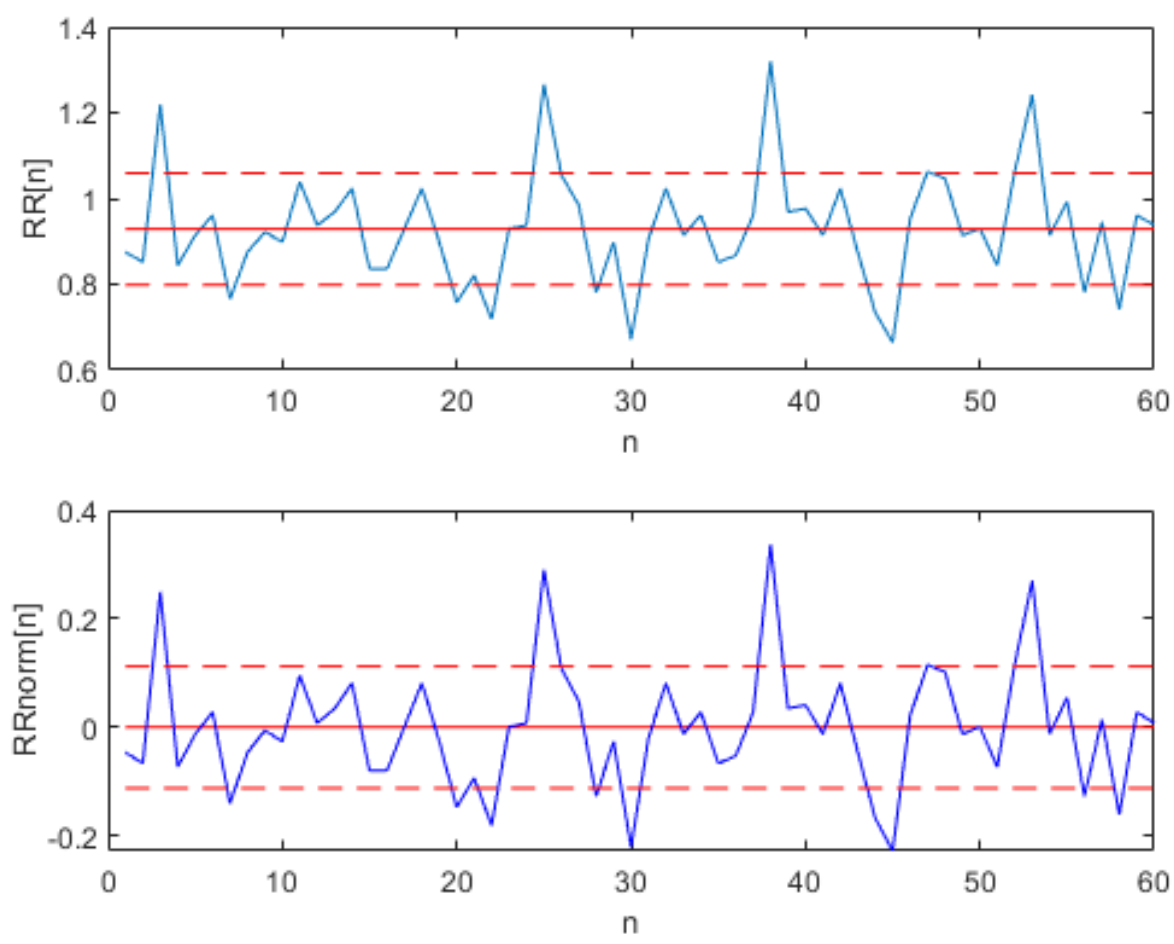

Fonte: Próprio autor.

A partir dos intervalos RR normalizados foi calculada a entropia de Shannon (ShanEn2), conforme sugerido por Dash e seus colaboradores (2009). 


\subsubsection{Parâmetros sugeridos por Kennedy e seus colaboradores}

Para o cálculo dos parâmetros sugeridos por Kennedy e seus colaboradores (2016) também foi realizada a filtragem dos intervalos RR por meio de um filtro do tipo mediana de tamanho 3 , em que

$$
R R_{\text {mediana }[n]}=\text { mediana }(R R[n], R R[n+1], R R[n+2]) .
$$

Para os intervalos filtrados $R R_{\text {mediana}}[\mathrm{n}]$, calculou-se o parâmetro coeficiente de variação

$$
C V=\sigma / \mu,
$$

em que $\sigma$ indica o desvio padrão e $\mu$ indica a média dos intervalos filtrados $R R_{\text {mediana }}[n]$. Além do coeficiente de variação, forma considerados os parâmetros raiz da média quadrática $\left(\mathrm{RMSSD}_{2}\right)$

$$
\operatorname{RMSSD}_{2}=\sqrt{\sum_{n=1}^{N-3} \frac{\left(\mathrm{RR}_{\text {mediana }}[\mathrm{n}+1]-\mathrm{RR}_{\text {mediana }}[\mathrm{n}]\right)^{2}}{N-3}}
$$

e mediana do desvio absoluto (MAD)

$$
M A D=\text { mediana }\left(\left|R R_{\text {mediana }}[n]-\operatorname{mediana}\left(R_{\text {mediana }}[n]\right)\right|\right),
$$

em que | | indica o valor absoluto e $N=60$ intervalos RR. 
Figura 6: Sequência dos intervalos RR da primeira figura 5 filtrada por um filtro do tipo mediana de tamanho 3 e dos seus valores absolutos subtraídos pela mediana. $\mathrm{Na}$ figura superior, a média das medianas está representada em linha contínua, a média \pm desvio padrão em linha tracejada. Em ambas as figuras, a respectiva mediana está representada por quadrados.
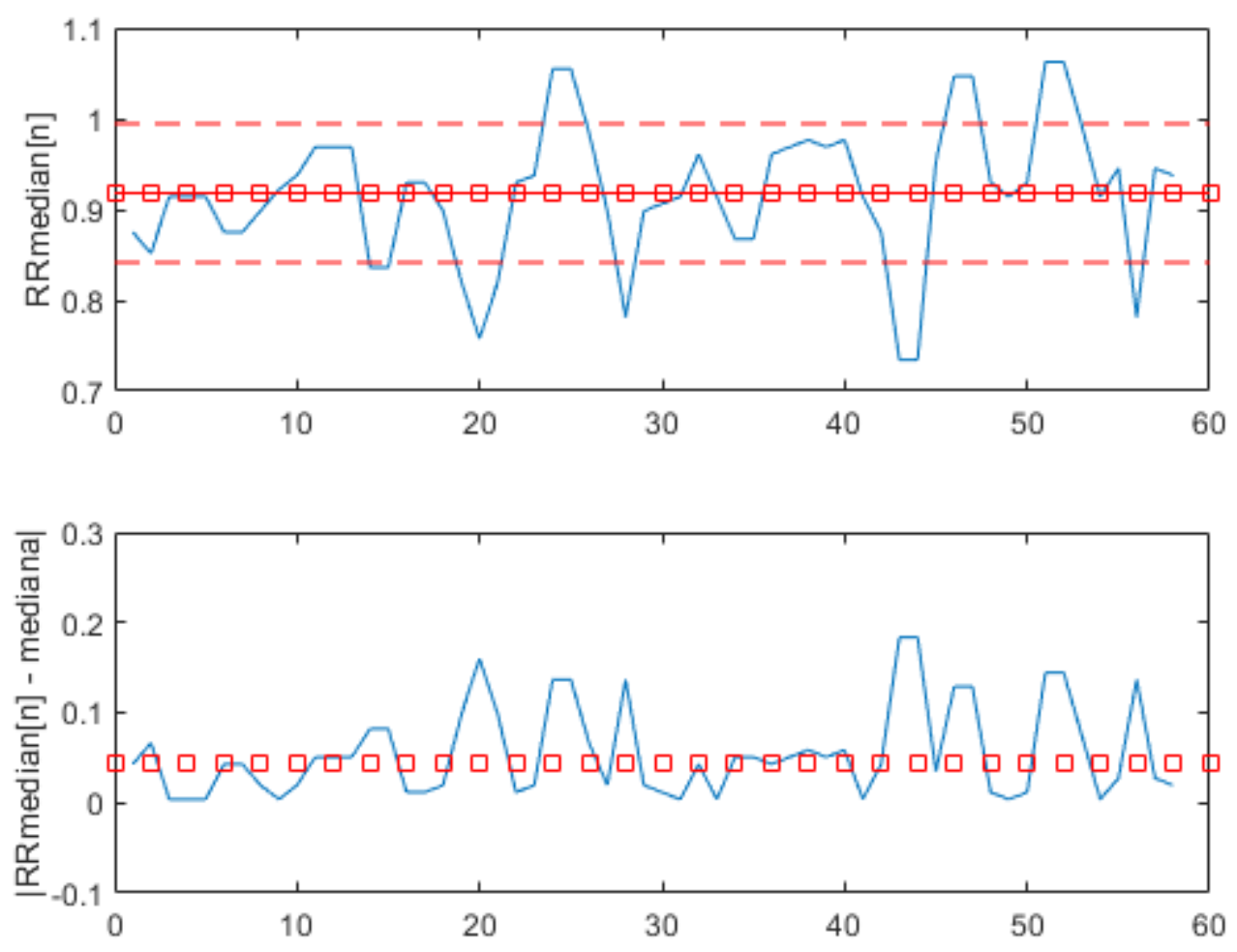

Fonte: Próprio autor.

Também foram usados parâmetros derivados da entropia amostral. Inicialmente, para o cálculo das entropias, foram obtidas variáveis auxiliares como o máximo valor absoluto da diferença escalar entre vetores de dois elementos

$\mathrm{V}_{2}[\mathrm{j}, \mathrm{k}]=\max \left\{\left|\mathrm{R} R_{\text {mediana}}[\mathrm{k}]-\mathrm{RR}_{\text {mediana }}[\mathrm{j}]\right|,|\mathrm{R} \mathrm{Rmediana}[\mathrm{k}+1]-\mathrm{RRmediana}[\mathrm{j}+1]|\right\}$,

para $1 \leq \mathrm{j}, \mathrm{k} \leq \mathrm{N}-3$, em que $N=60$ intervalos $\mathrm{RR}$. 
Figura 7: Demonstração da obtenção do valor $V_{2}$ para $k=13$. a) Sequência dos intervalos RRmediana da primeira figura 6 . b) $R R_{\text {mediana }}[13,14]$ se deslocando de $j=1$ até $j=\mathrm{N}-3$. c) $\mathrm{O}$ valor máximo das diferenças da sequência em a) pelo b).

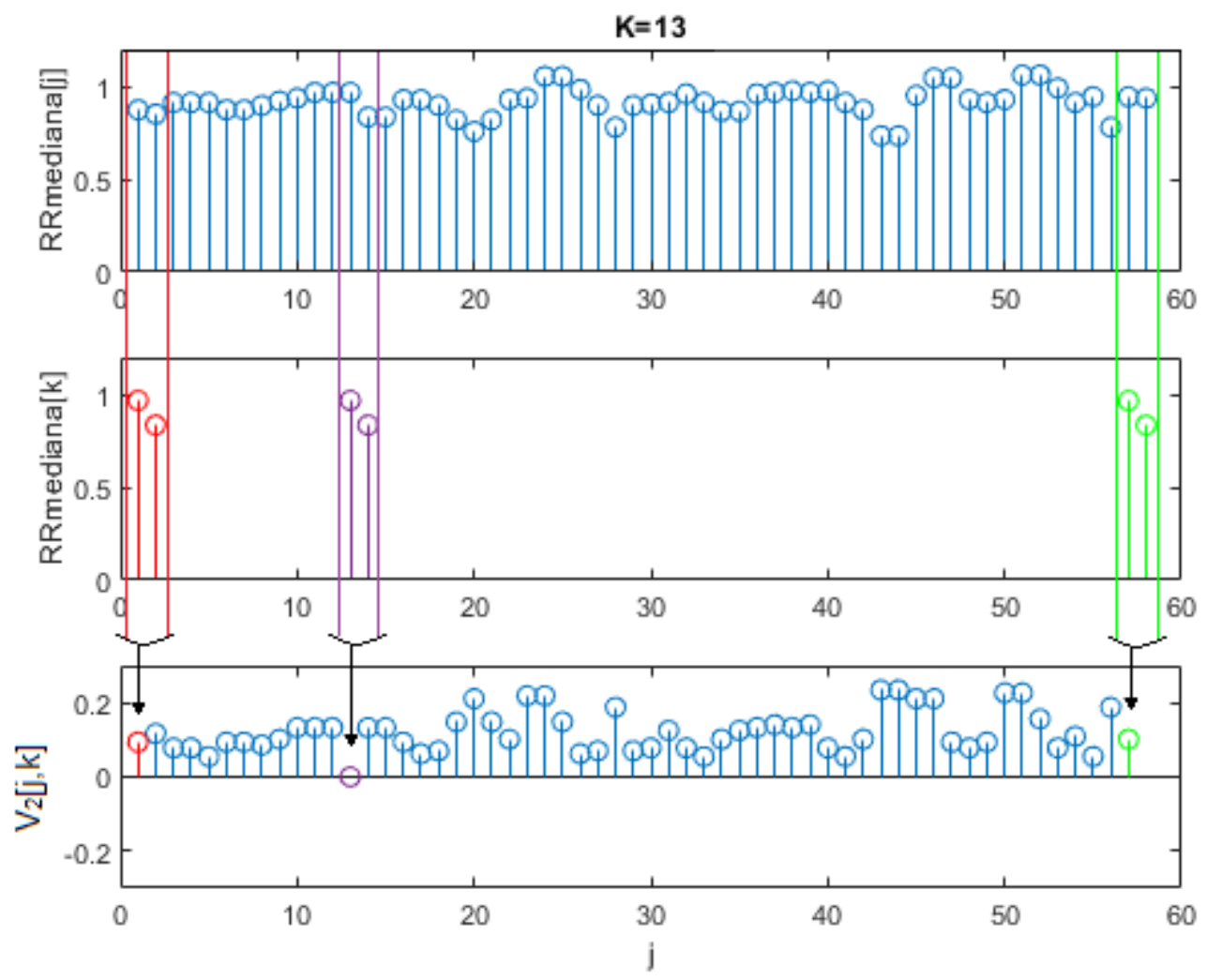

Fonte: Próprio autor.

Semelhantemente o máximo valor absoluto da diferença para vetores de três elementos foi calculado da forma

$$
\mathrm{V} 3[\mathrm{j}, \mathrm{k}]=\max \left\{\begin{array}{l}
|\mathrm{RRmediana}[\mathrm{k}]-\mathrm{RRmediana}[\mathrm{j}]|, \ldots \\
|\mathrm{RRmediana}[\mathrm{k}+1]-\mathrm{RRmediana}[\mathrm{j}+1]|, \ldots \\
\mid \text { RRmediana }[\mathrm{k}+2]-\mathrm{RRmediana}[\mathrm{j}+2] \mid
\end{array}\right\},
$$

para $1 \leq \mathrm{j}, \mathrm{k} \leq \mathrm{N}-4$, em que $N=60$ intervalos $R R$.

Em seguida, calculou-se $\mathrm{N}_{2}[\mathrm{k}]$, o número de índices $j$ para os quais $\mathrm{V}_{2}[j, k]$ é menor ou igual ao limiar, para $\mathrm{k}=1,2, \ldots, \mathrm{N}-3$, dividido por $\mathrm{N}-3$. Semelhantemente, $\mathrm{N}_{3}[\mathrm{k}]$ indica o número de índices $j$, para os quais $\mathrm{V}_{3}[\mathrm{j}, \mathrm{k}]$ é menor ou igual ao limiar de tolerância, dividido por N-4. O limiar de tolerância foi definido como $r=0,05$. 
A partir dessas variáveis auxiliares, calcularam-se os parâmetros para a classificação, que foram a entropia amostral

$$
\operatorname{SampEn}=-\ln \left(\frac{\text { média }\left(N_{3}[k]\right)}{\operatorname{média}\left(N_{2}[k]\right)}\right)
$$

e o coeficiente da entropia amostral

$$
\text { COSEn }=\operatorname{SampEn}-\ln (2 r)-\ln (\mu),
$$

em que o limiar $r=0,05$.

\subsubsection{Parâmetros sugeridos por Logan e Healey}

Para o cálculo do parâmetro sugerido por Logan e Healey (2005) também foi realizada a filtragem dos intervalos $\mathrm{RR}$, da forma iterativa

$$
R R_{\text {iterativa }}[n+1]=0,75 R R_{\text {iterativa }}[n]+0,25 R R[n+1]
$$

em que $R R_{\text {iterativa }}[1]=R R[1]$

Posteriormente, os valores filtrados foram multiplicados por 100 e calculou-se o parâmetro variância (VAR) que foi usado na classificação:

$$
V A R=\sum_{n=1}^{N}\left(R R_{\text {iterativa }}[n]-\mu\right)^{2} / N
$$

Figura 8: Sequência dos intervalos RR da primeira figura 5 filtrada por um filtro iterativo. A média está representada pela linha contínua e a média \pm desvio padrão na linha tracejada. A variância obtida foi: 0,0019.

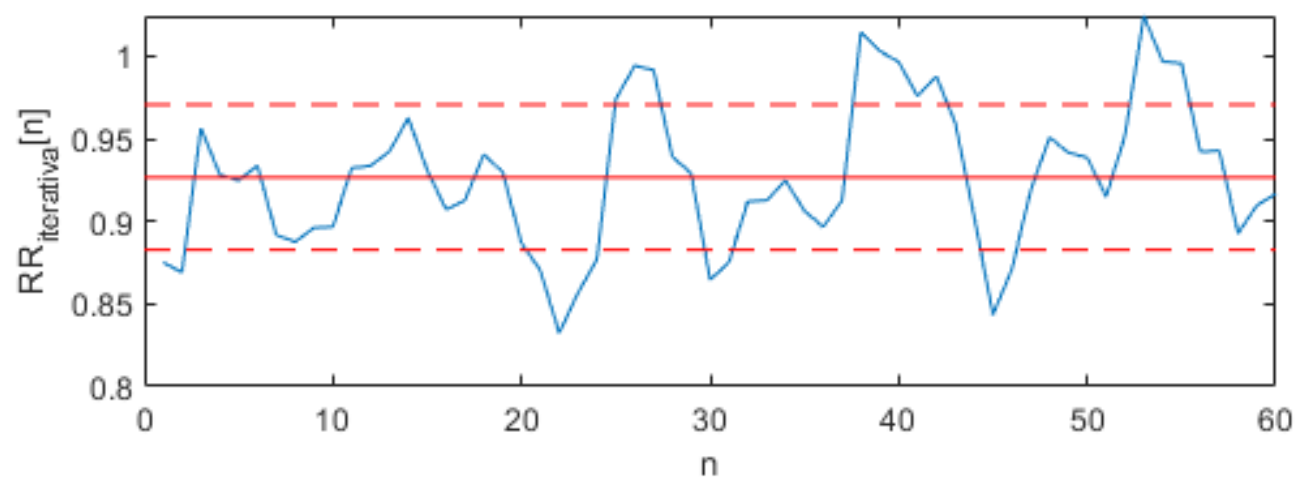

Fonte: Próprio autor. 


\subsubsection{Parâmetros sugeridos por Mabrouki e seus colaboradores}

Mabrouki e seus colaboradores (2016) sugeriram o uso de um parâmetro obtido a partir do gráfico de Poincaré.

Definem-se os vetores $x=[R R[1], R R[2], \ldots R R[N-1]]$ e $y=[R R[2], R R[3], \ldots, R R[N]]$ cujos elementos formam os pontos $R R[n+1]$ versus $R R[n]$ do gráfico de Poincaré. $O$ parâmetro sugerido por Mabrouki e seus colaboradores (2016) foi a área da elipse

$$
\mathrm{SDXY}=\pi \mathrm{SD} 1 \mathrm{SD} 2
$$

em que SD1 é o desvio padrão das distâncias $(x-y) / \sqrt{2}$ entre os pontos e a reta diagonal, e SD2 é o desvio padrão das distâncias entre os pontos e a reta perpendicular à diagonal $(x+y) / \sqrt{2}$.

Figura 9: Gráfico de Poincaré, reta diagonal e elipse com eixos menor (SD1) e maior (SD2).

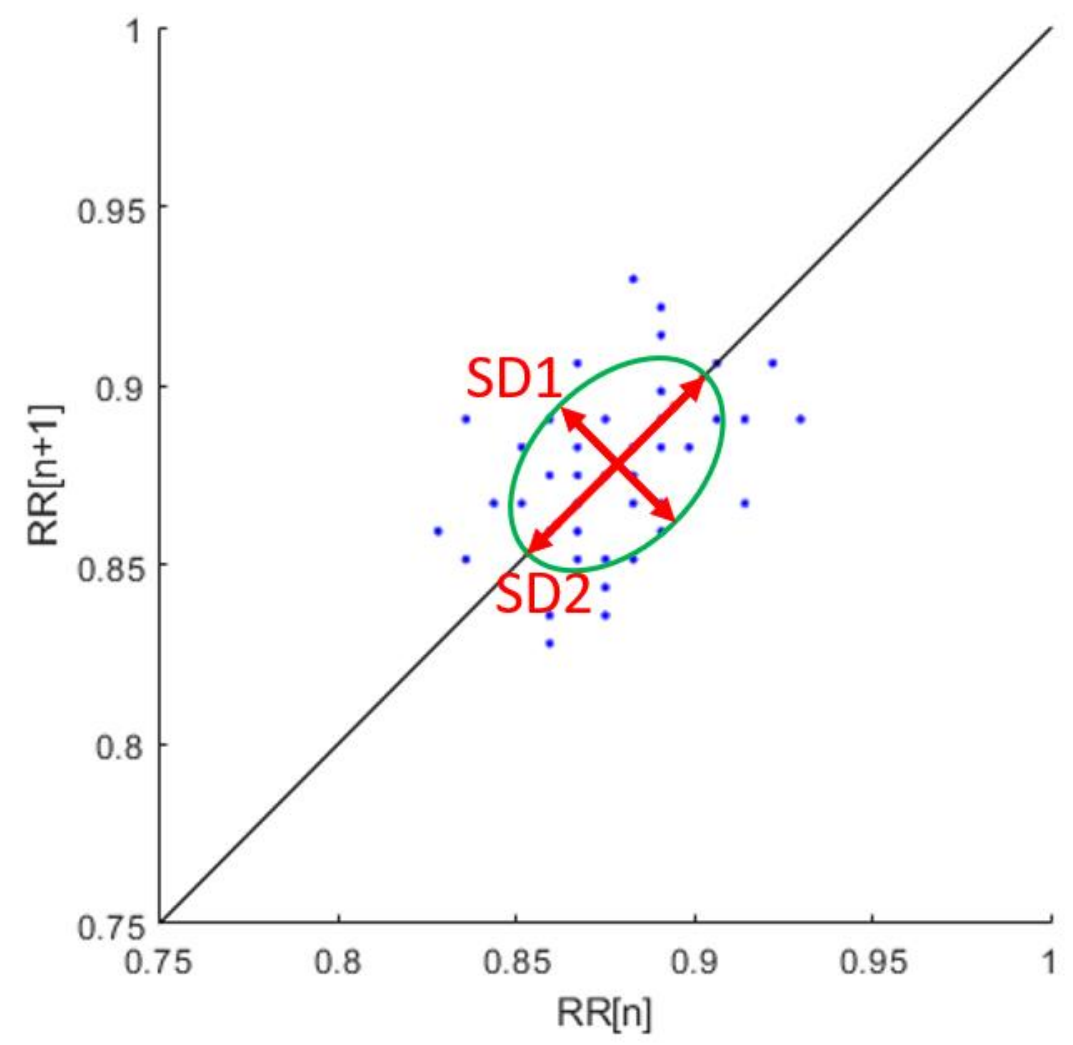

Fonte: Próprio autor. 


\subsubsection{Parâmetros sugeridos por Marsili e seus colaboradores}

Para o cálculo do parâmetro sugerido por Marsili e seus colaboradores (2016) foi realizada a discretização dos valores dos intervalos $R R$, multiplicando os valores por 50 e aproximando o valor para o inteiro mais próximo. Valores superiores a 63 foram saturados em 63.

Os valores discretizados foram filtrados, gerando a nova sequência

$$
R R 2[n]=64 R_{\text {discretizado }}[\mathrm{n}]+\mathrm{RR}_{\text {discretizado }}[\mathrm{n}+1]
$$

Figura 10: Gráfico dos valores discretizados filtrados da sequência mostrada na figura 5.

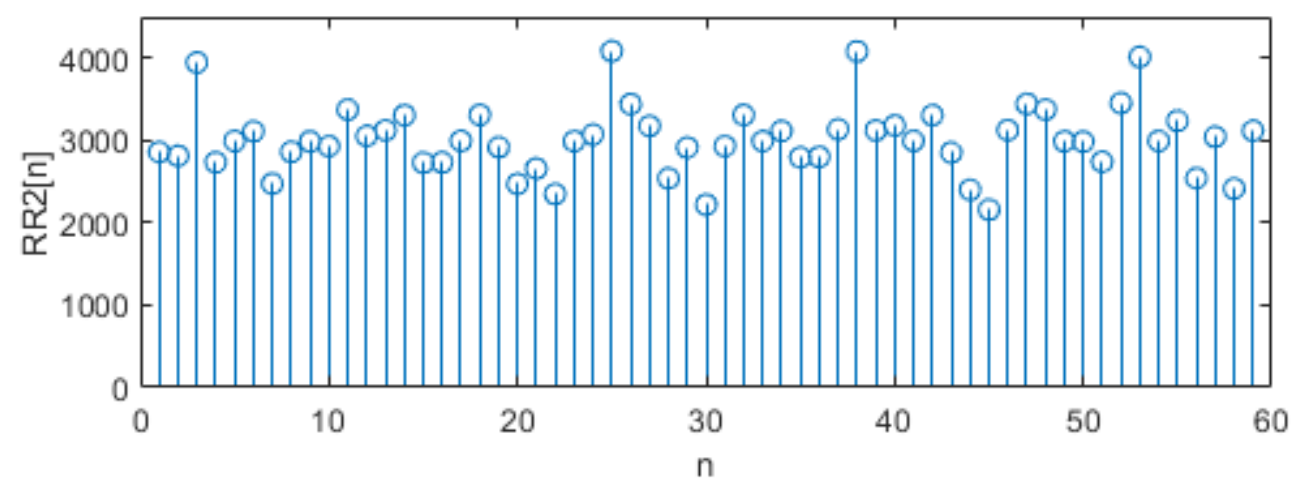

Fonte: Próprio autor.

Posteriormente os intervalos RR2 de valores iguais são agrupados. E o parâmetro entropia de Shannon foi calculado da forma:

$$
\operatorname{ShanEn}_{3}=-\sum_{i=1}^{M} \frac{n_{i} \log _{2}\left(n_{i}\right)}{59 \log _{2}(59)},
$$

em que $\mathrm{M}$ é o número de grupos formados e $\mathrm{n}_{\mathrm{i}}$ é o número de intervalos RR2 no grupo de índice $i$. 


\subsubsection{Parâmetros sugeridos por Meza}

Os parâmetros sugeridos por Meza (2015) foram obtidos a partir dos denominados batimentos irregulares. Um intervalo $R R$ é caracterizado como irregular caso a diferença absoluta do seu valor em relação a mediana for maior que 0,45 da média dos intervalos.

Os parâmetros analisados foram o número de intervalos irregulares divido pela quantidade total de intervalos (QIr), a média (MVIr), mediana (Mdlr) e o desvio padrão (DPIr) dos intervalos irregulares.

Figura 11: Gráfico superior representa as diferenças absolutas em relação a mediana. A linha com traços e pontos é o limiar de comparação e corresponde à média dos intervalos RR originais multiplicada por 0,45 . O gráfico inferior mostra os intervalos considerados irregulares, a respectiva média em traço contínuo, mediana em pontilhado e média \pm desvio padrão em tracejado. Ambos os gráficos foram obtidos a partir do sinal original mostrado na figura 5
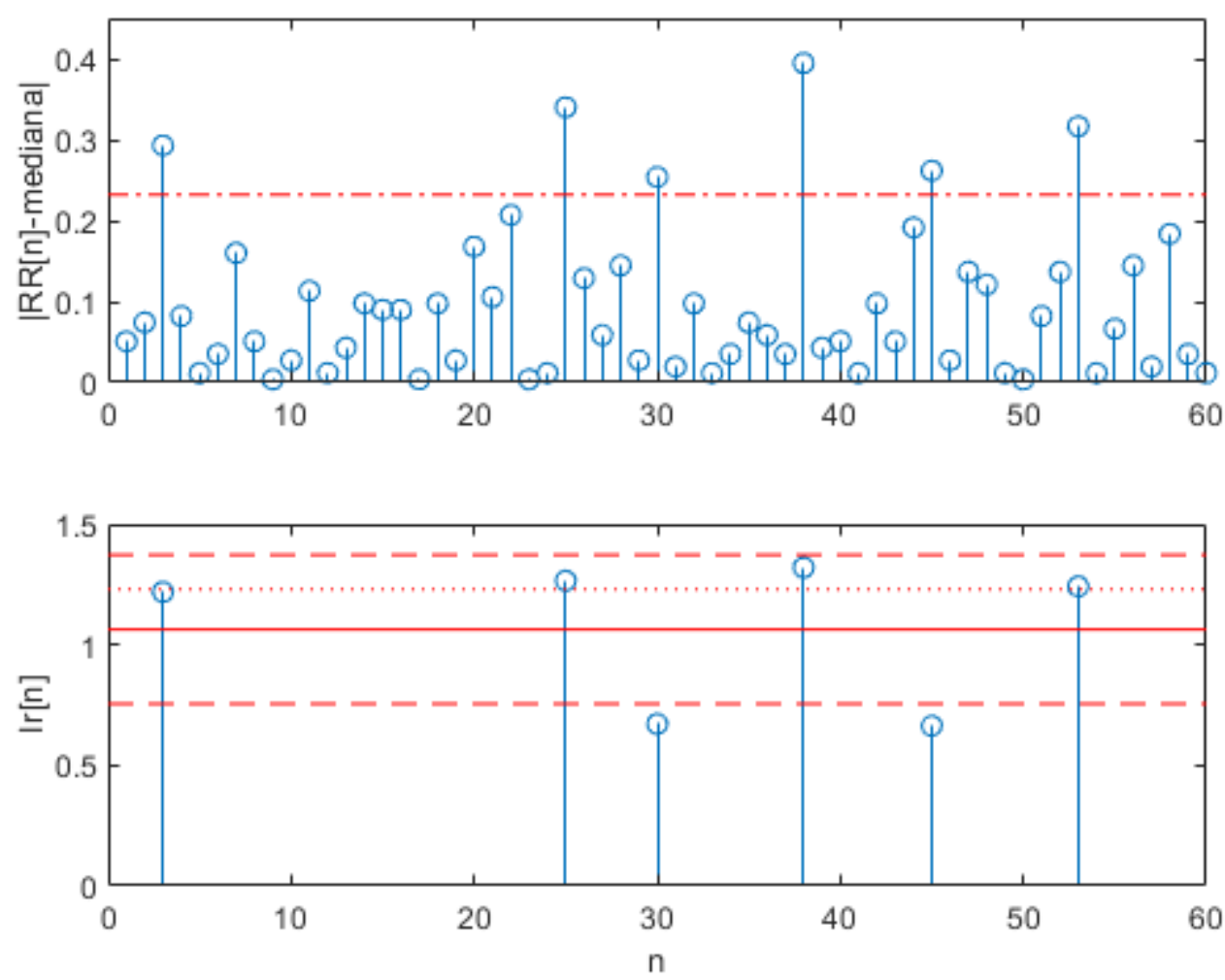

Fonte: Próprio autor. 


\subsubsection{Parâmetros sugeridos por Mohebbi e Ghassemian}

Dos parâmetros sugeridos por Mohebbi e Ghassemian (2012), foram analisados a entropia amostral, e os parâmetros obtidos a partir do gráfico de Poincaré.

A entropia amostral (SampEn2) obtida por Mohebbi e Ghassemian é a mesma obtida por Kennedy e seus colaboradores (2016), mas diretamente aplicada aos intervalos RR e não aos intervalos após a filtragem. O limiar de tolerância utilizado foi de $20 \%$ do desvio-padrão dos intervalos RR

$$
r_{i}=0,20 \sqrt{\sum_{n=1}^{N}\left(R R[n]-\sum_{n=1}^{N} R R[n] / N\right)^{2} / N},
$$

em que $N$ é igual a 60 intervalos $R R$.

Para o gráfico de Poincaré RR [n+1] versus RR [n], obtiveram-se as distâncias I1[n] entre os pontos ( $R R[n+1], R R[n])$ e a reta com coeficiente angular unitário que passa pela origem. Na classificação, foi usado o desvio padrão das distâncias dos pontos à reta que passa pela origem

$$
P C S D 1=\sqrt{\sum_{n=1}^{N-1}\left(l 1[n]-\sum_{n=1}^{N-1} l 1[n] / N-1\right)^{2} /(N-1)},
$$

em que $l 1[n]=|R R[n+1]-R R[n]| / \sqrt{2}$. 
Também foram calculadas as distâncias $I 2[n]$ dos pontos $(R R[n+1], R R[n])$ até a reta de coeficiente angular -1 , que passa pelo ponto $(-2 \mu, 0)$ ou $(0,-2 \mu)$, em que $\mu$ é a média dos intervalos RR. Utilizou-se na classificação o desvio padrão das distâncias dos pontos à reta que passa pela média dos intervalos RR é dada por

$$
\operatorname{PCSD} 2=\sqrt{\sum_{n=1}^{N-1}\left(l 2[n]-\sum_{n=1}^{N-1} l 2[n] / N-1\right)^{2} /(N-1)},
$$

em que $l 2[n]=|R R[n+1]+R R[n]-2 \mu| / \sqrt{2}$.

Figura 12: Exemplo de um gráfico de Poincaré com a reta diagonal com coeficiente angular unitário em linha contínua e a reta tracejada de coeficiente angular -1 que passa pelo ponto $(-2 \mu, 0)$ ou $(0,-2 \mu)$.

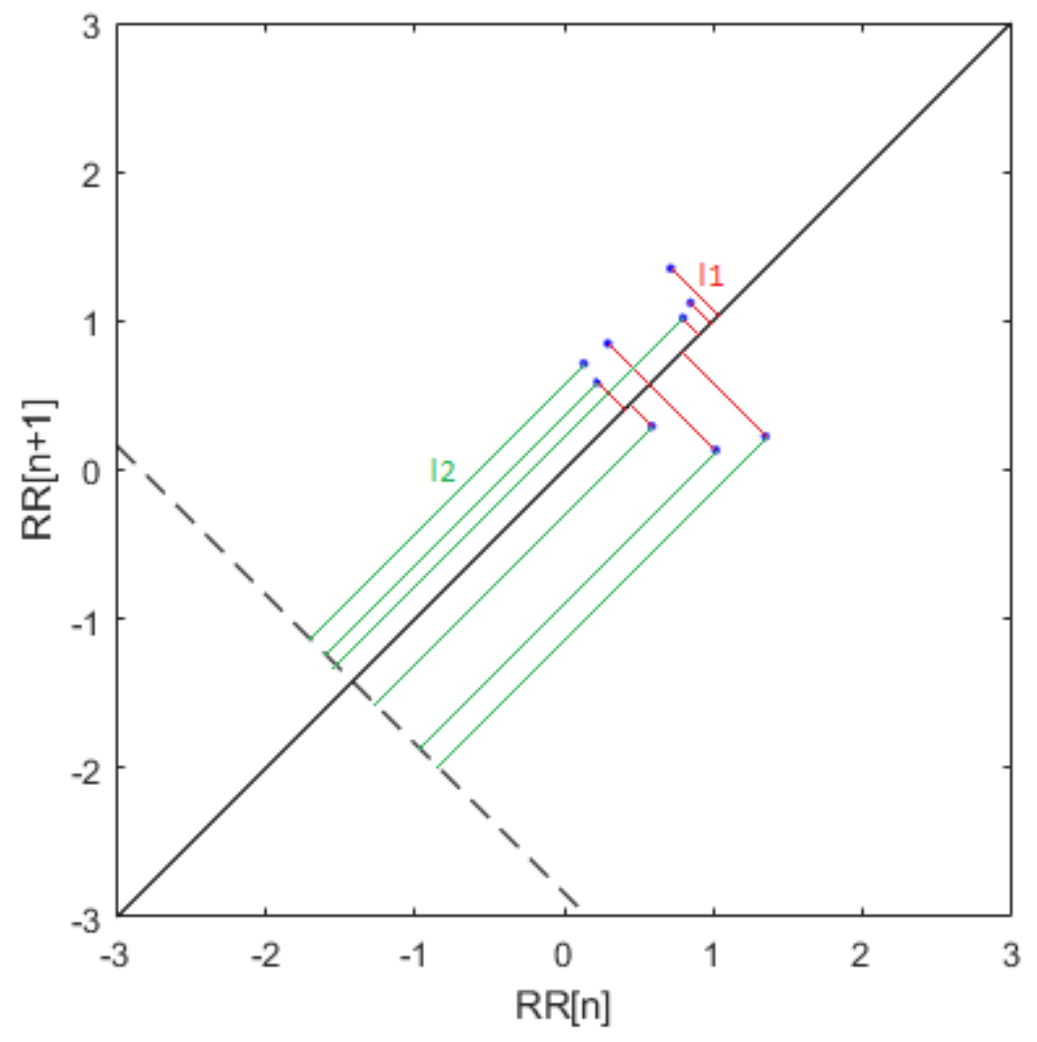

Fonte: Próprio autor. 


\subsubsection{Parâmetros sugeridos por Nuryani e seus colaboradores}

Nuryani e seus colabores (2015) usaram as diferenças $(\Delta R R)$ entre intervalos $R R$ consecutivos.

Os parâmetros sugeridos por eles foram a média (MV $\Delta R R$ ) e o desvio padrão (SDSD) dessas diferenças $\Delta R R$.

\subsubsection{Parâmetros sugeridos por Slocum e seus colaboradores}

Slocum e seus colaboradores (1992) centralizaram os segmentos de ECG e preencheram o início e fim dos segmentos com zeros até que tivesse comprimento de 4096 amostras. Em seguida, calcularam a transformada discreta de Fourier (DFT, do inglês discrete Fourier transform)

$$
\operatorname{DFT}[k]=\sum_{m=1}^{M} E C G[n] \cdot \exp ((-2 \pi j / M)(m-1)(k))
$$

para $k=0,1,2, \ldots, M$; em que $M=4096$ e $j=\sqrt{(-1)}$. Em seguida, sugeriram como parâmetro da classificação a razão entre a soma dos módulos dos componentes da DFT na faixa de 5 a $9 \mathrm{~Hz}$ e a soma dos módulos na faixa de 1 a $57 \mathrm{~Hz}$ (REB).

\subsubsection{Parâmetros sugeridos por Trardi e seus colaboradores}

Os parâmetros sugeridos por Trardi e seus colaboradores (2018) não foram computados somente para os segmentos contendo 60 intervalos RR consecutivos representados por $R R[n]$, para $n=1,2, \ldots, 60$. Também foram obtidos para o primeiro, segundo e terceiro sinais diferenciados absolutos dos intervalos RR. Os sinais diferenciados de ordem $i$ são dados por

$$
R R[i, n]=|R R[i-1, n+1]-R R[i-1, n]|,
$$

para $n=1,2, \ldots, N$; em que $N=60-i$ e $\operatorname{RR}[0, n]=R R[n]$ 
Para a classificação, obtiveram-se diversos parâmetros. Para cada segmento foram calculados o valor médio

$$
\mathrm{MV}_{i}=\sum_{n=1}^{N} R R[i, n] / N
$$

a raiz da média quadrática

$$
\mathrm{RMS}_{i}=\sqrt{\sum_{n=1}^{N} R R^{2}[i, n] / N}
$$

o coeficiente de curtose

$$
\mathrm{KC}_{i}=\frac{\sum_{n=1}^{N}\left(R R[i, n]-M V_{i}\right)^{4} / N}{\left.\sum_{n=1}^{N}\left(\left(R R[i, n]-M V_{i}\right)^{2}\right) / N\right)^{2}}
$$

e o coeficiente de assimetria

$$
\mathrm{SC}_{i}=\frac{\sum_{n=1}^{N}\left(R R[i, n]-M V_{i}\right)^{3} / N}{\left.\sum_{n=1}^{N}\left(\left(R R[i, n]-M V_{i}\right)^{2}\right) / N\right)^{3 / 2}}
$$

Dividindo os intervalos RR em três sequências do mesmo tamanho, foram calculadas a mediana de cada sequência subtraída pela sua média

$$
\begin{aligned}
& d_{0}=\text { mediana }(R R[i, m])-\text { média }(R R[i, m]), \\
& d_{1}=\text { mediana }(R R[i, 20+m])-\text { média }(R R[i, 20+m]), \\
& d_{2}=\text { mediana }(R R[i, 40+m])-\text { média }(R R[i, 40+m]),
\end{aligned}
$$

para $\mathrm{k}=0,1,2$ e $\mathrm{m}=1,2, \ldots 20$. O parâmetro usado na classificação foi a mediana das três medianas

$$
\operatorname{MMed}_{i}=\operatorname{mediana}\left(d_{0}, d_{1}, d_{2}\right)
$$


Para gráfico de Poincaré $R R$ [i, $n+1$ ] versus $R R$ [i, n], foi extraído o ângulo entre a reta que une a origem $(0,0)$ ao ponto $(R R[i, n+1], R R[i, n])$ e a reta que define $45^{\circ}$. $\mathrm{Na}$ classificação foi usada a média do valor absoluto dos ângulos

$$
V A I_{i}=\sum_{n=1}^{N-1}\left|\theta[i, n]-45^{\circ}\right| /(N-1),
$$

em que $\theta[i, n]=\operatorname{atan}(R R[i, n+1] / R R[i, n])$.

Também foram obtidas as distâncias dos pontos até a origem. Outro parâmetro utilizado na classificação foi o desvio padrão das distâncias

$$
V L I_{i}=\sqrt{\sum_{n=1}^{N-1}\left(l[i, n]-\sum_{n=1}^{N-1} l[i, n] /(N-1)\right)^{2} /(N-1)},
$$

em que $l[i, n]=\sqrt{R R^{2}[i, n+1]+R R^{2}[i, n]}$.

Figura 13: Exemplo de um gráfico de Poincaré com a reta que define $45^{\circ}$ em linha contínua e a marcação do ângulo entre a reta que une a origem ao ponto e a reta de $45^{\circ}$ e a marcação da distância do ponto até a origem.

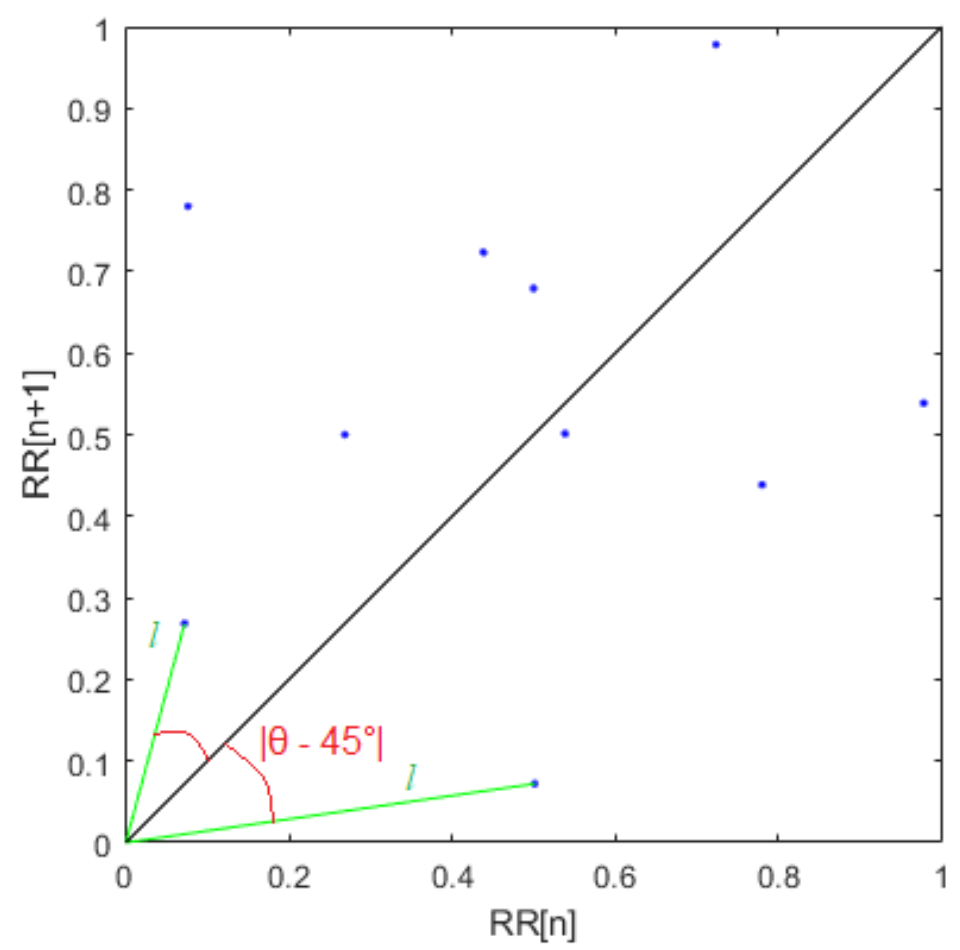

Fonte: Próprio autor. 
Os parâmetros relacionados às entropias, como a entropia amostral, a entropia aproximada e a entropia Fuzzy também foram usados na classificação. Para o cálculo das entropias foram usadas variáveis auxiliares como o máximo valor absoluto da diferença escalar entre dois vetores de dois elementos

$$
V_{i, 2}[j, k]=\max \{|R R[i, k]-R R[i, j]|,|R R[i, k+1]-R R[i, j+1]|\},
$$

para $1 \leq j, k \leq N-1-i$. Similarmente, para vetores de três elementos, foi calculado o máximo valor absoluto das diferenças

$$
V_{i, 3}[j, k]=\max \left\{\begin{array}{l}
|R R[i, k]-R R[i, j]|, \ldots \\
|R R[i, k+1]-R R[i, j+1]|, \ldots \\
|R R[i, k+2]-R R[i, j+2]|
\end{array}\right\},
$$

para $1 \leq j, k \leq N-2-i$.

O limiar de tolerância utilizado foi de $25 \%$ do desvio-padrão dos intervalos RR

$$
r_{i}=0,25 \sqrt{\sum_{n=1}^{N}\left(R R[i, n]-M V_{i}\right)^{2} / N}
$$

O número de índices $j$, para os quais $V_{i, 2}[j, k] \leq r_{i}$, dividido por $\mathrm{N}$-1-i é denominado $N_{i, 2}[k]$, para $\mathrm{k}=1,2, \ldots, \mathrm{N}-1$. Da mesma forma, $N_{i, 3}[k]$ indica o número de índices $j$, para os quais $V_{i, 3}[j, k] \leq r_{i}$, dividido por $\mathrm{N}-2-i$.

Também foram calculadas funções exponenciais de segunda ordem

$$
\Psi_{i, 2}[k]=\sum_{j=1}^{N-1}\left(\exp \left(-\left(V_{i, 2}[j, k]\right)^{2}\right) / r_{i}\right)
$$

para $\mathrm{j}=1,2, \ldots, \mathrm{N}-1$; e de terceira ordem

$$
\Psi_{i, 3}[k]=\sum_{j=1}^{N-2}\left(\exp \left(-\left(V_{i, 3}[j, k]\right)^{2}\right) / r_{i}\right)
$$

para auxiliar o cálculo das entropias. 
A entropia amostral é dada por

$$
\operatorname{SampEn}_{i}=-\ln \left(\frac{\operatorname{média}\left(N_{i, 3}[k]\right)}{\operatorname{média}\left(N_{i, 2}[k]\right)}\right) \text {. }
$$

A entropia aproximada é dada por

$$
A p E n_{i}=\text { média }\left(\ln \left(N_{i, 3}[k]\right)\right)-\text { média }\left(\ln \left(N_{i, 2}[k]\right)\right) \text {. }
$$

A entropia Fuzzy é dada por

$$
\text { FuzzyEn }_{i}=\ln \left(\frac{\text { média }\left(\Psi_{i, 3}[k]\right)}{\operatorname{média}\left(\Psi_{i, 2}[k]\right)}\right) \text {. }
$$

As três entropias são usadas para classificação. Além das entropias em si, também são usados o coeficiente da entropia amostral e a entropia amostral quadrática.

O coeficiente da entropia amostral é dado por

$$
\operatorname{COSEn} n_{i}=\operatorname{SampEn}_{i}-2 \ln \left(r_{i}\right)-\ln (\operatorname{mé} \operatorname{dia}(R R[i, n])),
$$

e a entropia amostral quadrática por

$$
Q S E_{i}=S a m p E n_{i}+2 \ln \left(2 r_{i}\right)
$$

A diferença entre intervalos consecutivos é calculada como $\Delta R R[i, n]=R R[i, n+1]-$ $R R[i, n]$. Também foram usados como parâmetros para classificação o desvio padrão das diferenças entre intervalos consecutivos

$$
S D S D_{i}=\sqrt{\sum_{n=1}^{N-1}\left(\Delta R R[i, n]-\sum_{n=1}^{N-1} \Delta R R[i, n] /(N-1)\right)^{2} /(N-1)},
$$


o desvio padrão dos valores absolutos das diferenças sobre raiz de 2

$$
S D_{i}=\sqrt{\sum_{n=1}^{N-1}\left(|\Delta R R[i, n]|-\sum_{n=1}^{N-1}|\Delta R R[i, n]| /(N-1)\right)^{2} /(2 N-2)},
$$

e o desvio padrão dos valores absolutos das diferenças sobre raiz de 2 menos o dobro da média de RR[i,n]

$$
S D 2_{i}=\sqrt{\sum_{n=1}^{N-1}\left(a u x-\sum_{n=1}^{N-1} a u x /(N-1)\right)^{2} /(N-1)},
$$

em que $a u x=|\Delta R R[i, n]| / \sqrt{2}-2 M V_{i}$.

\subsubsection{Outros parâmetros}

Além dos parâmetros listados anteriormente, também foram analisados outros parâmetros. Como a fibrilação atrial é caracterizada por batimentos cardíacos irregulares, a razão entre o valor máximo e o valor mínimo dos intervalos $R R$ pode ser maior na FA do que no ritmo normal. Por isso, na classificação, incluiu-se a razão entre o máximo e o mínimo intervalo RR

$$
M M R=\frac{\max (R R[n])}{\min (R R[n])} .
$$

A motivação para se propor o uso dessa razão foi o fato de Rizwan e seus colaboradores (2011) terem utilizado os máximos e mínimos intervalos RR para detectar a fibrilação atrial. Porém, nesta dissertação propôs-se a o uso da razão entre esses parâmetros.

Outro parâmetro adicionado à investigação dos possíveis atributos foi inspirado no trabalho de Wrobel e seus colaboradores (2018): a mediana dos desvios absolutos entre os intervalos RR e o valor médio, que é dada por

$$
M A D=\text { mediana }(|R R[n]-\mu|) .
$$


Além dos parâmetros VAI e VLI obtidos do gráfico de Poincaré, a média dos senos do ângulo entre a reta que une a origem $(0,0)$ ao ponto $(R R[n+1], R R[n])$ e a reta horizontal também foi adicionada à investigação dos possíveis parâmetros. Esse parâmetro é dado por

$$
S E N P=\frac{1}{N-1} \sum_{n=1}^{N-1}\left(\frac{|R R[n]-R R[n+1]|}{\sqrt{R R[n]^{2}+R R[n+1]^{2}}}\right)
$$

em que $\mathrm{N}$ é igual a 60 intervalos $\mathrm{RR}$.

Outro parâmetro adicionado foi inspirado no trabalho de Zhang (2018): a entropia de Shannon da transformação simbólica dinâmica. A transformação simbólica dinâmica consiste na discretização do sinal diferenciado absoluto

$$
d R R[n]=\left\{\begin{array}{ll}
0 & \text { caso } 0,0 \leq|\Delta R R[n]|<0,1 \\
1 & \text { caso } 0,1 \leq|\Delta R R[n]|<0,2 \\
2 & \text { caso } 0,2 \leq|\Delta R R[n]|<0,3 \\
3 & \text { caso } 0,3 \leq|\Delta R R[n]|<0,4 \\
4 & \text { caso } 0,4 \leq|\Delta R R[n]|<0,5 \\
5 & \text { caso } 0,5 \leq|\Delta R R[n]|
\end{array} .\right.
$$

O sinal discretizado é então filtrado pela equação de diferenças

$$
w R R[n]=d R R[n]+10 d R R[n-1]+100 d R R[n-2]+1000 d R R[n-3]
$$

para $\mathrm{n}=4,5, \ldots, \mathrm{N}-1$. 
Figura 14: Discretização do sinal diferenciado da sequência mostrada na figura 5 e o seu sinal filtrado.
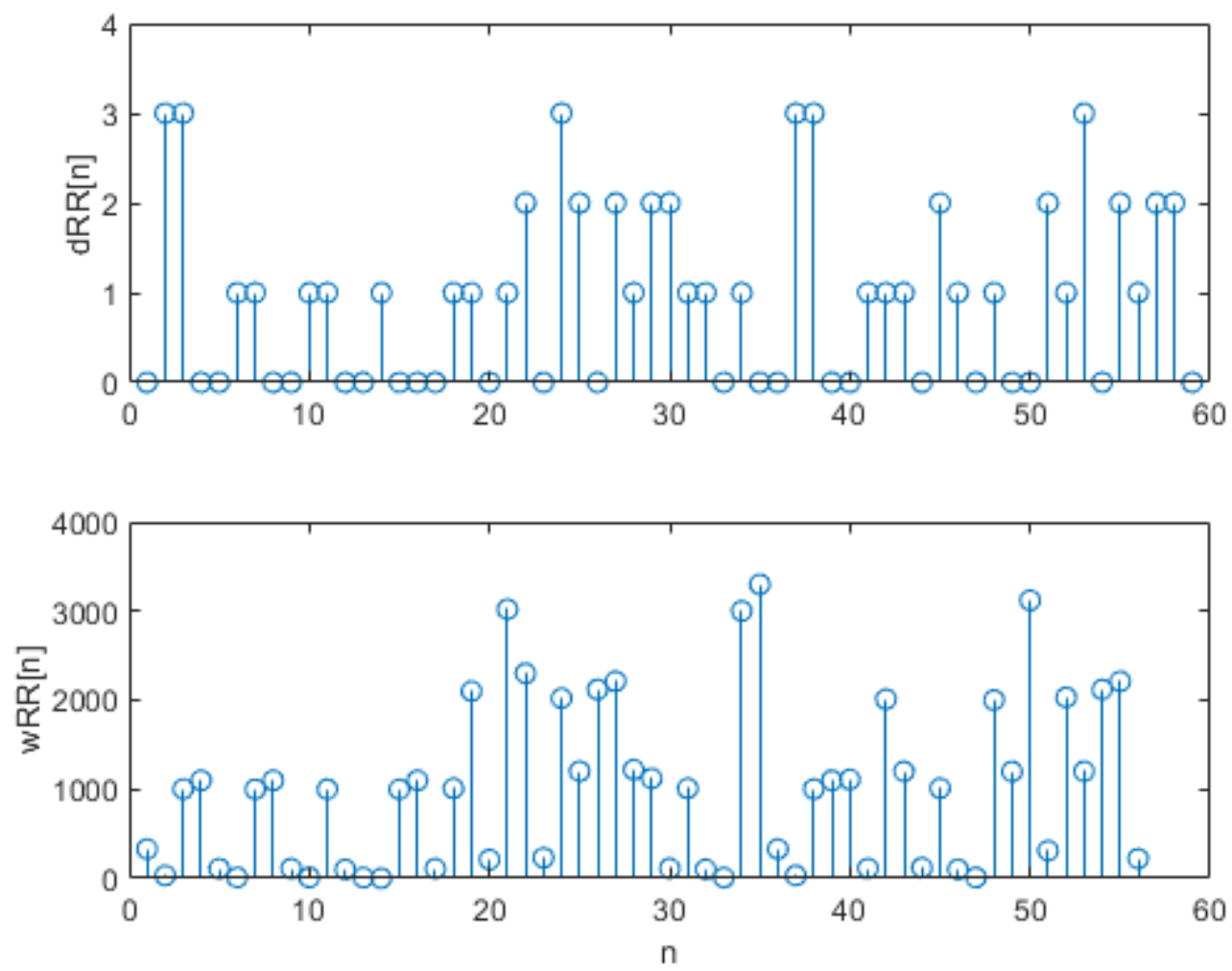

Fonte: Próprio autor.

Posteriormente os valores iguais desse sinal filtrado $w R R[n]$ são agrupados. Para esse sinal filtrado, a entropia de Shannon é dada por

$$
\text { ShanEn }=-\sum_{i=1}^{M} n_{i} \log _{2} n_{i},
$$

em que $n_{i}$ é o número de valores wRR[n] no grupo de índice $i$ e $M$ é o número de grupos.

Também foi realizado cálculo das entropias amostrais, de Fuzzy e coeficiente da entropia amostral com o limiar de tolerância otimizado. O valor encontrado do limiar ótimo para a entropia amostral e o coeficiente de entropia amostral foi $r=0,05$. E o valor de limiar ótimo para entropia de Fuzzy encontrado foi de $2 \%$ do desvio-padrão dos intervalos RR. 
Quando os batimentos são regulares, a variação em torno da média é pequena. O que seria representada por um componente DC muito maior em relação aos outros componentes espectrais. Na fibrilação atrial, ocorreriam batimentos em frequência irregular. Essa irregularidade levaria a um aumento dos componentes espectrais, exceto o DC. Por isso, outro parâmetro adicionado à investigação foi a soma dos módulos dos componentes espectrais de Fourier (exceto o nível DC) divido pelo nível DC.

Para obter os componentes espectrais, foi usada a transformada discreta de Fourier dos intervalos RR, que é dada por

$$
D F T[k]=\sum_{n=1}^{N} R R[n] \cdot \exp ((-2 \pi j / M)(m-1)(k)),
$$

em que $\mathrm{k}=0,1,2, \ldots, \mathrm{N}-1$ e $j=\sqrt{(-1)}$. A soma dos módulos dos componentes (exceto o nível DC), dividida pelo nível DC pode ser obtida por

$$
F C=\sum_{k=1}^{N-1}|D F T(k)| / D F T(0) .
$$

Figura 15: Módulo da DFT da sequência mostrada na figura 5

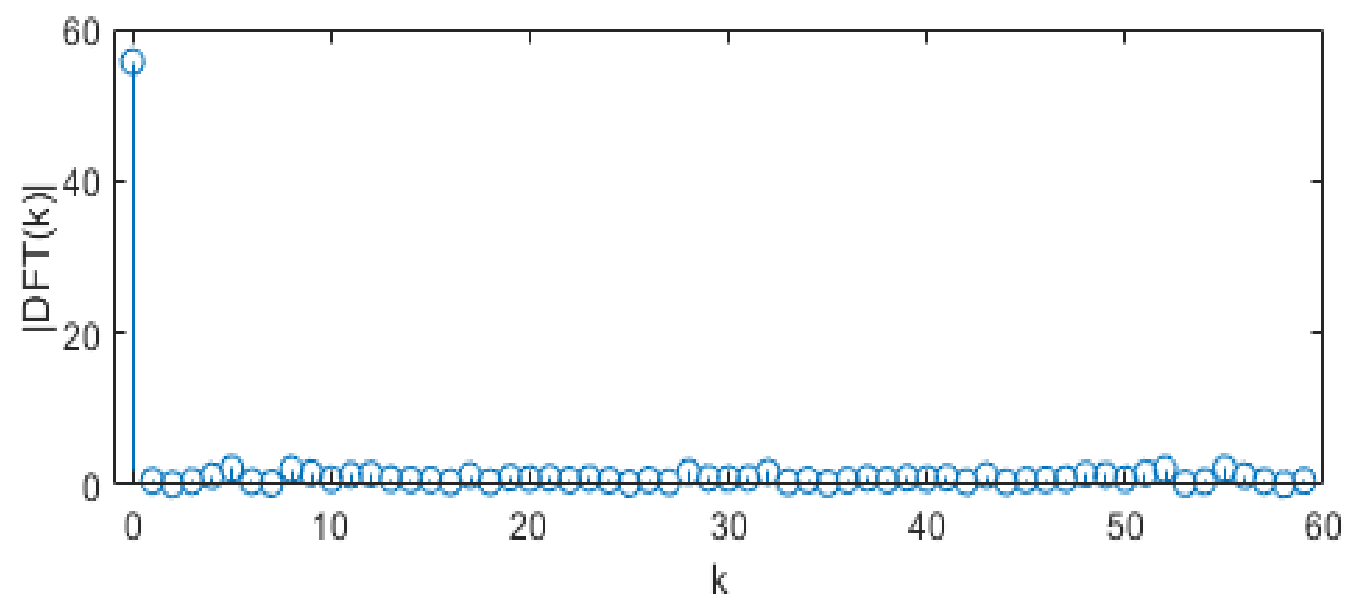

Fonte: Próprio autor. 


\subsubsection{Imagens de Poincaré}

Para a entrada da rede neural convolucional foram geradas as imagens formadas pelas retas que ligam a origem até aos pontos do gráfico de Poincaré. Para cada ponto da reta, soma-se 1 na sua posição. Dessa forma as regiões onde há maior quantidade de pontos e retas seriam representadas por tons de cinza mais claros e as regiões com menor quantidade, por cinzas mais escuros.

Como a frequência de amostragem para o banco de dados Long-Term Atrial Fibrillation é de 128 amostras por segundo e foram desconsiderados segmentos que contém intervalos RR maiores do que 2 segundos, os possíveis valores para os intervalos $R R$ variariam de 0 a 256. Por isso, a menor imagem sem perda de informação seria do tamanho 256×256.

A figura 16 mostra um exemplo de imagens de Poincaré de um segmento com FA e outro de um segmento normal. Em segmentos normais, não há uma grande variação dos intervalos RR, resultando em poucas retas com tons de cinza mais claros. Enquanto nos segmentos com FA há uma quantidade maior de retas com tons de cinza mais escuros.

Figura 16: Exemplos de imagens de Poincaré de um segmento com FA à esquerda $e$ de um segmento normal à direita.

FA

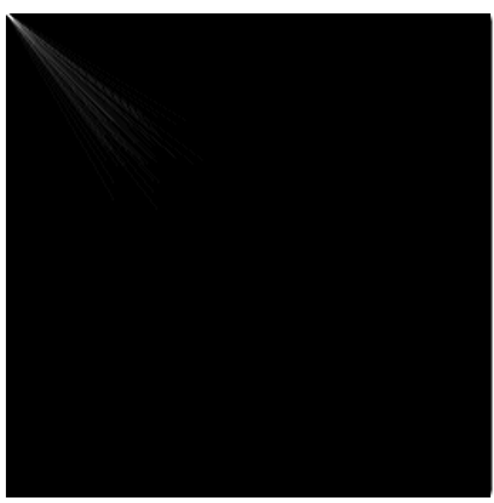

Normal

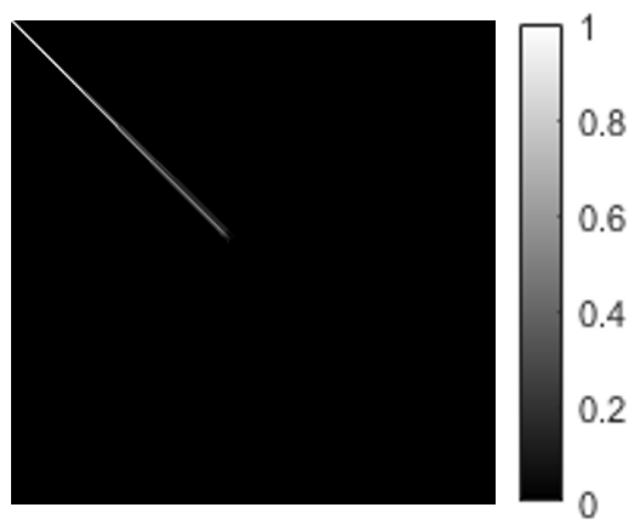

Fonte: Próprio autor. 


\subsection{Seleção de Parâmetros}

Inicialmente, as classificações foram feitas com os parâmetros propostos por Trardi e seus colaboradores (2018), porque esse trabalho apresentou a melhor acurácia da literatura.

Em segundo lugar, para reduzir o número de parâmetros usados, aqueles parâmetros obtidos pelos sinais diferenciados ou de dinâmica de ordem maior foram descartados. Também se verificou que o parâmetro $S D S D_{i}$ apresentava valores semelhantes ao $S D_{i}$ e $S D 2_{i}$ e, por isso, os parâmetros $S D_{i}$ e $S D 2_{i}$ foram descartados. Além disso, o parâmetro razão entre o máximo e o mínimo intervalo $R R$ foi acrescentado aos 13 parâmetros remanescentes dentre aqueles propostos por Trardi e seus colaboradores (2018). Dessa forma, obteve-se um novo conjunto de 14 parâmetros para classificação.

Por fim, um terceiro conjunto de parâmetros foi proposto. Todos os parâmetros descritos na seção 3.2 foram aplicados individualmente ao classificador linear, resultando em classificações individuais. Desses, os parâmetros semelhantes foram descartados. Foi feito um ranking das taxas de classificações e as 14 melhores foram mantidas. Em seguida, os parâmetros foram agrupados de $10 \mathrm{em} \mathrm{10.} \mathrm{A} \mathrm{melhor}$ combinação de 10 parâmetros foi escolhida por fornecer a maior acurácia. Esse conjunto de 10 parâmetros escolhidos forneceu melhores acurácias do que os dois conjuntos iniciais, na classificação por máquina de vetor de suporte. Por isso, ele foi usado também na classificação por rede neural artificial visando avaliar o impacto do tipo de classificador.

\subsection{Discriminante linear de Fisher (DL)}

O discriminante linear de Fisher (DL) é um classificador linear que busca projetar os dados em uma reta. Essa projeção é feita de forma que ocorra a maior separação possível entre as classes projetadas. [Duda; Hart, 1973]

A figura 17 exemplifica uma reta onde as projeções das classes representadas por e - estão bem separadas. As projeções do $\boldsymbol{\Lambda}$ ficam do lado esquerdo superior da reta

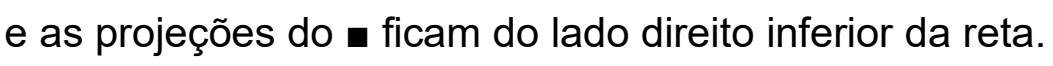


Figura 17: Exemplo de uma reta de projeção que separa as duas classes representadas por $\boldsymbol{\Delta}$ e $\mathbf{m}$. A reta que separa as classes está representada pela linha contínua e a reta de projeção pela linha tracejada.

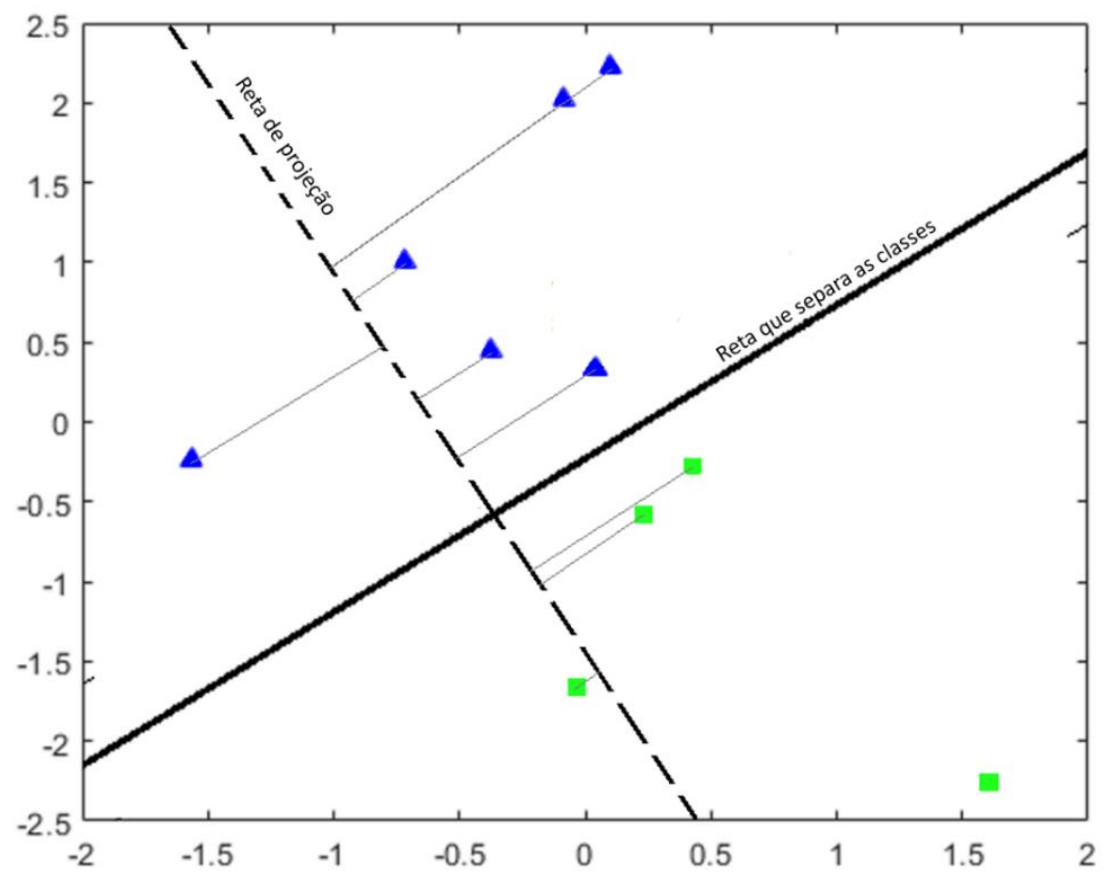

Fonte: Próprio autor.

Considera-se uma amostra de treinamento $\left\{\mathbf{x}_{\mathbf{i}}\right\}_{i=1}^{N}$, em que $\mathbf{x}_{\mathbf{i}}$ é um vetor de parâmetros de entrada e $N$ é o número de amostras. As classes de fibrilação atrial e normal são representadas por $X_{1}$ de quantidade $n_{1}$ e $X_{2}$ de quantidade $n_{2}$ respectivamente.

A projeção das amostras na reta é feita por

$$
y=\mathbf{w}^{T} \mathbf{x},
$$

em que $\mathbf{x}$ é o vetor de entrada, w é o vetor de projeção e y é a projeção do $\mathbf{x}$ na linha da direção de $\mathbf{w}$. As projeções $y_{i}$ dos vetores $\mathbf{x}_{i}$ pertencem ao conjunto $Y_{\mathbf{j}}$.

A magnitude de w não é importante porque ela apenas escalona o valor de $y$, mas a direção de w é importante. As projeções serão diferentes dependendo da direção de w e a direção desejada é aquela que gera a projeção das classes com a maior separação. [Duda; Hart, 1973] 
Uma forma de medir a separação entre as classes projetadas é a diferença entre as médias. A média $\mathbf{m}_{\mathbf{i}}$ das amostras da classe $i$ é dada por

$$
\mathbf{m}_{i}=\frac{1}{n_{i}} \sum_{\mathbf{x} \in X_{i}} \mathbf{x}
$$

a média dos pontos projetados é dada por

$$
\widetilde{\mathrm{m}}_{\mathrm{i}}=\frac{1}{n_{i}} \sum_{y \in X_{i}} y=\frac{1}{n_{i}} \sum_{\mathbf{x} \in X_{i}} \mathbf{w}^{T} \mathbf{x}=\mathbf{w}^{T} \mathbf{m}_{i}
$$

Desta forma, $\left|\widetilde{\mathrm{m}}_{1}-\widetilde{\mathrm{m}}_{2}\right|=\left|\mathbf{w}^{T}\left(\mathbf{m}_{1}-\mathbf{m}_{2}\right)\right|$. Para obter uma boa separação dos dados projetados a diferença das médias deve ser maior que o desvio-padrão de cada classe.

O espalhamento para os pontos projetados da classe $Y_{\mathrm{i}}$ é definido por

$$
\widetilde{\mathrm{s}}_{i}^{2}=\sum_{y \in X_{i}}\left(y-\widetilde{\mathrm{m}}_{\mathrm{i}}\right)^{2}
$$

e $\widetilde{s}_{1}^{2}+\widetilde{s}_{2}^{2}$ é o espalhamento dentro das classes (within-class) das amostras projetadas. O discriminante linear de Fisher é aquele que apresenta o máximo valor para a função

$$
J(\mathbf{w})=\frac{\left|\widetilde{\mathrm{m}}_{1}-\widetilde{\mathrm{m}}_{2}\right|^{2}}{\widetilde{\mathrm{s}}_{1}^{2}+\widetilde{\mathrm{s}}_{2}^{2}} .
$$

Para obter $J$ como uma função explícita de $\mathbf{w}$, são definidas as matrizes de espalhamento $S_{\mathrm{i}}$ e $S_{\mathrm{W}}$ por

$$
S_{i}=\sum_{\mathbf{x} \in X_{i}}\left(\mathbf{x}-\mathbf{m}_{1}\right)\left(\mathbf{x}-\mathbf{m}_{2}\right)^{T}
$$

e

$$
S_{W}=S_{1}+S_{2},
$$


de forma que

$$
\begin{gathered}
\widetilde{\mathrm{s}}_{i}^{2}=\mathbf{w}^{T} S_{i} \mathbf{w}, \\
\widetilde{\mathrm{s}}_{1}^{2}+\widetilde{\mathrm{s}}_{2}^{2}=\mathbf{w}^{T} S_{W} \mathbf{w}, \\
\left(\widetilde{\mathrm{m}}_{1}-\widetilde{\mathrm{m}}_{2}\right)^{2}=\mathbf{w}^{T} S_{B} \mathbf{w},
\end{gathered}
$$

em que a matriz de espalhamento entre classes é dada por

$$
S_{B}=\left(\mathbf{m}_{1}-\mathbf{m}_{2}\right)\left(\mathbf{m}_{1}-\mathbf{m}_{2}\right)^{T}
$$

A função a ser maximizada pode ser reescrita como

$$
J(\mathbf{w})=\frac{\mathbf{w}^{T} S_{B} \mathbf{w}}{\mathbf{w}^{T} S_{W} \mathbf{w}}
$$

O vetor de pesos $\mathbf{w}_{o}$ que otimiza a função é

$$
\mathbf{w}_{o}=S_{W}^{-1}\left(\mathbf{m}_{1}-\mathbf{m}_{2}\right) \text {. }
$$

E o limiar é dado por $y_{\text {limiar }}=\mathbf{w}_{o}{ }^{T} \mathbf{m}$, em que $\mathbf{m}$ é a média de todas as amostras.

Caso a projeção $y_{i}=\mathbf{w}_{o}^{T} \mathbf{x}_{i}$ for maior que o limiar $y_{\text {limiar }}$, então a amostra $i$ pertence à classe $X_{1} \mathrm{e}$, caso contrário, à $X_{2}$.

\subsection{Máquina de vetor de suporte (SVM)}

A máquina de vetor de suporte (SVM, Support Vector Machine) é um classificador que realiza o mapeamento não-linear dos parâmetros de entrada para um espaço de características de alta dimensionalidade. Após o mapeamento é feita a construção de um hiperplano que apresente a máxima margem entre as duas classes. A sua implementação é feita de acordo com as características dos dados de entrada. $O$ modelo mais simples é implementado quando os dados são linearmente separáveis e modelos mais complexos são implementados quando os dados não são linearmente separáveis. [Cristianini; Taylor, 2000; Haykin, 2001] 


\subsubsection{Hiperplano de caso linearmente separáveis}

Considera-se uma amostra de treinamento $\left\{\left(\mathbf{x}_{i}, d_{i}\right)\right\}_{i=1}^{N}$, em que $\mathbf{x}_{i}$ é o vetor de parâmetros de entrada, $d_{i}$ é a classe para i-ésima amostra e $\mathrm{N}$ é o número de amostras de treinamento. As classes de fibrilação atrial e normal são representadas por $d_{i}=+1$ e $d_{i}=-1$ respectivamente.

A equação do hiperplano que realiza a separação das classes é dada por

$$
\mathbf{w}^{T} \mathbf{x}+b=0,
$$

em que $\mathbf{x}$ é o vetor de entrada, $\mathbf{w}$ é o vetor de pesos e bé o viés.

A figura 18 demonstra um hiperplano de separação de duas classes. A separação entre o hiperplano e o vetor de treinamento mais próximo é denominada margem de separação $\rho$. $O$ hiperplano que apresenta o máximo $\rho$ é referido como hiperplano ótimo. [Haykin, 2001]

Figura 18: Exemplo de um hiperplano de separação, representada com linha contínua, de duas classes representadas por $\boldsymbol{\Delta}$ e $\mathbf{m}$, com os vetores de suporte em círculos e a margem de separação $\rho$.

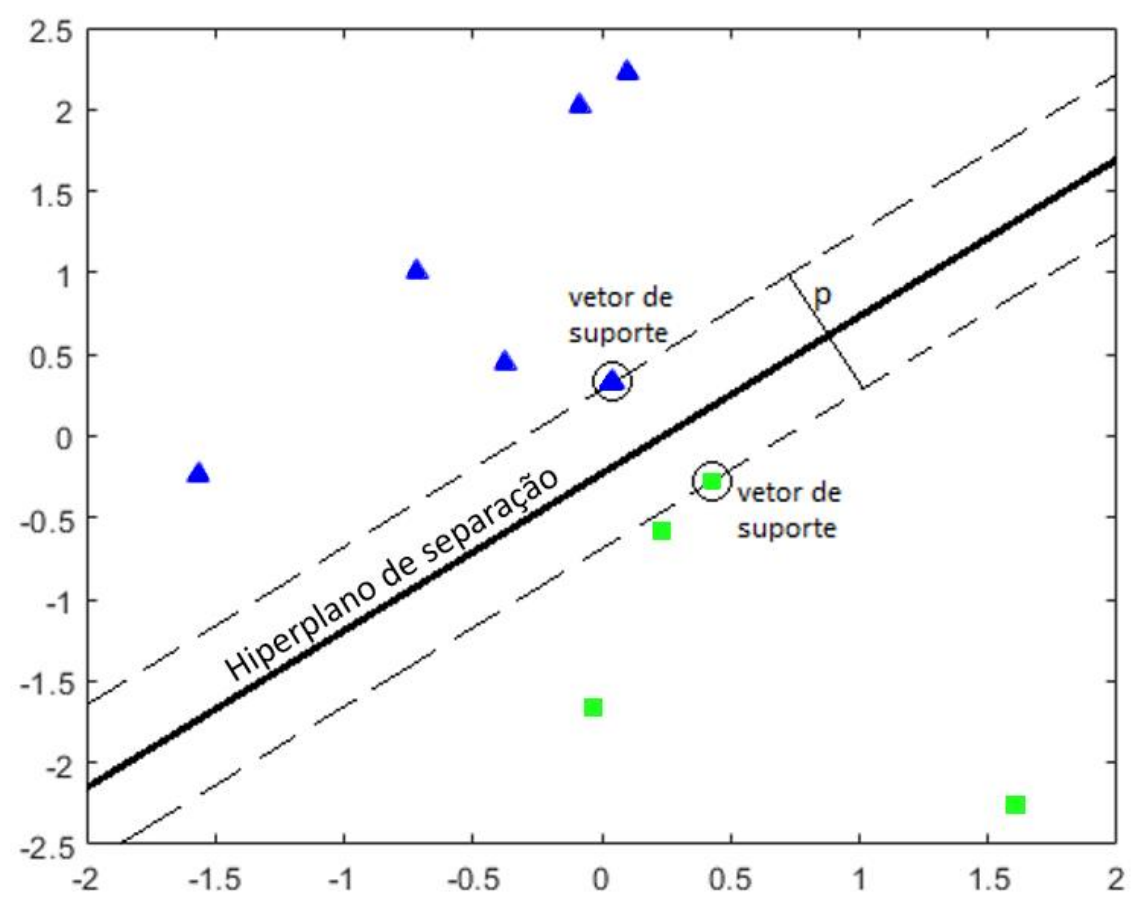

Fonte: Próprio autor. 
Sendo $\mathbf{w}_{\circ}$ e $b_{0}$ o vetor de peso e o viés ótimos, o hiperplano ótimo é definido por

$$
\mathbf{w}_{o}^{T} \mathbf{x}+b_{o}=0,
$$

e o par ( $\mathbf{w}_{\circ}$ e $\left.b_{0}\right)$ deve satisfazer a restrição:

$$
\begin{aligned}
\mathbf{w}_{o}^{T} \mathbf{x}_{i}+b_{o} & \geq 1 \text { para } d_{i}=+1 \\
\mathbf{w}_{o}^{T} \mathbf{x}_{i}+b_{o} & \leq 0, \text { para } d_{i}=-1
\end{aligned}
$$

Os dados de entrada $\mathbf{x}^{(s)}$ que satisfazem $\mathbf{w}_{o}^{T} \mathbf{x}_{i}+b_{o}= \pm 1$, são chamados de vetores de suporte. Por ser um caso de classes linearmente separáveis, não haverá casos intermediários em que $-1<\mathbf{w}_{o}^{T} \mathbf{x}_{i}+b_{o}<1$. [Haykin, 2001]

Para encontrar o hiperplano ótimo, é necessário minimizar a função

$$
\Phi(\mathbf{w})=\frac{1}{2} \mathbf{w}^{T} \mathbf{w}
$$

sujeitas à restrição

$$
d_{i}\left(\mathbf{w}^{T} \mathbf{x}_{i}+b\right) \geq 1 \text { para } i=1,2, \ldots, N
$$

Este problema pode ser resolvido com o método dos multiplicadores de Lagrange.

Os multiplicadores de Lagrange ótimos $\left(\alpha_{\circ}\right)$ maximizam a função

$$
Q(\alpha)=\sum_{i=1}^{N} \alpha_{\mathrm{i}}-\frac{1}{2} \sum_{i=1}^{N} \sum_{i=1}^{N} \alpha_{\mathrm{i}} \alpha_{\mathrm{j}} d_{i} d_{j} \mathbf{x}_{i}^{T} \mathbf{x}_{j}
$$

sujeita às restrições

$$
\sum_{i=1}^{N} \alpha_{i} d_{i}=0
$$

e

$$
\alpha_{\mathrm{i}} \geq 0 \text { para } i=1,2, \ldots, N .
$$


O vetor ótimo de pesos $\mathbf{w}_{0}$ é obtido por

$$
\mathbf{w}_{o}=\sum_{i=1}^{N} \alpha_{\mathrm{o}, \mathrm{i}} d_{i} \mathbf{x}_{i}
$$

e o viés por

$$
b_{o}=1-\mathbf{w}_{o}^{T} \mathbf{x}^{(\mathrm{s})} \text { para } d^{(s)}=1,
$$

em que $\mathbf{x}^{(s)}$ são os vetores de suporte que apresentam $d^{(s)}=1$.

\subsubsection{Hiperplano de caso linearmente não-separáveis}

Quando os dados não são linearmente separáveis, não é possível construir um hiperplano de separação sem que ocorra erros de classificação. Para resolver este problema é utilizada uma margem de separação suave, com a introdução de uma variável $\left\{\xi_{i}\right\}_{i=1}^{N}$. [Haykin, 2001]

O hiperplano fica sujeito à restrição

$$
d_{i}\left(\mathbf{w}^{T} \mathbf{x}_{i}+b\right) \geq 1-\xi_{i} \text { para } i=1,2, \ldots, N
$$

E para encontrar o hiperplano ótimo é necessário minimizar a função

$$
\Phi(\mathbf{w})=\frac{1}{2} \mathbf{w}^{T} \mathbf{w}+C \sum_{i=1}^{N} \xi_{i}
$$

com as restrições

$$
d_{i}\left(\mathbf{w}^{T} \mathbf{x}_{i}+b\right) \geq 1-\xi_{i} \text { para } i=1,2, \ldots, N
$$

e

$$
\xi_{i} \geq 0 \text { para todo } i
$$

em que $C$ é um parâmetro selecionado pelo usuário que está relacionado à complexidade da máquina e ao número de amostras não separáveis. Um dos métodos 
para determinar o parâmetro $C$ ótimo é experimental. Outro método de estimar o valor do parâmetro $C$ é por meio da dimensão Vapnik-Chervonekis $h$ dada por

$$
h \leq \min \left\{\left[\frac{D^{2}}{\rho^{2}}\right], m_{0}\right\}+1,
$$

em que $D$ representa o diâmetro da menor esfera que contém todos os vetores de entrada $\left.\left\{\mathbf{x}_{i}\right\}_{i=1}^{N}, \Gamma \cdot\right\rceil$ representa teto do valor, $\rho=\frac{2}{\left\|\mathbf{w}_{o}\right\|}$ é a margem de separação e $m_{0}$ é a dimensionalidade do espaço de entrada. [Haykin, 2001]

Neste mestrado, adotou-se C unitário como um valor padrão.

Utilizando o método dos multiplicadores de Lagrange, os multiplicadores ótimos $\left(\alpha_{0}\right)$ maximizam a função

$$
Q(\alpha)=\sum_{i=1}^{N} \alpha_{\mathrm{i}}-\frac{1}{2} \sum_{i=1}^{N} \sum_{i=1}^{N} \alpha_{\mathrm{i}} \alpha_{\mathrm{j}} d_{i} d_{j} \mathbf{x}_{i}^{T} \mathbf{x}_{j}
$$

sujeita às restrições

$$
\sum_{i=1}^{N} \alpha_{\mathrm{i}} d_{i}=0
$$

e

$$
0 \geq \alpha_{\mathrm{i}} \geq C \text { para } i=1,2, \ldots, N
$$

O vetor de peso ótimo é dado por

$$
\mathbf{w}_{o}=\sum_{i=1}^{N_{s}} \alpha_{\mathrm{o}, \mathrm{i}} d_{i} \mathbf{x}_{i}
$$

em que $N_{s}$ é o número de vetores de suporte.

O viés ótimo é obtido por meio de uma amostra qualquer $\left(\mathbf{x}_{i}, d_{i}\right)$ do conjunto de treinamento para a qual $0<\alpha_{o, i}<C$. Com isso, tem-se $\xi_{i}=0$, que resulta em

$$
d_{i}\left(\mathbf{w}_{o}^{T} \mathbf{x}_{i}+b_{o}\right)=1 .
$$




\subsubsection{Transformação para espaço de maior dimensão}

Além de usar o hiperplano de separação, a SVM mapeia os parâmetros de entrada em um espaço de maior dimensionalidade. Ao realizar esse mapeamento é possível que alguns casos de classes linearmente não separáveis se tornem linearmente separáveis nesse novo espaço. [Cristianini; Taylor, 2000; Haykin, 2001]

O conjunto de funções de transformações $\left\{\varphi_{j}\right\}_{j=1}^{m_{1}}$, em que $m_{1}$ é a dimensão do novo espaço, é definido para todo $j$. Sendo $\boldsymbol{\varphi}(\mathbf{x})=\left[\varphi_{1}(\mathbf{x}), \varphi_{2}(\mathbf{x}), \ldots, \varphi_{m_{1}}(\mathbf{x})\right]^{T}$.

Com esses conjuntos de transformações pode-se definir um hiperplano da forma

$$
\mathbf{w}^{T} \boldsymbol{\varphi}(\mathbf{x})+b=0 .
$$

Com esta alteração, os multiplicadores de Lagrange ótimos maximizam a função

$$
Q(\alpha)=\sum_{i=1}^{N} \alpha_{\mathrm{i}}-\frac{1}{2} \sum_{i=1}^{N} \sum_{i=1}^{N} \alpha_{\mathrm{i}} \alpha_{\mathrm{j}} d_{i} d_{j} \boldsymbol{\varphi}\left(\mathbf{x}_{i}\right)^{T} \boldsymbol{\varphi}\left(\mathbf{x}_{j}\right) .
$$

O termo $\boldsymbol{\varphi}\left(\mathbf{x}_{i}\right)^{T} \boldsymbol{\varphi}\left(\mathbf{x}_{j}\right)$ pode ser representado por $K\left(\mathbf{x}_{i}, \mathbf{x}_{j}\right)$, que é denominado núcleo de produto interno (kerne). A função a ser maximizada se torna

$$
Q(\alpha)=\sum_{i=1}^{N} \alpha_{\mathrm{i}}-\frac{1}{2} \sum_{i=1}^{N} \sum_{i=1}^{N} \alpha_{\mathrm{i}} \alpha_{\mathrm{j}} d_{i} d_{j} K\left(\mathbf{x}_{i}, \mathbf{x}_{j}\right),
$$

sujeita as restrições

$$
\sum_{i=1}^{N} \alpha_{i} d_{i}=0
$$

e

$$
\alpha_{\mathrm{i}} \geq 0 \text { para } i=1,2, \ldots, N .
$$


Os valores ótimos para o vetor de pesos são dados por

$$
\mathbf{w}_{o}=\sum_{i=1}^{N_{s}} \alpha_{\mathrm{o}, \mathrm{i}} d_{i} \boldsymbol{\varphi}\left(\mathbf{x}_{i}\right)
$$

e o viés ótimo por

$$
b_{o}=\sum_{i=1}^{N} \alpha_{\mathrm{o}, \mathrm{i}} d_{i}
$$

Um dos núcleos de produto interno mais utilizado é a função de base radial com sua expressão dada por

$$
K\left(\mathbf{x}_{i}, \mathbf{x}_{j}\right)=\exp \left(-\frac{1}{2 \sigma^{2}}\left\|x_{i}-x_{j}\right\|^{2}\right)
$$

em que $\sigma$ é a largura do núcleo definido pelo usuário.

O núcleo de produto interno para máquina de aprendizagem polinomial é dado por

$$
K\left(\mathbf{x}_{i}, \mathbf{x}_{j}\right)=\left(\mathbf{x}_{i}^{T} \mathbf{x}_{j}+1\right)^{p}
$$

em que a potência p é definida pelo usuário.

\subsection{Rede neural artificial (RNA)}

O funcionamento das redes neurais artificiais é inspirado no funcionamento dos circuitos cerebrais. Busca-se obter um sistema que possa realizar as mesmas tarefas realizadas pelo cérebro, como a classificação de padrões, a tomada de decisões, as previsões e os controles adaptativos. Esse sistema seria formado por neurônios, também denominado de nós, em uma ou mais camadas com ligações entre eles por meio de pesos e vieses (ou limiares). [Fine, 1999]

A modelagem do neurônio da rede neural é baseada em características existentes no neurônio de um mamífero. A transmissão de dados de um neurônio é unidirecional, apresentando múltiplas entradas por meio de dendritos e uma única saída conduzida 
via axônio, que pode ser conectada a vários outros neurônios. A resposta do neurônio é do tipo "tudo ou nada" e é determinada pelo somatório das entradas. [Fine, 1999]

\subsubsection{Modelo matemático dos neurônios (Perceptron)}

A saída $y$ de um neurônio é relacionada às múltiplas entradas $\left[x_{1}, \ldots, x_{n}\right]$, à força das conexões sinápticas $w=\left[w_{1}, \ldots, w_{n}\right]$ e ao limiar de disparo $b$, por meio de uma função não linear $f$. [Haykin, 2009]

$$
y=f\left(\sum_{1}^{n} w_{i} x_{i}-b\right)=f(\underline{w} \cdot \underline{x}-b) .
$$

Figura 19: Esboço de um neurônio com as suas entradas e saída. As entradas $\left[x_{1}, \ldots, x_{n}\right]$ multiplicadas pelos seus respectivos pesos $\left[w_{1}, \ldots, w_{n}\right]$ e 0 viés $b_{1}$ são introduzidos no neurônio com a função de ativação $F_{1}$ resultando na saída $y$.

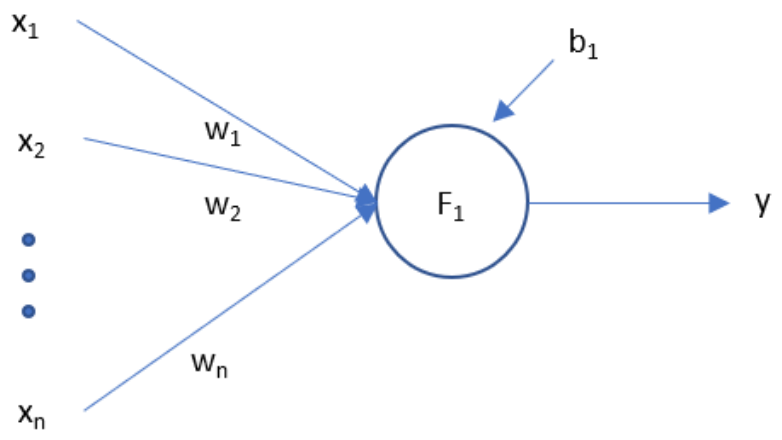

Fonte: Próprio autor.

Há duas funções que apresentam a resposta característica do tipo "tudo ou nada" determinada pelo limiar, que são a função degrau unitário, também denominada função de Heaviside (U)

$$
f(z)=U(z-\boldsymbol{b})=\left\{\begin{array}{l}
1, \quad \text { se } z \geq b \\
0, \quad \text { caso contrário }
\end{array}\right.
$$

e a correspondente função sinal (ou sign)

$$
\operatorname{sign}(z)=2 U(z)-1
$$

em que $z=\sum_{1}^{n} w_{i} x_{i}$. 
Quando se considera que o neurônio apresenta uma resposta gradual em vez de uma resposta do tipo "tudo ou nada", são usadas duas funções com a forma de sigmoide: a função logística $(F)$

$$
F l(z)=\frac{1}{1+e^{-\alpha z}}
$$

e a função tangente hiperbólica (tanh)

$$
\tanh (\alpha z)=\frac{e^{\alpha z}-e^{-\alpha z}}{e^{\alpha z}+e^{-\alpha z}}=2 F l(2 z)-1 .
$$

Para pequenos valores de $z$, a função logística e a tangente hiperbólica são praticamente lineares. Por isso, redes neurais com pequenas excitações dos nós formados com essas funções apresentarão características semelhantes aos sistemas lineares. [Haykin, 2009]

\subsubsection{Redes neurais de alimentação direta (feedforward)}

A arquitetura de uma rede neural de alimentação direta é definida por um grafo orientado acíclico e a função escolhida do nó. A camada inicial $L_{0}$ contém os nós de entrada e a i-ésima camada $L_{i}$ apresenta os nós com entradas ligadas aos nós de $L_{j}$ em que $j<i$. A camada final apresenta os nós de saída. Um caso específico é o perceptron de múltiplas camadas (MLP, do inglês multilayer perceptron) em que cada camada tem ligações somente com a sua camada anterior. [Fine, 1999]

A figura 20 mostra um esboço de uma rede neural do tipo perceptron de múltiplas camadas e a figura 21 apresenta um exemplo específico da arquitetura simplificada de uma rede implementada com duas camadas internas de 21 neurônios em cada camada e uma camada de saída com apenas um neurônio. 
Figura 20: Esboço de uma rede neural do tipo perceptron de múltiplas camadas. Os triângulos representam as entradas $\left[x_{1}, \ldots, x_{n}\right]$ no lado esquerdo e as saídas $\left[y_{1}, \ldots, y_{m}\right]$ no lado direito. Os círculos representam os neurônios com as suas funções $\left[F_{1}^{1}, \ldots, F_{L}^{S_{L}}\right]$, em que $\left[S_{1}, \ldots, S_{L}\right]$ são a quantidade de neurônios nas camadas $1,2, \ldots, \mathrm{L}$ respectivamente.

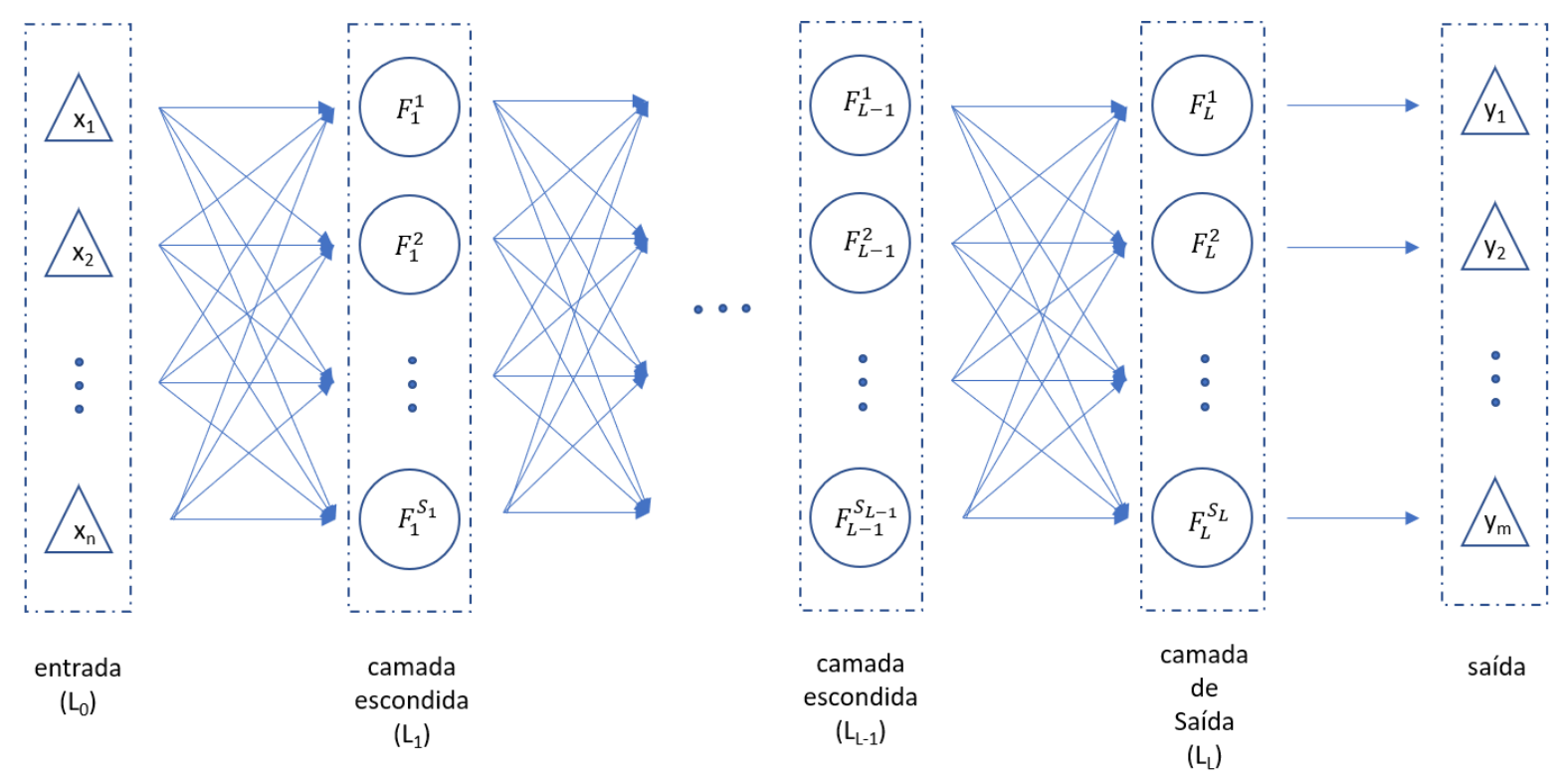

Fonte: Próprio autor.

Figura 21: Arquitetura da rede neural implementada com 21 neurônios nas camadas internas.

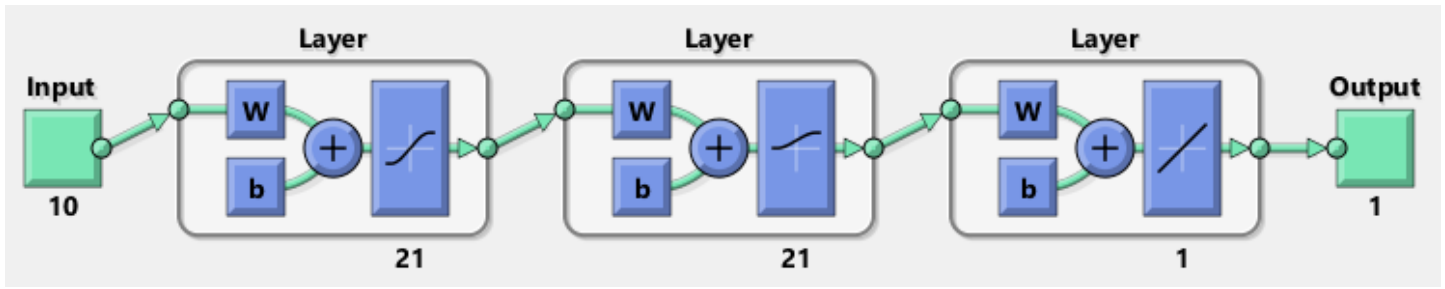

Fonte: Próprio autor.

\subsubsection{Método de Backpropagation}

Dada uma entrada $x=\left[x_{1}, \ldots, x_{n}\right]$ na rede neural do tipo feedforward, a i-ésima saída $a_{o}(i)$ da camada de entrada $\left(L_{0}\right)$ será [Fine, 1999]

$$
a_{o}(i)=x_{i} .
$$


Considerando-se uma rede neural MLP com $S_{j}$ neurônios na camada j, em que $a_{j}(i) e b_{j}(i)$ são respectivamente a saída e o viés do nó $i$ da camada $j$ e $w_{j}(i, k)$ é o peso na ligação entre o nó $i$ na camada $j$ com o nó $k$ na camada $j-1$, a entrada do nó $i$ na camada $j$ é dada por [Fine, 1999]

$$
c_{j}(i)=\sum_{k=1}^{s_{j}} w_{j}(i, k) a_{j-1}(k)+b_{j}(i) .
$$

E a saída do neurônio $i$ é [Fine, 1999]

$$
a_{j}(i)=F_{j}^{i}\left(c_{j}(i)\right)
$$

em que $F_{j}^{i}(z)$ é a função do i-ésimo nó da j-ésima camada.

Se houver apenas um nó na camada de saída $\left(L_{L}\right)$, então haverá apenas uma saída $y$ dada por [Fine, 1999]

$$
a_{L}(1)=y \text {. }
$$

Caso a saída desejada seja $t$, o erro da rede será dado por [Fine, 1999]

$$
\frac{1}{2}\|y-t\|^{2}=\frac{1}{2}(y-t)^{2}=e^{2}=\varepsilon
$$

Para um conjunto de treinamento T, o erro $\varepsilon_{T}$ seria dado por [Fine, 1999]

$$
\varepsilon_{T}=\frac{1}{2} \sum_{i=1}^{N}\left\|\underline{y_{i}}-\underline{t_{i}}\right\|^{2},
$$

em que $\underline{y}_{i}$ e $\underline{t}_{i}$ são respectivamente os vetores de saída da rede e valores desejados para a i-ésima amostra de treinamento e $N$ é o número total de amostras.

O vetor de gradiente $\underline{g}(\underline{w})$ é definido pelas derivadas parciais do erro [Fine, 1999]

$$
\underline{g}(\underline{w})=\left.\nabla \varepsilon_{T}\right|_{\underline{w}}=\left.\frac{\partial \varepsilon_{T}}{\partial w_{i}}\right|_{\underline{w}}
$$


e a matriz hessiana $\mathbf{H}(\underline{w})$ pelas derivadas parciais de segunda ordem

$$
\mathbf{H}(\underline{w})=\nabla^{2} \varepsilon_{T}(\underline{w}),
$$

em que o elemento $H_{i j}$ da i-ésima linha e j-ésima coluna da matriz hessiana é dado por

$$
H_{i j}=\frac{\partial^{2} \varepsilon_{T}}{\partial w_{i} \partial w_{j}}
$$

O método de retropropagação (backpropagation) fornece um método de estimar o vetor de gradiente que é utilizado para ajustar os parâmetros, a partir de duas etapas. Durante a primeira etapa a entrada é fornecida e propagada pela rede para o cálculo dos valores de $a_{j}(i)$ em cada neurônio. Na segunda etapa, são calculados os erros de forma recursiva. Com os valores calculados nas duas etapas é possível estimar o vetor de gradiente usado para ajustar os parâmetros. [Fine, 1999]

O gradiente do erro $\varepsilon$ em função dos pesos $w_{j}(i, k)$ ou vieses $b_{j}(i)$ é dado por [HechtNielsen, 1989]

$$
\frac{\partial \varepsilon}{\partial w_{j}(i, k)}=\frac{\partial \varepsilon}{\partial c_{j}(i)} \frac{\partial c_{j}(i)}{\partial w_{j}(i, k)}=\frac{\partial \varepsilon}{\partial c_{j}(i)} a_{j-1}(k),
$$

para

$$
\frac{\partial \varepsilon}{\partial b_{j}(i)}=\frac{\partial \varepsilon}{\partial c_{j}(i)} .
$$

Denominando a contribuição da excitação $c_{j}(i)$ do nó $i$ na camada $j$ para o erro como [Hecht-Nielsen, 1989]

$$
\delta_{j}(i)=\frac{\partial \varepsilon}{\partial c_{j}(i)^{\prime}}
$$

as expressões anteriores podem ser rescritas como

$$
\frac{\partial \varepsilon}{\partial c_{j}(i)} a_{j-1}(k)=\delta_{j}(i) a_{j-1}(k) .
$$


Se a camada jé escondida, então o erro depende de $a_{j}(i)$ somente pela sua influência na camada $j+1$ em que ela é a entrada, resultando em [Hecht-Nielsen, 1989]

$$
\frac{\partial \varepsilon}{\partial a_{j}(i)}=\sum_{k=1}^{s_{j+1}} \frac{\partial \varepsilon}{\partial c_{j+1}(k)} \frac{\partial c_{j+1}(k)}{\partial a_{j}(i)}=\sum_{k=1}^{s_{j+1}} \delta_{j+1}(k) w_{j+1}(k, i) .
$$

Como o erro depende de $c_{j}(i)$ somente por $a_{j}(i)$,

$$
\delta_{j}(i)=\frac{\partial \varepsilon}{\partial a_{j}(i)} \frac{\partial a_{j}(i)}{\partial c_{j}(i)}=F_{j}^{i}\left(c_{j}(i)\right) \frac{\partial \varepsilon}{\partial a_{j}(i)}
$$

e combinando com o resultado anterior tem-se que

$$
\delta_{j}(i)=F_{j}^{i}\left(c_{j}(i)\right) \sum_{k=1}^{s_{j+1}} \delta_{j+1}(k) w_{j+1}(k, i)
$$

para $i<L$. [Hecht-Nielsen, 1989]

A condição final, para qual foi iniciada a retropropagação é provida por [Fine, 1999]

$$
\delta_{L}(1)=F_{L}^{1}\left(c_{L}(1)\right)\left(a_{L}(1)-t\right)
$$

A estimativa do gradiente é feita por conjuntos de etapa direta (forward) seguidas de etapas de retorno (backward). Na etapa direta são determinadas as saídas $a_{j}(i)$ e as entradas $c_{j}(i)$ dos neurônios obtidas para o conjunto de treinamento. Na etapa de retorno são determinados os $\delta_{j}(i)$. Com a combinação dos resultados é determinado o gradiente que é utilizado para ajustar os pesos. [Fine, 1999]

O treinamento da rede consiste em determinar o vetor de novos parâmetros $\underline{w}_{k+1}$ por meio da direção $\underline{d}_{k}$ e a taxa de aprendizagem $\alpha_{\mathrm{k}}$, [Hecht-Nielsen, 1989]

$$
\underline{w}_{k+1}=\underline{w}_{k}+\alpha_{\mathrm{k}} \underline{d}_{k}
$$

Fazendo a aproximação de primeira ordem $f(x)-f\left(x_{0}\right) \approx f^{\prime}\left(x_{0}\right)\left(x-x_{0}\right)$ para os valores sucessivos de erros com ajuste dos parâmetros seria obtido [Fine, 1999]

$$
\varepsilon(k+1)-\varepsilon(k) \approx \underline{g}\left(\underline{w}_{k}\right)^{T}\left(\underline{w}_{k+1}-\underline{w}_{k}\right)=\alpha_{\mathrm{k}} \underline{g}\left(\underline{w}_{k}\right)^{T} \underline{d}_{k} .
$$


Como se deseja que o erro diminua com a alteração dos parâmetros, deve se ter [Fine, 1999]

$$
\alpha_{\mathrm{k}} \underline{g}\left(\underline{w}_{k}\right)^{T} \underline{d}_{k}<0
$$

Uma forma de satisfazer a condição é ter ak positivo e [Fine, 1999]

$$
\underline{d}_{k}=-\mathbf{M}_{k} \underline{g}\left(\underline{w}_{k}\right)
$$

em que $\mathbf{M}_{k}$ é uma matriz positiva definida. No caso do método de Newton, $\mathbf{M}_{k}$ seria a inversa da matriz hessiana. Mas poderia ser uma outra matriz, como no método de Levenberg-Marquadt.

\subsubsection{Treinamento de redes neurais de alimentação direta pelo método de Marquadt}

O algoritmo de Levenberg-Marquadt (LMA) utiliza do fato de que a função de erro se dá pela soma quadrática [Marquadt, 1963; Fine, 1999]

$$
\varepsilon_{T}=\frac{1}{2} \sum_{i=1}^{n}\left(y_{i}-t_{i}\right)^{2}=\frac{1}{2} \sum_{i=1}^{n} e_{i}^{2}
$$

em que $n$ é a quantidade de amostras no conjunto de treinamento.

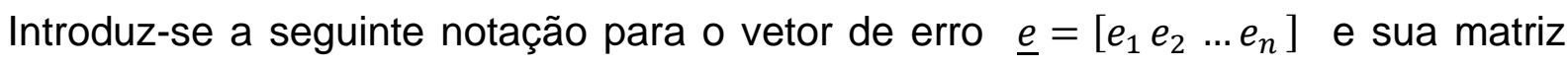
Jacobiana $\mathbf{J}$, cujos elementos são dados por

$$
J_{i j}=\frac{\partial \varepsilon}{\partial w_{i}}
$$

em que $i=1,2, \ldots, p ; p$ é a quantidade de parâmetros da rede; $j=1,2, \ldots, n ; n$ é a quantidade de amostras no conjunto de treinamento. A Jacobiana $\mathbf{J}$ é uma matriz $p \mathrm{x}$ $n$, em que todos os elementos são calculados diretamente pelo método de retropropagação. 
O vetor de gradiente $\underline{g}$ para o erro quadrático pode ser expressa como [Marquadt, 1963; Fine, 1999]

$$
\underline{g}(\underline{w})=\sum_{i=1}^{n} e_{i} \nabla e_{i}(\underline{w})=\mathbf{J} \underline{e} .
$$

A matriz hessiana $\mathbf{H}$ tem elementos [Marquadt, 1963; Fine, 1999]

$$
\begin{gathered}
H_{i j}=\frac{\partial^{2} \varepsilon_{T}}{\partial w_{i} \partial w_{j}}=\frac{1}{2} \sum_{k=1}^{n} \frac{\partial^{2} e_{k}^{2}}{\partial w_{i} \partial w_{j}}=\sum_{k=1}^{n}\left[e_{k} \frac{\partial^{2} e_{k}}{\partial w_{i} \partial w_{j}}+\frac{\partial e_{k}}{\partial w_{i}} \frac{\partial e_{k}}{\partial w_{j}}\right] \\
H_{i j}=\sum_{k=1}^{n}\left[e_{k} \frac{\partial^{2} e_{k}}{\partial w_{i} \partial w_{j}}+J_{i k} J_{j k}\right] .
\end{gathered}
$$

Define-se

$$
\mathbf{D}=\sum_{i=1}^{n} e_{i} \nabla^{2} e_{i}
$$

e obtém-se a expressão [Marquadt, 1963; Fine, 1999]

$$
\mathbf{H}(\underline{w})=\mathbf{J}^{T}+\mathbf{D} .
$$

O algoritmo de Levenberg-Marquadt é um algoritmo decrescente que aproxima a matriz $\mathbf{D}$ por uma matriz identidade I multiplicada por um valor escalar $\epsilon$, resultando em [Marquadt, 1963; Fine, 1999]

$$
\mathbf{M}_{k}=\left[\mathbf{J} \mathbf{J}^{T}+\epsilon \mathbf{I}\right]^{-\mathbf{1}} .
$$

Os pesos são atualizados pela relação

$$
\underline{w}_{k+1}=\underline{w}_{k}-\alpha_{k} \mathbf{M}_{k} \underline{g}\left(\underline{w}_{k}\right),
$$

em que $\alpha_{k}$ é a taxa de aprendizagem.

Para valores de $\epsilon$ próximos de zero, o algoritmo de Levenberg-Marquadt se aproxima do método de Newton usando a matriz hessiana aproximada. 


\subsection{Rede neural convolucional (CNN do inglês Convolutional Neural Network)}

As redes neurais convolucionais são usadas usualmente para reconhecimento de objetos, sendo formados essencialmente por camadas de convolução, camadas de pooling e camadas totalmente conectadas (Fully Conected).

\subsubsection{Camadas de Convolução}

A camada de convolução é a camada em que se realiza a transformação da matriz de pixel em outra matriz de características por meio de um núcleo (kernel). É necessário definir previamente o tamanho do kernel e a distância entre os centros (stride). Há ocorrência de sobreposição quando o valor da distância entre os centros é menor que o tamanho do kernel. [Goodfellow; Bengio; Courville, 2016]

Além do tamanho do kernel e da distância entre os centros, também se deve definir se uma borda será adicionada nas extremidades e, caso seja adicionada, qual será o valor utilizado. Utilizar o preenchimento (padding) permite que não haja redução ou que ocorra apenas uma pequena redução no tamanho da matriz analisada. [Goodfellow; Bengio; Courville, 2016]

A figura 22 ilustra o processo de convolução de uma imagem em escala de cinza de tamanho $4 \times 4$ com um kernel de tamanho $2 \times 2$ com distância entre os centros de valor 1 sem preenchimento.

Existem dois tipos de convolução, um com Kernel refletido e outro com Kernel não refletido. Nesse texto, utiliza-se a correlação cruzada que equivale à convolução com o Kernel não refletido [Goodfellow; Bengio; Courville, 2016]

$$
a^{i}(x, y)=I(x, y) * K^{i}(x, y)=\sum_{m=1}^{M} \sum_{n=1}^{N} I(x+m, y+n) K^{i}(m, n),
$$

em que $I(x, y)$ é a imagem com $\mathrm{M}$ linhas e $\mathrm{N}$ colunas e $K^{i}(x, y)$ é o i-ésimo kernel 
Figura 22: Exemplo de convolução com kernel de tamanho 2x2 com distância entre os centros de valor 1 e sem padding.
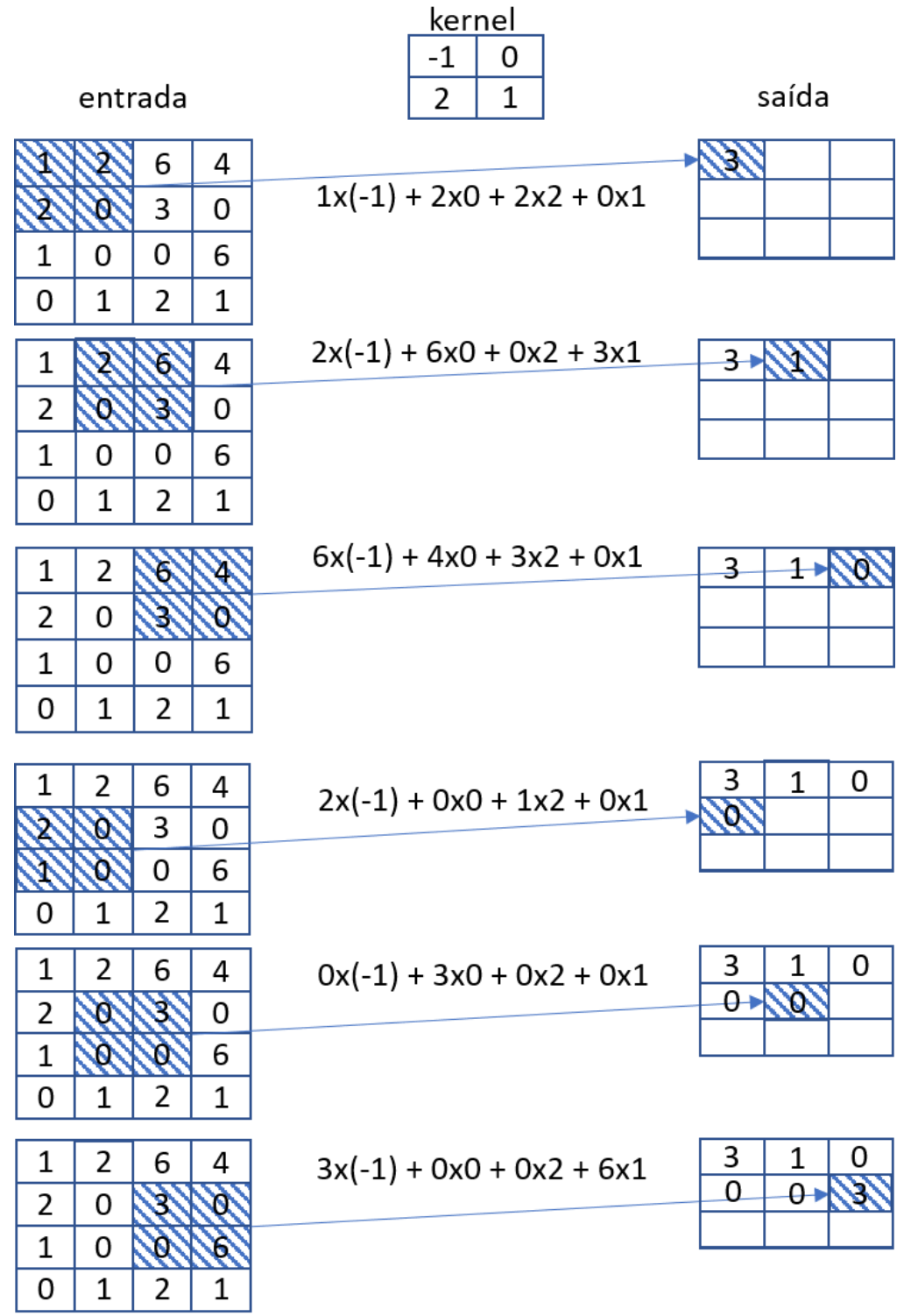

\begin{tabular}{|c|c|c|c|c|c|c|c|}
\hline 1 & 2 & 6 & 4 & \multirow{2}{*}{$1 \times(-1)+0 \times 0+0 \times 2+1 \times 1$} & 3 & 1 & 0 \\
\hline 2 & 0 & 3 & 0 & & 0 & 0 & 6 \\
\hline & & 0 & 6 & & & & \\
\hline & 4 & 2 & 1 & & & & \\
\hline
\end{tabular}

\begin{tabular}{|c|c|c|c|c|c|c|c|}
\hline 1 & 2 & 6 & 4 & \multirow{3}{*}{$0 \times(-1)+0 \times 0+1 \times 2+2 \times 1$} & \multirow{2}{*}{\multicolumn{2}{|c|}{$\frac{1}{0}$}} & \multirow{2}{*}{$\begin{array}{l}0 \\
6\end{array}$} \\
\hline 2 & 0 & 3 & 0 & & 0 & & \\
\hline 1 & (a) & a & 6 & & 0 & (4) & \\
\hline 0 & (1) & (2) & 1 & & & & \\
\hline
\end{tabular}

\begin{tabular}{|c|c|c|c|c|c|c|c|}
\hline 1 & 2 & 6 & 4 & \multirow{3}{*}{$0 \times(-1)+6 \times 0+2 \times 2+1 \times 1$} & 3 & 1 & 0 \\
\hline 2 & 0 & 3 & 0 & & 0 & 0 & 6 \\
\hline 1 & 0 & (2) & (6) & & 0 & & (5) \\
\hline 0 & 1 & 2 & (1) & & & & \\
\hline
\end{tabular}

Fonte: Próprio autor. 


\subsubsection{Camadas de Pooling}

A camada de Pooling reduz a dimensionalidade da entrada por um fator pré-definido. Ela serve para selecionar as características. A imagem de entrada é separada em sub-regiões, normalmente sem sobreposição. De cada sub-região se extrai um valor. Usualmente são extraídos o valor máximo ou o valor médio chamados de Max-Pooling e Avg-Pooling respectivamente. [Goodfellow; Bengio; Courville, 2016]

Figura 23: Exemplo de Avg-Pooling com o resultado da Figura 22 como entrada. O tamanho da unidade de pooling é de $2 \times 2$ com o espaçamento de 1 , ocorrendo sobreposição.

entrada saída
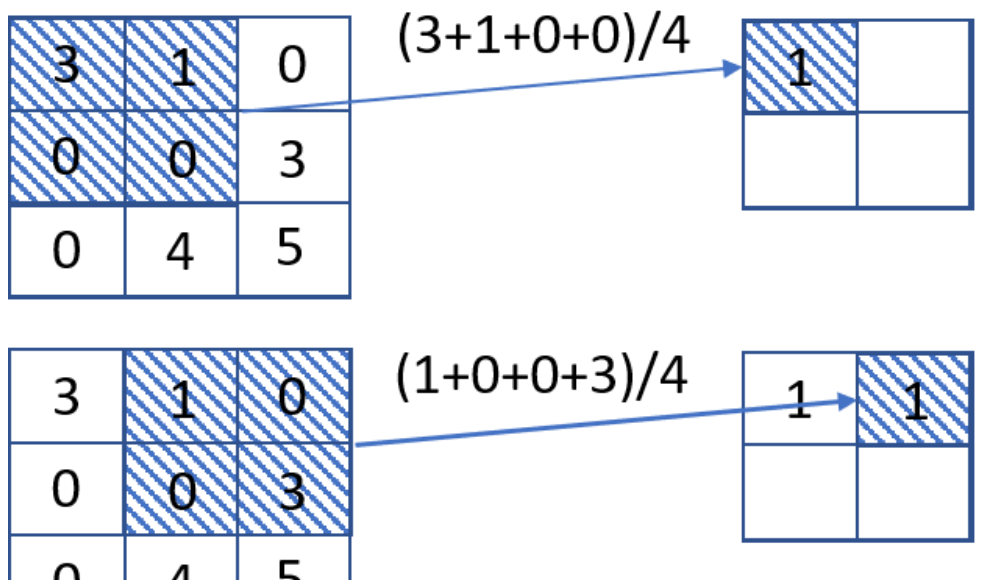

\begin{tabular}{l|l|l|}
0 & 4 & 5 \\
\hline
\end{tabular}
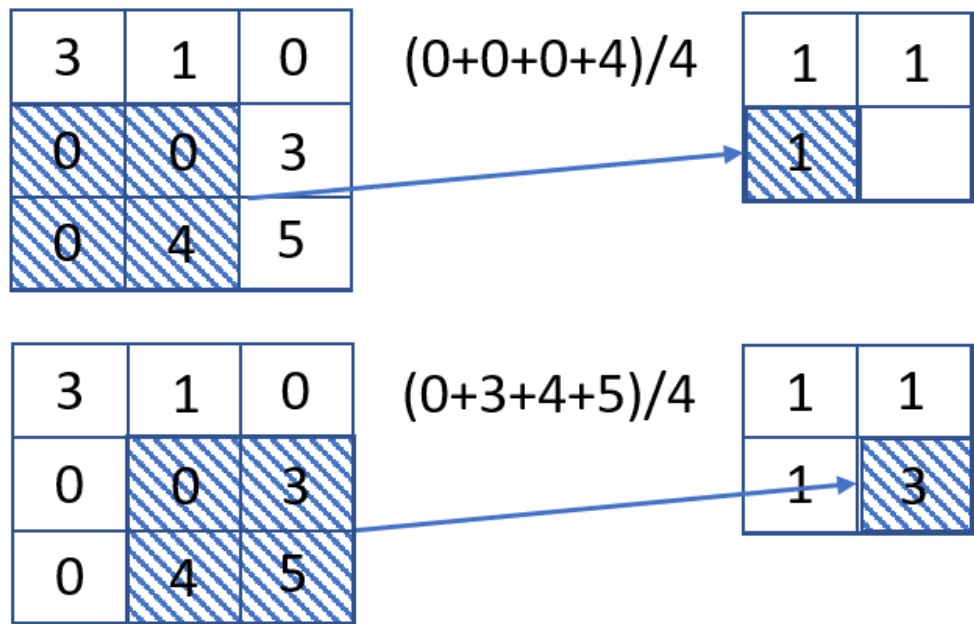

Fonte: Próprio autor. 
Figura 24: Exemplo de Max-Pooling com o resultado da Figura 22 como entrada. O tamanho da unidade de pooling é de $2 \times 2$ com o espaçamento de 1 , ocorrendo sobreposição.

entrada
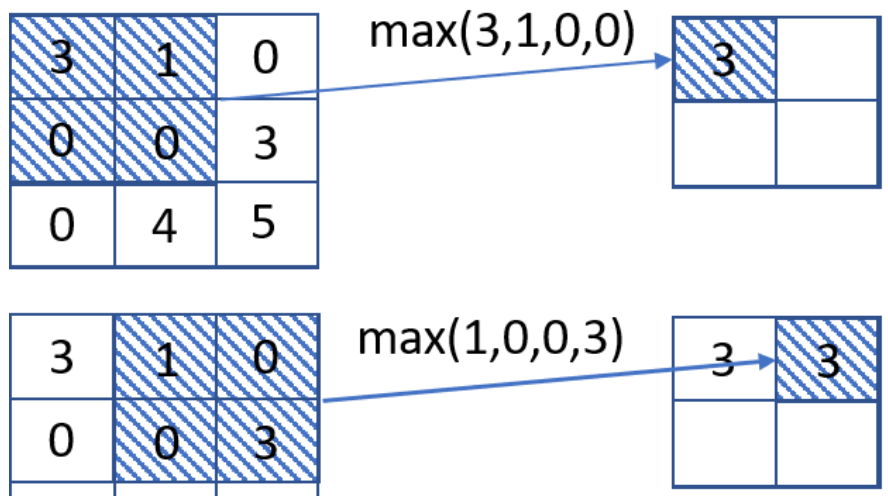

\begin{tabular}{l|l|l|}
0 & 4 & 5 \\
\hline
\end{tabular}
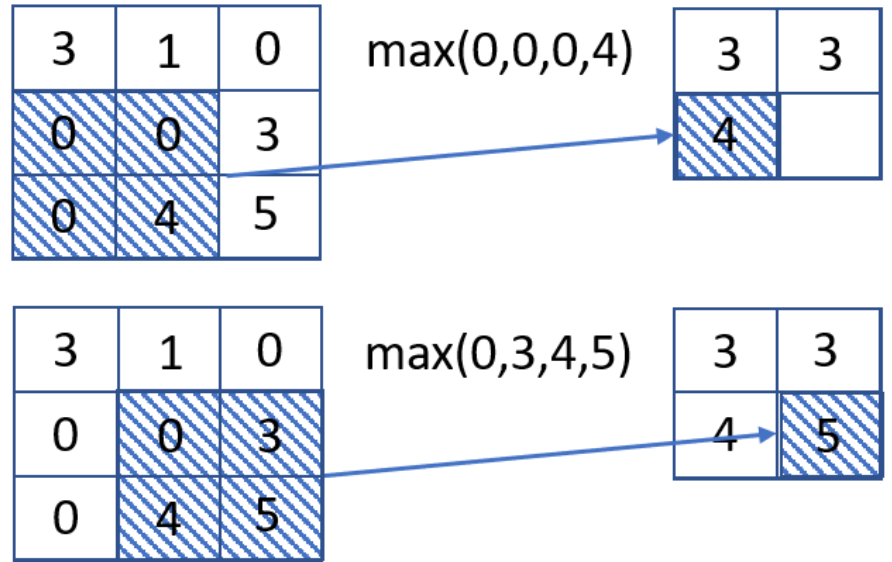

Fonte: Próprio autor.

\subsubsection{Camadas totalmente conectadas (Fully Connected)}

As camadas totalmente conectadas (fully connected) também são utilizadas em uma rede neural clássica. Normalmente, são utilizadas no final da rede, para se obter a saída da rede convolucional. O tamanho da última camada que é equivalente à quantidade de neurônios na camada, será a quantidade de classes possíveis que a saída pode apresentar. [SOCIEDADE BRASILEIRA DE COMPUTAÇÃO, 2017]

Após a última camada de fully connected é possível colocar a camada de Softmax que transforma as saídas para valores entre 0 e 1, cuja soma de todas as saídas é 1 . Com essa transformação cada classe apresentaria um valor que corresponderia à probabilidade daquela amostra pertencer a dada classe. [SOCIEDADE BRASILEIRA DE COMPUTAÇÃO, 2017] 


\section{$\underline{3.7 .4 \text { AlexNet }}$}

Alex e seus colaboradores (2012) treinaram uma rede neural convolucional para um subconjunto de dados da base ImageNet usado nas competições ILSVRC-2010 e ILSVRC-201. Nessas competições foi usado um subconjunto de 1000 categorias dentre as 22000 da ImageNet, que contém mais 15 milhões de imagens em alta resolução categorizada em diversos tipos (por exemplos veículos, frutas, animais e etc,).

A rede AlexNet propôs o uso de novos procedimentos que melhoraram o desempenho e reduziram o tempo de treinamento. Um desses procedimentos é a Normalização da Resposta Local e outro é o Pooling com sobreposição. Também foram utilizadas técnicas efetivas de prevenção de sobreajuste como o descarte (dropout) de entradas. Além disso foi usada uma função de saída para os neurônios cujo o tempo de treinamento com gradiente decrescente é mais rápido, conforme descrito na próxima seção.

\subsubsection{Camadas de neurônios retificadores (ReLUs)}

A rede AlexNet usa neurônios denominados ReLUs, definidos por Nair e Hinton (2010). Esses neurônios relacionam a entrada $x$ à saída por intermédio da função $f(x)$ $=\max (0, x)$, diferentemente dos modelos padrão cuja saída é dada pelas funções hiperbólica ou logística, apresentadas na seção 3.6.1. (Krizhevsky; Sutskever; Hinton, 2012)

\subsubsection{Camadas de Normalização}

Os neurônios de ReLUs apresentam a característica desejada de não necessitarem da normalização da entrada. Uma entrada positiva garante que o neurônio seja ativo e influencie a rede. Uma entrada negativa inativa o neurônio.

Em relação às saídas da camada de neurônios ReLUs é interessante realizar a normalização local, porque se obtém mais facilmente a generalização da rede AlexNet. 
A atividade da resposta normalizada $b_{x, y}^{i}$ é dada pela expressão

$$
b_{x, y}^{i}=a_{x, y}^{i} /\left(2+10^{-4} \sum_{j=\max (0, i-2)}^{\min (N-1, i+2)}\left(a_{x, y}^{j}\right)^{2}\right)^{0,75},
$$

em que $a_{x, y}^{i}$ é a atividade do neurônio calculada ao se aplicar o kernel $i$ na posição $(x, y), N$ é o número total de kernels na camada, e o somatório em $j$ é calculado sobre 5 mapas de kernels. A ordem dos mapas de kernels é arbitrária e determinada antes do início do treinamento. (Krizhevsky; Sutskever; Hinton, 2012)

\subsubsection{Camadas de Pooling com sobreposição}

Sendo $z \times z 0$ tamanho da unidade de pooling e $s$ o espaçamento entre os centros das unidades de pooling, se temos $s<z$ ocorre sobreposição, o que não ocorreria se $s=$ Z.

Alex e colaboradores utilizaram $\mathrm{s}=2$ e $\mathrm{z}=3$, e observaram que durante o treinamento de modelos com pooling, era mais difícil haver sobreajuste com sobreposição do que sem sobreposição.

\subsubsection{Camadas de descarte (dropout)}

Uma camada de descarte (dropout) é constituída pela mesma quantidade de neurônios da camada anterior. A entrada de cada neurônio dessa camada de descarte é igual à saída do respectivo neurônio da camada anterior. Por outro lado, a saída de cada neurônio dessa camada de descarte pode ser igual a sua entrada ou igual a zero, com probabilidade de $50 \%$. Os neurônios cujas saídas são zeradas não participariam no treinamento, reduzindo as complexas adaptações em conjunto dos neurônios. Com isso, os neurônios das camadas posteriores seriam estimulados a aprender parâmetros mais robustos, a partir de subconjuntos aleatórios de neurônios, em vez de depender de um determinado neurônio específico. (Krizhevsky; Sutskever; Hinton, 2012)

No período de classificação, são utilizados todos os neurônios com suas saídas multiplicadas por 0,5 . 
O descarte seria usado nas duas primeiras camadas totalmente conectadas. Sem o descarte, a rede apresentaria um substancial sobreajuste. Com o descarte, no entanto, o número de iterações necessárias para convergir é aproximadamente dobrado.

\subsubsection{Arquitetura}

A arquitetura da rede de AlexNet pode ser vista na figura 25.

As primeiras camadas da rede de AlexNet são formadas por duas sequências de camada convolucional, ReLU, normalização da resposta e Max-pooling.

A primeira camada da rede é uma camada convolucional que filtra a imagem de

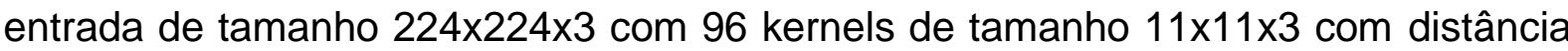
entre os centros de 4 pixels.

A quinta camada da rede é uma camada convolucional com 256 kernels de tamanho $5 \times 5 \times 48$.

Após as oito camadas das duas sequências iniciais, há três sequências contendo apenas as camadas de convolução e ReLU. Nessas três sequências não há nenhuma camada de normalização ou pooling.

$\mathrm{Na}$ nona camada da rede, que é uma camada convolucional, há 384 kernels de tamanho $3 \times 3 \times 256$.

Na décima primeira camada da rede, que também é uma camada convolucional, há 384 kernels de tamanho $3 \times 3 \times 192$.

E na décima terceira camada há 256 kernels de tamanho 3×3×192.

Após a décima quarta camada da rede, que é uma camada ReLU, também há uma camada de Max-pooling.

Posteriormente há duas sequências de camadas totalmente conectadas, ReLU e dropout.

A penúltima camada da rede é totalmente conectada e a última camada do AlexNet é do tipo softmax e tem 1000 neurônios cujas saídas correspondem as 1000 classes ou 
categorias. O neurônio com maior valor da saída indica que a imagem é classificada como pertencente à essa categoria específica.

Figura 25: Arquitetura da rede de AlexNet

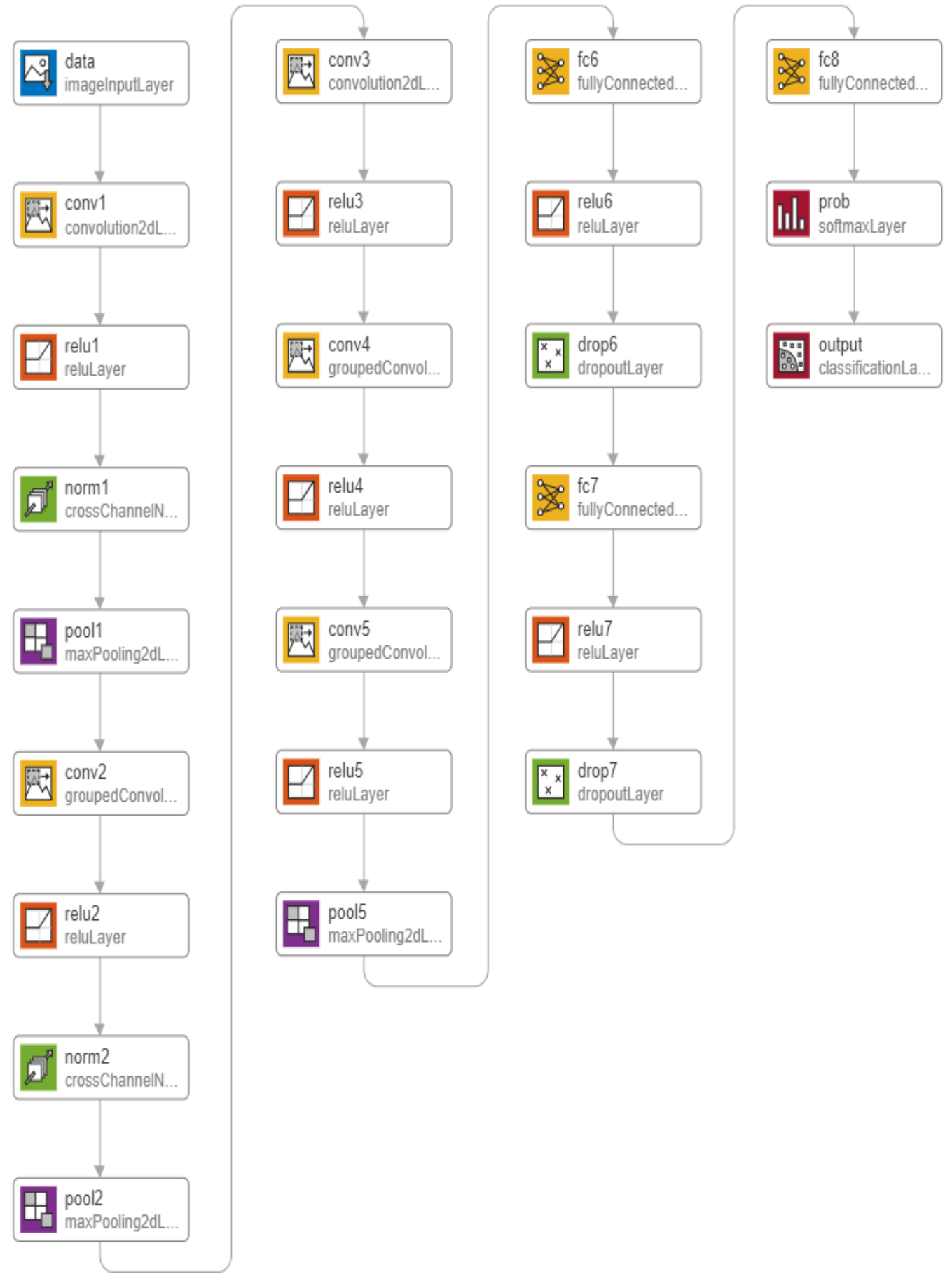

Fonte: Próprio autor. 


\section{RESULTADOS}

Este capítulo apresenta os resultados para diversos cenários. Esses resultados permitem avaliar a influência da base de dados, do método de treinamento na classificação do discriminante linear. Também permitem analisar a influência da normalização, da duração dos segmentos analisados, do uso de largura de núcleo única e da escolha de parâmetros no desempenho da máquina de vetor de suporte. Por último, permitem comparar o desempenho da máquina de vetor de suporte, da rede neural artificial do tipo alimentação direta e da rede neural convolucional.

\subsection{Comparação de bancos de dados}

A escolha do banco de dados utilizado no treinamento pode influenciar no desempenho dos classificadores. Um classificador treinado com um banco de dados com pouca variabilidade pode não ser capaz de classificar corretamente uma nova amostra com características bem diferentes das usadas no treinamento.

Para verificar qual o melhor banco de dados a ser utilizado, foram realizadas classificações com o classificador de discriminante linear (DL) utilizando os 60 parâmetros sugeridos por Trardi e seus colaboradores (2018). Para cada banco de dados foi realizado o treinamento com o método de leave-one-subject-out. Para cada amostra de teste, as amostras dos demais indivíduos do mesmo banco de dados foram usadas para treinamento. Os resultados obtidos para este banco de dados podem ser vistos na tabela 2 .

Tabela 2: Acurácia (ACU), sensibilidade (SEN) e especificidade (ESP) para as bases de dados MIT-BIH e Long-Term com treinamento pelo método leave-one-subject-out obtidas com o classificador DL.

\begin{tabular}{|c|c|c|c|c|}
\hline Teste & Treinamento & ACU (\%) & SEN (\%) & ESP (\%) \\
\hline MIT-BIH & MIT-BIH & 97,10 & 98,04 & 96,33 \\
\hline Long-Term & Long-Term & 99,47 & 99,59 & 99,25 \\
\hline
\end{tabular}

Como pode ser visto na tabela 2, as taxas de acurácia, sensibilidade e especificidade foram 2,37\%, 1,55\% e 2,92\% menores para o MIT-BIH Atrial Fibrillation do que as 
obtidas para o banco de dados Long-Term Atrial Fibrillation. Isto mostra que há diferença nas taxas obtidas ao se utilizar bancos de dados diferentes.

Ao realizar o treinamento utilizando todas amostras de um banco de dados para classificar o outro banco de dados foram obtidos os seguintes resultados que podem ser vistos na tabela 3

Tabela 3: Acurácia (ACU), sensibilidade (SEN) e especificidade (ESP) para as bases de dados MIT-BIH e Long-Term utilizando as amostras do outro banco de dados para treinamento obtidas com o classificador DL.

\begin{tabular}{|c|c|c|c|c|}
\hline Teste & Treinamento & ACU (\%) & SEN (\%) & ESP (\%) \\
\hline MIT-BIH & Long-Term & 84,49 & 99,14 & 72,46 \\
\hline Long-Term & MIT-BIH & 88,24 & 99,89 & 67,95 \\
\hline
\end{tabular}

Para cada banco de dados, a maior acurácia é obtida ao realizar a classificação com o mesmo banco para treinamento. $E$ ao realizar o treinamento com outro banco de dados há uma redução de até $12,61 \%$ na acurácia, 1,10\% na sensibilidade e 31,30\% na especificidade.

$\mathrm{Na}$ literatura, as maiores acurácias foram obtidas realizando o treinamento e a classificação com o mesmo banco de dados Long-Term Atrial Fibrillation. Portanto, para se fazer uma comparação com a literatura, os próximos resultados serão baseados nesse mesmo banco de dados e comparados aos valores obtidos nesta seção.

\subsection{Influência do método de treinamento no desempenho do discriminante linear}

Uma pergunta a ser respondida é se a escolha das amostras de treinamento e teste fazem diferença nas taxas obtidas, apesar de serem do mesmo banco de dados. Por isso foram realizadas classificações com o mesmo classificador DL e os 60 parâmetros sugeridos por Trardi e seus colaboradores (2018) utilizados na seção 4.1. A diferença estaria nos conjuntos de treinamento e teste, em que foram realizados 50 sorteios aleatórios dos conjuntos de treinamento e teste. Para cada conjunto sorteado, 
metade das amostras de cada indivíduo do banco de dados Long-Term Atrial Fibrillation foram utilizadas para treinamento e outra metade para teste.

Este resultado foi comparado com o obtido na seção 4.1, em que foi aplicado o método leave-one-subject-out. Para cada amostra de teste, as amostras dos demais indivíduos foram usadas para treinamento. Desta forma, as amostras do mesmo indivíduo não foram utilizadas para treinamento e teste.

A tabela 4 mostra os resultados para o treinamento pelo método leave-one-subjectout e a média e o desvio-padrão dos resultados para o treinamento por sorteios aleatórios.

Tabela 4: Acurácia (ACU), sensibilidade (SEN) e especificidade (ESP) para treinamento pelos métodos de sorteios e de remoção do indivíduo a ser testado (leave-one-subject-out) obtidas com o classificador DL.

\begin{tabular}{|c|c|c|c|}
\hline Treinamento & ACU (\%) & SEN (\%) & ESP (\%) \\
\hline Sorteios & $99,61 \pm 0,04$ & $99,63 \pm 0,05$ & $99,57 \pm 0,07$ \\
\hline Leave-one-subject-out & 99,47 & 99,59 & 99,25 \\
\hline
\end{tabular}

Como pode ser visto na tabela 4, em média a acurácia, sensibilidade e especificidade obtidas com os sorteios são superiores às obtidos com o método de leave-onesubject-out. Para escolhas aleatórias, o melhor caso (aquele com as maiores taxas) resultou em acurácia, sensibilidade e especificidade de 99,70\%;99,74\%; e 99,62\% respectivamente. O melhor caso obtido apresentou um aumento de até 0,14\%;0,04\% e $0,32 \%$ na acurácia, sensibilidade e especificidade respectivamente em relação ao método de leave-one-subject-out.

Ao realizar sorteios aleatórios é possível utilizar amostras do mesmo indivíduo tanto para treinamento como para teste. Como as características das amostras de um mesmo indivíduo são mais próximos do que entre indivíduos diferentes, é possível selecionar conjuntos de treinamento e teste que apresentem amostras semelhantes em ambos os conjuntos. Essa semelhança garante melhor desempenho do que na classificação de amostras de teste que não tem semelhança com as amostras de treinamento. 
Mas como a diferença dos resultados é pequena e numa situação prática não é possível realizar treinamento com amostras do indivíduo que se deseja realizar a classificação, o método de leave-one-subject-out é o mais adequado. Portanto o método de treinamento de leave-one-subject-out será utilizado nas próximas seções.

\subsection{Comparação entre o discriminante linear e a máquina de vetor de suporte}

Após determinar o melhor banco de dados e o método de treinamento a ser utilizado, outra questão analisada foi o melhor classificador. Cada classificador tem as suas características com limitações e vantagens em relação a outros classificadores. Um classificador pode ter um desempenho superior a outros para um certo tipo de dados e parâmetros utilizados e um desempenho inferior para outro tipo de dados e parâmetros. Usualmente um classificador mais robusto permite taxas de classificações superior, mas exige um tempo de processamento maior.

Nessa dissertação foram comparados inicialmente os classificadores DL e a máquina de vetor de suporte (SVM, Support Vector Machine). Para comparação foram utilizados os mesmos 60 parâmetros sugeridos por Trardi e seus colaboradores (2018) e o método de treinamento de leave-one-subject-out utilizados nas seções anteriores com o banco de dados Long-Term Atrial Fibrillation.

Os resultados apresentados pelo discriminante linear e pela máquina de vetor de suporte sem normalização podem ser vistos na tabela 5 .

Tabela 5: Acurácia (ACU), sensibilidade (SEN) e especificidade (ESP) obtidas com o classificador DL e SVM para o treinamento pelo método de leave-one-subject-out.

\begin{tabular}{|c|c|c|c|}
\hline Classificador & ACU (\%) & SEN (\%) & ESP (\%) \\
\hline Linear & 99,47 & 99,59 & 99,25 \\
\hline SVM & 99,64 & 99,60 & 99,70 \\
\hline
\end{tabular}

O DL apresenta a limitação de não conseguir classificar corretamente os dados que não são linearmente separáveis. A acurácia de $99,47 \%$ obtida com o DL mostra que a maioria dos dados são linearmente separáveis. A SVM conseguiu classificar 
corretamente apenas 60 trechos a mais do que o DL, dentre os 37525 trechos analisados.

\subsection{Influência da normalização no desempenho da máquina de vetor de suporte}

Os cálculos do DL incluem uma normalização dos parâmetros de entrada. Por isso, foi feita uma verificação se a normalização dos parâmetros pode influenciar no desempenho do classificador SVM utilizando os mesmos 60 parâmetros da seção 4.3. A tabela 6 mostra os resultados obtidos ao realizar a normalização dos 60 parâmetros de entrada, usando o método leave-one-subject-out de treinamento da SVM.

Tabela 6: Acurácia (ACU), sensibilidade (SEN) e especificidade (ESP) na classificação com o treinamento pelo método de leave-one-subject-out e com SVM, com o uso ou não de normalização dos parâmetros.

\begin{tabular}{|c|c|c|c|}
\hline Normalização & ACU (\%) & SEN (\%) & ESP (\%) \\
\hline SVM sem normalização & 99,64 & 99,60 & 99,70 \\
\hline SVM com normalização & 99,82 & 99,92 & 99,66 \\
\hline
\end{tabular}

Neste caso houve um aumento das taxas de acurácia $(+0,18 \%)$ e de sensibilidade $(+0,32 \%)$ e uma pequena redução da especificidade $(-0,04 \%)$ ao realizar a normalização.

A normalização altera o desempenho da máquina de vetor de suporte, porque dá a mesma importância para os parâmetros que antes eram menosprezados, em função de sua menor amplitude e variabilidade. Quando todos os parâmetros foram normalizados, a detecção dos casos de fibrilação atrial foi facilitada resultando na alta sensibilidade. Por outro lado, alguns poucos casos que antes eram considerados corretamente como normais passaram a ser considerados erroneamente como trechos com fibrilação atrial. Comparando os resultados do DL com os resultados da SVM com a normalização, há um aumento de 0,35\%; 0,33\% e 0,41\% de acurácia, sensibilidade e especificidade respectivamente. Esse resultado mostra que a SVM com a normalização apresenta um desempenho melhor na classificação da fibrilação atrial, por isso ela será utilizada nas próximas seções. 


\subsection{Determinação de uma largura de núcleo única}

Numa situação prática não é possível realizar o ajuste da largura de núcleo (sigma) utilizado na SVM para cada indivíduo que se deseja realizar a classificação. Por isso, seria necessário utilizar um único valor da largura de núcleo. Para se obter a melhor largura de núcleo a ser utilizada, foi levantada a curva da acurácia em função da largura sigma. Para isso foram utilizados os mesmos 60 parâmetros da seção $4.4 \mathrm{com}$ normalização e com treinamento pelo método de leave-one-subject-out. A figura 26 mostra a curva da acurácia obtida para o banco de dados Long-Term Atrial Fibrillation.

Figura 26: Acurácia obtida por sigma utilizado para banco de dados Long-Term com os parâmetros normalizados e com treinamento pelo método de leave-one-subject-

out

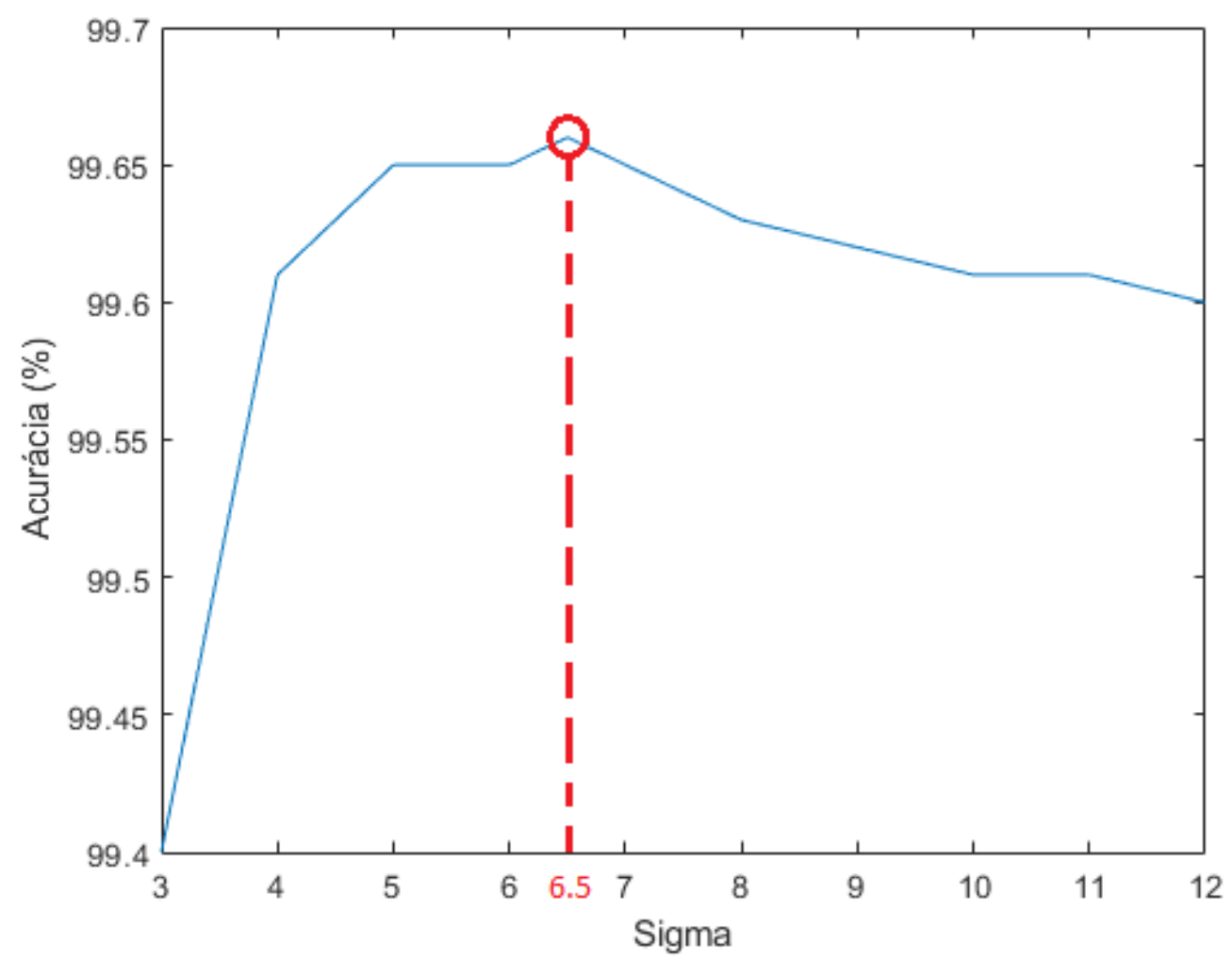

Fonte: Próprio autor.

A máxima acurácia $(99,66 \%)$ foi obtida para a largura de núcleo sigma igual a 6,5. Para este valor de sigma são obtidas $99,65 \%$ de sensibilidade e $99,67 \%$ de especificidade. 
Tabela 7: Acurácia (ACU), sensibilidade (SEN) e especificidade (ESP) na classificação com os parâmetros normalizados e com treinamento pelo método de leave-one-subject-out com SVM, com o uso ou não de sigma único.

\begin{tabular}{|c|c|c|c|}
\hline Sigma & ACU (\%) & SEN (\%) & ESP (\%) \\
\hline SVM com sigma variável & 99,82 & 99,92 & 99,66 \\
\hline SVM com sigma único & 99,66 & 99,65 & 99,67 \\
\hline
\end{tabular}

Para cada conjunto de treinamento há uma largura de núcleo ótima. Utilizando uma largura de núcleo única, alguns conjuntos que antes eram classificados corretamente ao utilizar a largura de núcleo ótima poderiam passar a ser classificados erroneamente. Isso resultaria na redução da acurácia em relação à apresentada na tabela 6 de 0,16\%. Apesar dessa redução, a acurácia fornecida pela SVM é superior àquela fornecida pelo classificador linear de 0,19\%.

Por esse método ser mais semelhante a uma situação prática e a acurácia obtida ainda ser superior àquela obtida com o classificador linear, o treinamento com sigma único será utilizado nas seções posteriores.

\subsection{Influência do tamanho dos segmentos}

Além das altas taxas de classificações, também é desejado obter o resultado no menor intervalo de tempo possível. Como o tempo de processamento da SVM é maior que o tempo de processamento do DL, também foram analisadas algumas formas de diminuir esse tempo. A diminuição do tamanho dos segmentos seria uma dessas formas. Quanto menor o tamanho dos segmentos menor seria o tempo necessário para captar o sinal e o tempo para calcular os parâmetros. Para analisar a influência do tamanho do segmento, cada segmento de 60 ciclos do banco de dados Long-Term Atrial Fibrillation foi dividido em dois segmentos de 30 ciclos. Esse procedimento resultou em 70550 segmentos de 30 ciclos, sendo 44818 o número de segmentos com FA e 25732 o número de normais.

Os parâmetros foram recalculados para os segmentos de 30 ciclos. Foi realizada a normalização dos parâmetros no treinamento e posteriormente a classificação foi realizada utilizando sigma único de 6,5. O treinamento da SVM foi realizado pelo método de treinamento leave-one-subject-out. A tabela 8 mostra as taxas obtidas. 
Tabela 8: Acurácia (ACU), sensibilidade (SEN) e especificidade (ESP) na classificação com os parâmetros normalizados e com treinamento pelo método de leave-one-subject-out para SVM com sigma único e número de ciclos variável.

\begin{tabular}{|c|c|c|c|}
\hline Quantidade de ciclos & ACU (\%) & SEN (\%) & ESP (\%) \\
\hline 60 & 99,66 & 99,65 & 99,67 \\
\hline 30 & 99,32 & 99,41 & 99,16 \\
\hline
\end{tabular}

Este resultado mostra que, ao reduzir o tamanho do segmento estudado, também há diminuição das taxas obtidas. Neste caso houve redução de 0,34\% na acurácia, de $0,14 \%$ na sensibilidade e de $0,51 \%$ na especificidade. Portanto é possível escolher o tamanho do segmento de acordo com as necessidades. Como nesse trabalho se almeja obter as melhores taxas de classificação, sem a preocupação de processamento de tempo real serão utilizados 60 ciclos por segmento nas próximas seções.

\subsection{Redução dos números de parâmetros}

Além da redução do tamanho do segmento, outro método de diminuir o tempo de processamento seria reduzir o número de parâmetros utilizados.

A redução analisada foi a eliminação dos parâmetros obtidos pelos sinais diferenciados ou de dinâmica de ordem maior, dentre os 60 parâmetros sugeridos por Trardi e colaboradores (2018). Esse procedimento resultou na redução em 15 parâmetros. Também se verificou que o parâmetro $S D S D_{i}$ apresentava valores semelhantes aos de $S D_{i}$ e de $S D 2_{i}$ e, por isso, esses últimos parâmetros $\left(S D_{i}\right.$ e $\left.S D 2_{i}\right)$ também foram descartados.

Os 13 parâmetros remanescentes dentre os propostos por Trardi e seus colaboradores (2018) foram complementados com o parâmetro razão entre o máximo e o mínimo intervalo RR. Essa inclusão resultou em um novo conjunto de 14 parâmetros para classificação.

A tabela 9 mostra as taxas obtidas ao realizar o treinamento da SVM com o método de treinamento leave-one-subject-out, com sigma único, com novo conjunto de 14 parâmetros em comparação com as obtidas com 60 parâmetros. 
Tabela 9: Acurácia (ACU), sensibilidade (SEN) e especificidade (ESP) para classificações com 60 e 14 parâmetros normalizados como entrada para SVM com sigma único e método de treinamento leave-one-subject-out.

\begin{tabular}{|c|c|c|c|}
\hline Parâmetros & $\mathrm{ACU}(\%)$ & SEN (\%) & ESP (\%) \\
\hline 60 & 99,66 & 99,65 & 99,67 \\
\hline 14 & 99,73 & 99,67 & 99,83 \\
\hline
\end{tabular}

Com os 14 parâmetros foi possível obter taxas de acurácias superiores aos 60 sugeridos por Trardi e colaboradores, o que indicaria que é possível obter taxas elevadas com um baixo número de parâmetros.

\subsection{Seleção dos parâmetros}

Para se obter um conjunto de parâmetros que apresentava a melhor taxa de classificação com a menor quantidade de parâmetros possíveis, seria necessário verificar mais parâmetros além dos sugeridos por Trardi e colaboradores (2018).

Cada parâmetro da revisão da literatura foi calculado para o banco de dados LongTerm Atrial Fibrillation e fornecido separadamente como entradas para o classificador linear. O treinamento foi feito pelo método leave-one-subject-out. Os parâmetros foram calculados somente para os intervalos $R R$, mas não para as suas derivadas.

As tabelas 10 e 11 mostram as acurácias na classificação pelo discriminante linear de cada parâmetro isoladamente. A tabela 10 mostra os 21 parâmetros que fornecem acurácia superior a $90 \%$ e a tabela 11 os demais, que fornecem acurácia inferior a $90 \%$. 
Tabela 10: Classificação com o discriminante linear e treinamento pelo método leave-one-subject-out dos parâmetros cujas acurácias são superiores a $90 \%$.

\begin{tabular}{ccc}
\hline Autor & Parâmetro & Acurácia $(\%)$ \\
\hline Proposto pelo autor & FC & 98,02 \\
\hline Adaptado de Trardi & FuzzyEn & 97,70 \\
\hline Adaptado de Trardi & SampEn & 97,54 \\
\hline Adaptado de Trardi & SENP & 97,34 \\
\hline Trardi & VAI1 & 97,30 \\
\hline Trardi & FuzzyEn1 & 96,70 \\
\hline Gilani & Dist04 & 96,57 \\
\hline Adaptado de Rizwan & MMR & 96,22 \\
\hline Trardi & QSE1 & 95,98 \\
\hline Adaptado de Trardi & COSEn & 95,86 \\
\hline Kennedy & COSEn & 95,78 \\
\hline Kennedy & CV & 94,62 \\
\hline Mohebbi e Ghassemian & PCSD1 & 94,18 \\
\hline Trardi & SDSD1 & 94,18 \\
\hline Dash & RMSSD & 94,13 \\
\hline Nuryani & SDSD & 94,13 \\
\hline Trardi & SD1 & 94,13 \\
\hline Outros & ShanEn & 93,07 \\
\hline Logan e Healey & VAR & 92,53 \\
\hline Kennedy & RMSSD & 92,41 \\
\hline Adaptado de Wrobel & MAD & 91,11 \\
\hline Mohebbi e Ghassemian & PCSD2 & 91,00 \\
\hline & &
\end{tabular}


Tabela 11: Classificação com o discriminante linear e treinamento pelo método leave-one-subject-out dos parâmetros cujas acurácias são inferiores a 90\%.

\begin{tabular}{ccc}
\hline Autor & Parâmetro & Acurácia (\%) \\
\hline Trardi & COSEn & 87,80 \\
\hline Trardi & SC1 & 73,82 \\
\hline Meza & QIr & 70,38 \\
\hline Meza & MdIr & 69,01 \\
\hline Dash & TPR & 67,04 \\
\hline Gilani & MV & 67,02 \\
\hline Trardi & MV1 & 67,02 \\
\hline Gilani & SDSDW & 66,83 \\
\hline Trardi & RMS1 & 65,34 \\
\hline Meza & DPIr & 63,65 \\
\hline Marsili & ShanEn & 63,53 \\
\hline Trardi & VLI1 & 62,19 \\
\hline Trardi & SampEn1 & 60,55 \\
\hline Trardi & KC1 & 55,03 \\
\hline Mohebbi e Ghassemian & SampEn & 53,68 \\
\hline Slocum & REB & 52,83 \\
\hline Nuryani & MVDRR & 51,18 \\
\hline Gilani & CSDW & 42,09 \\
\hline Trardi & ApEn1 & 37,71 \\
\hline & &
\end{tabular}

Ao aplicar a técnica de análise de componentes principais com os parâmetros apresentados nas tabelas 10 e 11 notou-se que, a partir de 7 componentes principais, era possível representar $100 \%$ da variabilidade. Esse resultado indica que seria suficiente usar 10 parâmetros para se obter acurácia elevada. O gráfico da variabilidade pelo número de componentes pode ser visto na figura 27. 
Figura 27: Variabilidade por número de componentes principais: de $98,44 \%$ para 1 componente até $100,00 \%$ para 7 ou mais componentes.

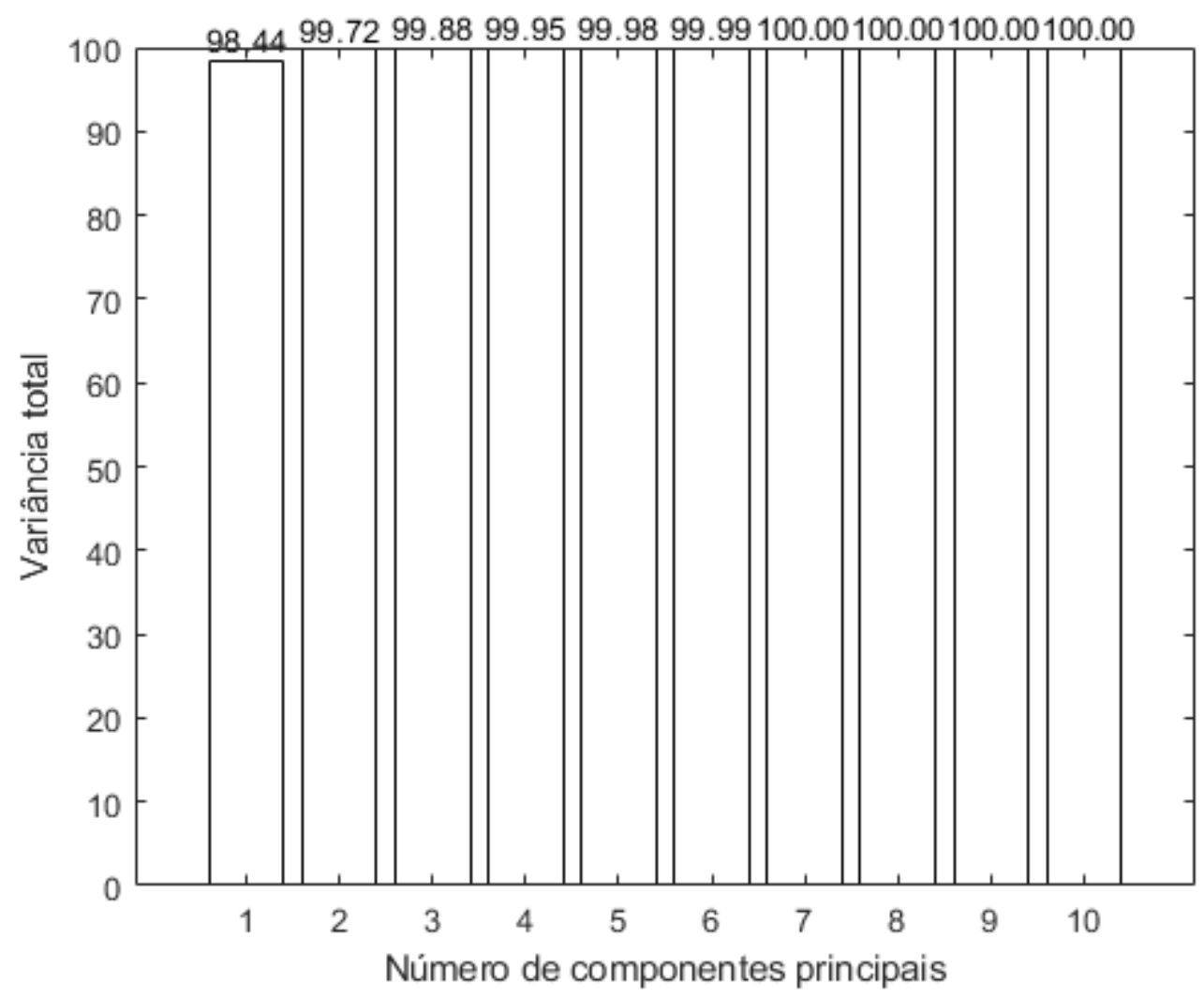

Fonte: Próprio autor.

Primeiramente, os parâmetros que apresentaram taxas inferiores a $90 \%$ foram descartados. Em seguida, dentre os demais parâmetros, quando havia dois ou mais parâmetros semelhantes, só se manteve um deles. Por fim, restaram 14 parâmetros apresentadas na tabela 12: a soma das magnitudes dos componentes espectrais dividido pela média dos intervalos RR (FC); a média dos valores absolutos dos ângulos (VAl) e a média dos senos do gráfico de Poincaré (SENP); a soma das distâncias menores que 0,04 dos pontos à origem do gráfico de Lorenz (Dist04); a razão entre o máximo intervalo RR e o mínimo intervalo RR (MMR); o coeficiente de variação (CV); a mediana dos desvios absolutos entre os intervalos $\mathrm{RR}$ e o valor médio (MAD); a variância (VAR); o desvio padrão do valor absoluto das diferenças (PCSD1); as entropias amostral (SampEn), de Fuzzy (FuzzyEn) e de Shannon (ShanEn); o coeficiente da entropia amostral (COSEn) e a entropia amostral quadrática (QSE). 
Tabela 12: Classificação com o discriminante linear e treinamento pelo método leave-one-subject-out dos 14 parâmetros resultantes da eliminação dos parâmetros semelhantes ou iguais da tabela 10.

\begin{tabular}{cccc}
\hline Índice & Autor & Parâmetro & Acurácia (\%) \\
\hline 1 & Proposto pelo autor & FC & 98,02 \\
\hline 2 & Adaptado de Trardi & FuzzyEn & 97,70 \\
\hline 3 & Adaptado de Trardi & SampEn & 97,54 \\
\hline 4 & Adaptado de Trardi & SENP & 97,34 \\
\hline 5 & Trardi & VAI1 & 97,30 \\
\hline 6 & Gilani & Dist04 & 96,57 \\
\hline 7 & Adaptado de Rizwan & MMR & 96,22 \\
\hline 8 & Trardi & QSE & 95,98 \\
\hline 9 & Adaptado de Trardi & COSEn & 95,86 \\
\hline 10 & Kennedy & CV & 94,62 \\
\hline 11 & Mohebbi e & PCSD1 & 94,18 \\
\hline 12 & Ghassemian & ShanEn & 93,07 \\
\hline 13 & Adaptado de Zhang & VAR & 92,53 \\
\hline 14 & Logan e Healey & MAD & 91,11 \\
\hline
\end{tabular}

A partir dos 14 parâmetros restantes, foram realizadas combinações entre os parâmetros formando conjuntos de 2 a 14 parâmetros. Cada conjunto foi utilizado como parâmetros de entrada do classificador linear, utilizando o método de treinamento leave-one-subject-out.

A figura 28 mostra as melhores acurácias obtidas para cada quantidade de parâmetros utilizada para formar os conjuntos e os parâmetros utilizados em cada conjunto podem ser vistas na tabela 13. Os índices dos parâmetros utilizados na tabela 13 são referentes aos índices mostrados na tabela 12. 
Figura 28: Acurácia por número de parâmetros como entrada no classificador linear

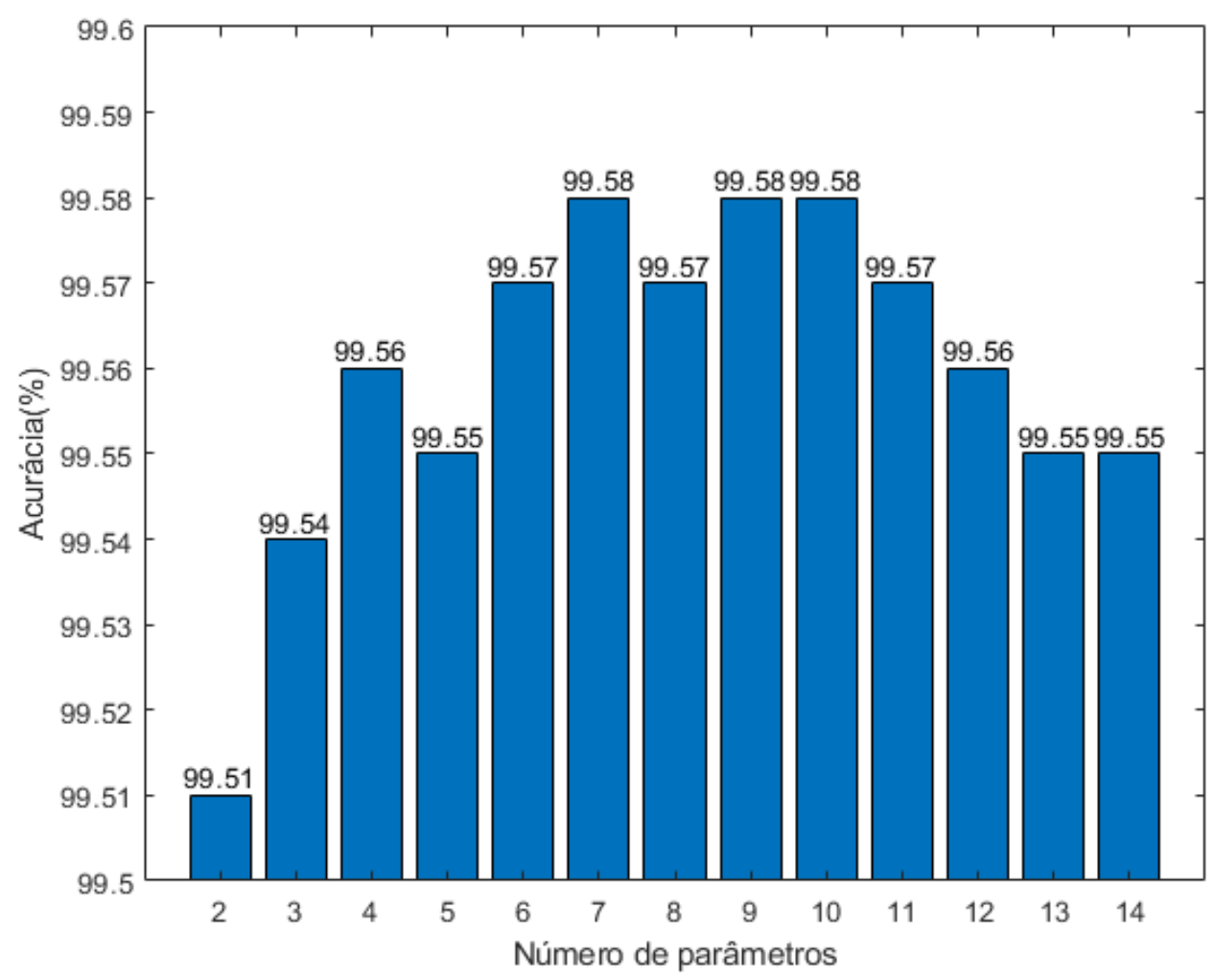

Fonte: Próprio autor.

Tabela 13: Classificação com o discriminante linear dos conjuntos de 1 a 14 parâmetros formados a partir dos parâmetros mostrados na tabela 12 .

\begin{tabular}{ccc}
\hline Número de parâmetros & Índice dos Parâmetros & Acurácia $(\%)$ \\
\hline 2 & 9 e 11 & 99,51 \\
\hline 3 & 7,9 e 11 & 99,54 \\
\hline 4 & $7,8,9$ e 11 & 99,56 \\
\hline 5 & $7,8,9,11$ e 14 & 99,55 \\
\hline 6 & $2,3,4,5,9$ e 11 & 99,57 \\
\hline 7 & $2,3,4,5,9,11$ e 14 & 99,58 \\
\hline 8 & $2,3,4,5,8,9,11$ e 14 & 99,57 \\
\hline 9 & $2,3,4,5,8,9,11,12$ e 14 & 99,58 \\
\hline 10 & $2,3,4,5,8,9,11,12,13$ e 14 & 99,58 \\
\hline 11 & $1,2,3,4,5,7,9,10,11,12$ e 14 & 99,57 \\
\hline 12 & $1,2,3,4,5,7,8,9,10,11,12$ e 14 & 99,56 \\
\hline 13 & $1,2,3,4,5,7,8,9,10,11,12,13$ e 14 & 99,55 \\
\hline 14 & todos & 99,55 \\
\hline
\end{tabular}

Os resultados apresentados na figura 27 e tabela 13 mostram que há uma elevação da acurácia com um aumento da quantidade de parâmetros utilizados até um patamar da faixa de 7 a 10 parâmetros com a acurácia de 99,58\%. A partir dos 11 parâmetros 
há uma redução da acurácia. É também possível destacar que assim como na figura 26 em que a partir dos 7 componentes era possível representar $100 \%$ da variabilidade, a partir dos conjuntos de 7 parâmetros é possível obter a acurácia máxima de 99,58\%.

Os conjuntos de 7 a 10 parâmetros que apresentaram as melhores acurácias também foram utilizados como entrada para SVM realizando o treinamento pelo método de leave-one-subject-out com sigma único. As acurácias obtidas por número de parâmetros utilizados podem ser vistas na figura 29 a seguir.

Figura 29: Acurácia por número de parâmetros como entrada no classificador SVM

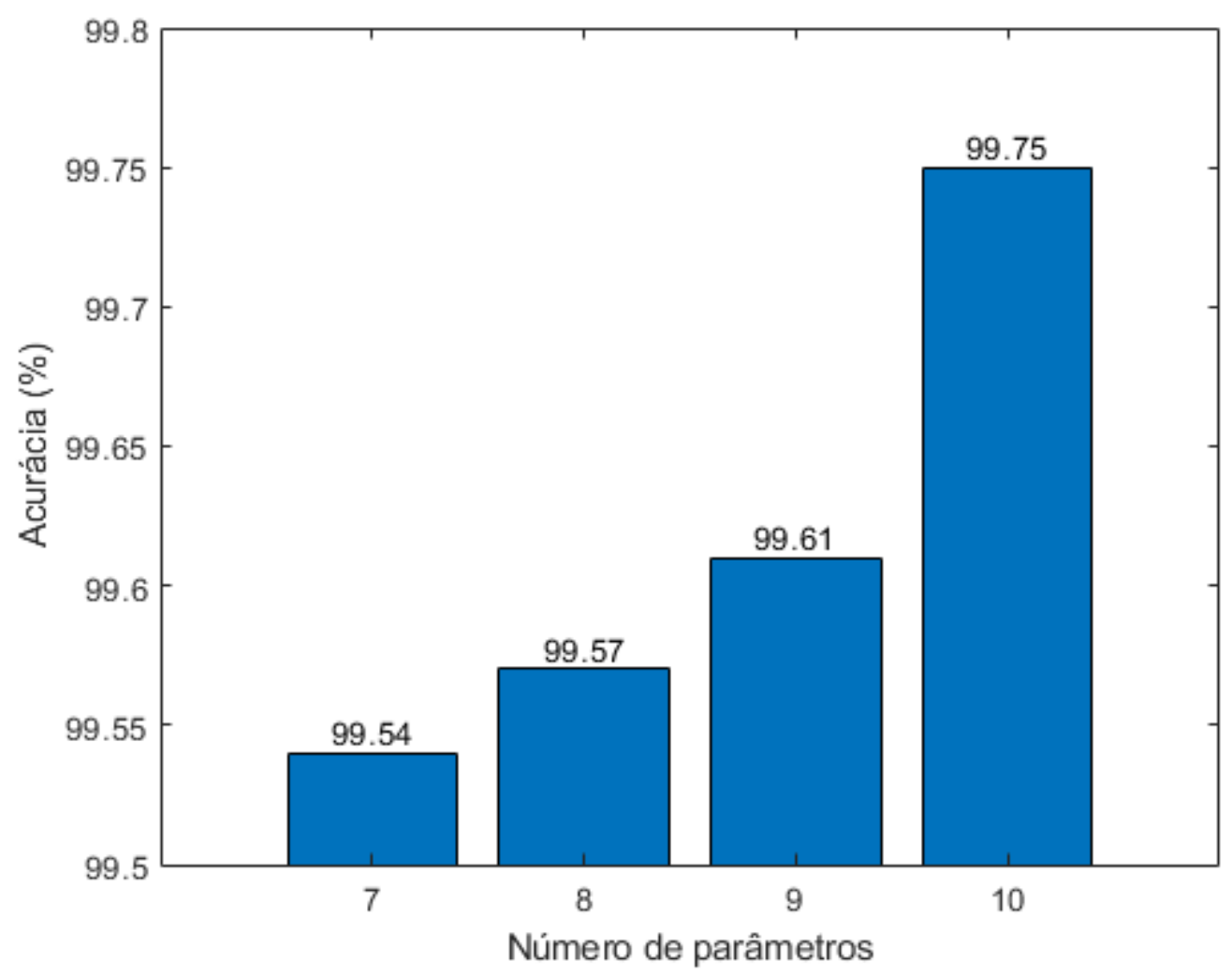

Fonte: Próprio autor.

A tabela 14 mostra as taxas obtidas utilizando a SVM com o novo conjunto de 10 parâmetros em comparação com as obtidas com o conjunto de 60 parâmetros propostos por Trardi e colaboradores (2018) e com o conjunto de 14 parâmetros propostos na seção 4.7. 
Tabela 14: Acurácia (ACU), sensibilidade (SEN) e especificidade (ESP) para diversos conjuntos de parâmetros normalizados como entrada para SVM com o treinamento pelo método leave-one-subject-out.

\begin{tabular}{|c|c|c|c|}
\hline Parâmetros & ACU (\%) & SEN (\%) & ESP (\%) \\
\hline 60 parâmetros sugeridos por Trardi & 99,66 & 99,65 & 99,67 \\
\hline 14 & 99,73 & 99,67 & 99,83 \\
\hline 10 & 99,75 & 99,65 & 99,94 \\
\hline
\end{tabular}

Os resultados da tabela 14 mostram que é possível reduzir a quantidade de parâmetros e obter taxas de classificação superiores. Com 60 parâmetros obtiveramse as piores taxas. A maior acurácia foi obtida com 10 parâmetros, em que houve aumento de $0,09 \%$ e $0,27 \%$ de especificidade em comparação com 60 parâmetros.

O conjunto de parâmetros que apresentou a maior acurácia seria formado por: a média dos valores absolutos dos ângulos (VAl) e a média dos senos do gráfico de Poincaré (SENP); a mediana dos desvios absolutos entre os intervalos $R R$ e o valor médio (MAD); a variância (VAR); o desvio padrão do valor absoluto das diferenças (PCSD1); as entropias amostral (SampEn), de Fuzzy (FuzzyEn) e de Shannon (ShanEn); o coeficiente da entropia amostral (COSEn) e a entropia amostral quadrática (QSE).

Para efeitos de comparação com a literatura, também foi realizada a classificação com o treinamento pelo método de sorteio utilizando o mesmo conjunto de 10 parâmetros. A Tabela 15 mostra as taxas obtidas para os dois métodos de treinamento.

Tabela 15: Acurácia (ACU), sensibilidade (SEN) e especificidade (ESP) para o conjunto de 10 parâmetros normalizados como entrada para SVM com os dois métodos de treinamento.

\begin{tabular}{|c|c|c|c|}
\hline Treinamento & $\mathrm{ACU}(\%)$ & $\mathrm{SEN}(\%)$ & $\mathrm{ESP}(\%)$ \\
\hline Leave-one-subject-out & 99,75 & 99,65 & 99,94 \\
\hline Sorteio & 99,81 & 99,81 & 99,81 \\
\hline
\end{tabular}




\subsection{Rede Neural Artificial do tipo alimentação direta}

O conjunto de 10 parâmetros obtidos na seção 4.8 também foi utilizado como entrada para a rede neural artificial do tipo alimentação direta. A rede implementada apresentava duas camadas internas e uma camada de saída. Por ser uma rede neural do tipo alimentação direta, as entradas dos neurônios de cada camada estariam ligadas às saídas dos neurônios da camada anterior.

Foram analisados os dois métodos de treinamento, o método que deixa os sinais de um indivíduo reservados para o teste e os demais são usados no treinamento (leaveone-subject-out) e o método de sorteio da metade das amostras para o treinamento e a outra metade para o teste. Como a rede neural artificial necessita de um conjunto de validação, para cada conjunto de treinamento 15\% das amostras foram separadas aleatoriamente para serem utilizados como validação.

Considerando o método de treinamento leave-one-subject-out, a maior acurácia foi obtida quando havia 21 neurônios em cada camada interna. Para o caso do método de sorteio, a maior acurácia foi obtida quando havia 19 neurônios em cada camada interna.

A tabela 16 mostra os resultados obtidos para a rede neural com o método de treinamento leave-one-subject-out e de sorteios para 19 e 21 neurônios nas camadas internas.

Tabela 16: Acurácia (ACU) para treinamentos pelos métodos de sorteios e de remoção do indivíduo a ser testado (leave-one-subject-out) para 19 e 21 neurônios nas camadas internas.

\begin{tabular}{|l|l|l|}
\hline Número de neurônios & Método de treinamento & Acurácia (\%) \\
\hline 19 & Sorteio & 99,88 \\
\hline 19 & leave-one-subject-out & 99,46 \\
\hline 21 & Sorteio & 99,85 \\
\hline 21 & leave-one-subject-out & 99,70 \\
\hline
\end{tabular}

O resultado mostrado na tabela 16 indica que nesse classificador (rede neural de alimentação direta) há uma maior influência do conjunto de treinamento utilizado, havendo uma variação de até $0,42 \%$ enquanto na SVM houve uma variação de apenas $0,06 \%$. 


\subsection{Rede Neural Convolucional}

Também foi implementada a rede neural convolucional AlexNet e as imagens de Poincaré foram utilizadas como entrada da rede. A paciência da validação foi utilizada como critério de parada.

Para se obter o melhor valor da paciência da validação foram realizados treinamentos pelo método de sorteio com valores de paciência da validação variando de 1 até 70 (60).

A figura 30 mostra o gráfico da acurácia pela paciência da validação. Para valores baixos da paciência da validação há uma variação grande da taxa de acurácia obtida, indicando que a rede estava realizando a parada do treinamento num mínimo local que estava distante do mínimo global desejado.

A partir da paciência da validação de valor 25 houve uma estabilização do valor da acurácia obtida, com o seu maior valor (99,85\%) na paciência da validação 57 .

A tabela 17 mostra os resultados obtidos para os métodos de treinamento leave-onesubject-out e de sorteio para a rede neural convolucional. 
Figura 30: Gráfico da acurácia pela paciência da validação da rede neural convolucional treinada com o método de leave-one-subject-out com a imagem de Poincaré como entrada.

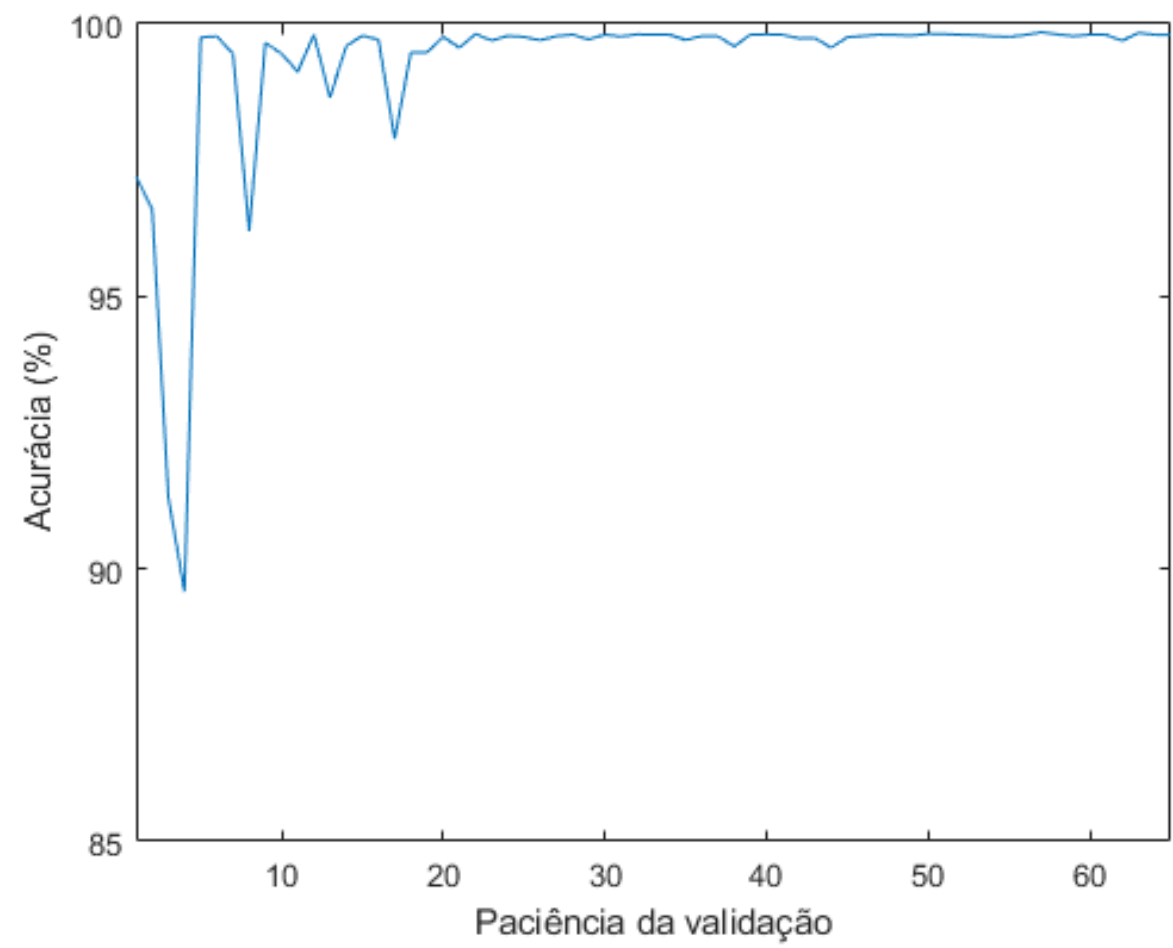

Fonte: Próprio autor.

Tabela 17: Acurácia (ACU) para treinamentos pelos métodos de sorteios e leaveone-subject-out para rede neural convolucional para paciência de validação igual a

\begin{tabular}{|c|c|}
\hline \multicolumn{2}{|c|}{57.} \\
\hline Método de treinamento & Acurácia (\%) \\
\hline Sorteio & $99,85 \%$ \\
\hline Leave-one-subject-out & $99,57 \%$ \\
\hline
\end{tabular}

A acurácia obtida com a rede neural convolucional foi semelhante à obtida com a rede neural artificial do tipo alimentação direta, usando-se o treinamento pelo método de sorteio. 


\section{DISCUSSÃO}

\subsection{Comparação entre banco de dados}

Os resultados mostram que as taxas mudam com o uso de banco de dados diferentes. Neste caso a acurácia obtida para o banco de dados MIT-BIH Atrial Fibrillation foi 2,92\% inferior à obtida para o banco de dados Long-Term Atrial Fibrillation.

Cada banco de dados pode apresentar sinais de qualidades (relações sinal-ruído) diferentes. Além disso, a precisão das marcações também pode ser diferente. Esses fatos podem influenciar o grau de dificuldade da classificação. Outro fator que pode influenciar a classificação é a prevalência de sinais de fibrilação atrial em comparação aos normais. Também pode haver tipos diferentes de fibrilação atrial: paroxística, persistente ou permanente dependendo da base de dados. Dependendo da distribuição de sinais normais e com os diversos tipos de fibrilação atrial, alguns parâmetros poderiam diferenciar bem as classes de um dado banco de dados, enquanto outros parâmetros poderiam diferenciar melhor as classes do outro banco de dados.

Um banco de dados que apresente pouca variabilidade dos seus dados pode ter a separação mais fácil das classes diferentes quando comparado a um banco com alta variabilidade. Mas provavelmente haveria mais dificuldades em classificar corretamente um novo sinal que apresentasse características diferentes das utilizadas no treinamento. Nessa dissertação, ao realizar o treinamento com um banco de dados e a classificação com outro banco de dados, houve uma redução de 8,86\% a 12,61\% na acurácia. Isso indica que a base de treinamento deve ser obtida no mesmo hospital ou clínica em que o classificador será aplicado, para evitar essa pronunciada diminuição da acurácia.

\subsection{Influência do método de treinamento}

Os resultados mostram que há uma pequena diminuição das taxas obtidas ao realizar o treinamento pelo método leave-one-subject-out em vez do método de sorteios. Este resultado era esperado já que dados de um mesmo indivíduo apresentam 
características semelhantes e isto facilitaria a classificação. Ao sortear metade dos dados para treinamento e metade para teste, os dados do mesmo indivíduo estarão tanto no treinamento como no teste, o que não ocorre ao utilizar o método de leaveone-subject-out em que os dados de todos os demais indivíduos seriam utilizados para treinar o classificador de um dado indivíduo.

$\mathrm{Na}$ aplicação prática, os sinais de um mesmo indivíduo não são utilizados para treinamento e posterior detecção dos novos sinais do indivíduo. Em vez disso, os dados coletados anteriormente de outros indivíduos são usados para detectar um novo indivíduo. Assim, o ideal seria a aplicação do método leave-one-subject-out para o treinamento do classificador, pois esse método se assemelha mais à situação prática.

A redução de $0,14 \%$ da acurácia ao se alterar o método de treinamento é bem menor que os $12,61 \%$ que ocorre ao se realizar o treinamento com o outro o banco de dados. Indicando que as características de um indivíduo influenciam menos do que as características gerais de um banco de dados, sendo indicado padronizar as condições de captação dos sinais.

\subsection{Influência da normalização no desempenho da máquina de vetor de suporte}

Na máquina de vetor de suporte, o hiperplano de separação é obtido pela maior distância entre os vetores de suporte ao hiperplano. Por isso, os parâmetros que apresentam as maiores amplitudes e variabilidades são priorizados, já que estes têm maior influência no valor da distância. A normalização altera o desempenho da máquina de vetor de suporte, porque dá a mesma importância para os parâmetros que antes eram menosprezados, em função de sua menor amplitude e variabilidade. Neste caso, ao serem realizadas as normalizações, houve um aumento das taxas obtidas de até $0,18 \%$ em relação à classificação sem normalizações. Por isso, concluise que a normalização dos parâmetros deve ser sempre aplicada antes da classificação, visando melhorar o desempenho da SVM.

\subsection{Determinação de uma largura de núcleo única}

No caso em que sejam realizadas classificações de novos dados provenientes de outros indivíduos, não seria viável realizar uma otimização da largura do núcleo 
(sigma) para cada indivíduo, como realizado no método de treinamento leave-onesubject-out. Por isso, seria necessário utilizar uma largura de núcleo predeterminada que apresentasse a melhor quantidade de acertos possíveis. Ao levantar a curva da acurácia pela largura de núcleo, verificou-se que houve um pico para sigma igual a 6,5, com acurácia de 99,66\%.

Ao se utilizar essa única largura de núcleo $(6,5)$, na classificação dos parâmetros normalizados de todos os indivíduos, houve uma diminuição de 0,16\% da acurácia. Esse resultado era esperado porque, para alguns indivíduos, o valor da largura de núcleo estaria afastado do valor ótimo. Essa redução de apenas 0,16\% na acurácia indica que as excelentes taxas de classificação não foram obtidas graças a um eventual sobre-ajuste (overfitting) e que houve uma influência muito pequena da otimização do valor da largura de núcleo para cada conjunto de treinamento. Isso indica que o classificador treinado com o núcleo único pode apresentar altas taxas de classificações de novos dados de outros indivíduos. Esse resultado é promissor já que um classificador treinado com uma base de dados adquiridos num hospital ou clínica poderia ser usado para detectar fibrilação atrial em novos pacientes desse mesmo hospital ou clínica.

\subsection{Influência do tamanho dos segmentos}

A redução da duração dos segmentos está associada à redução do número de ciclos analisados. Portanto, diminui a quantidade de memória necessária e o tempo de processamento dos parâmetros.

Mas, por outro lado, essa redução do número de ciclos analisados resulta na queda do desempenho. A redução da duração para 50\% resultou numa redução de $0,34 \%$ na acurácia da SVM. Esse resultado mostra que é viável diminuir o tamanho do segmento e o tempo necessário para se obter a classificação sem que haja grande perda no desempenho.

\subsection{Redução dos números de parâmetros}

Os resultados obtidos mostram que, com a redução da quantidade de parâmetros, é possível obter taxas melhores. Analisando os parâmetros propostos por Trardi e seus colaboradores (2018), verificou-se que alguns parâmetros tinham características ou até mesmo valores semelhantes, como no caso do SD (o desvio padrão dos valores 
absolutos das diferenças entre RR consecutivos sobre raiz de dois), que apresentava valores semelhantes a SDSD (desvio padrão das diferenças entre RR consecutivos). Eliminando estes parâmetros, foi possível reduzir a quantidade de parâmetros e se obteve uma taxa de classificação superior. Esse fato mostra que nem sempre usar uma quantidade maior de parâmetros resulta em taxas superiores.

\subsection{Seleção dos parâmetros}

Outra forma de se obter um conjunto melhor de parâmetros foi por meio da escolha entre os parâmetros com as melhores acurácias separadamente, desconsiderando os parâmetros que forneceram acurácias inferiores a 90\%.

Foi possível obter uma acurácia superior escolhendo um número menor de parâmetros que individualmente conseguiam representar melhor a características da fibrilação atrial.

Isso mostra que a escolha dos parâmetros é um processo importante, pois uma boa escolha permite selecionar um conjunto menor de parâmetros que apresentam as maiores taxas de classificação. Além disso, com a redução do número de parâmetros calculados, há também redução da memória necessária e do tempo para processamento.

\subsection{Comparação entre os classificadores}

\subsubsection{Comparação entre DL e SVM}

Ao analisar as taxas obtidas com o DL e do classificador SVM sem normalização, é possível verificar que a SVM apresentou acurácia 0,17\% maior que o DL. Como o DL consegue separar de forma ótima apenas as classes que são linearmente separáveis,

é justificável que a SVM apresentasse melhor desempenho na classificação da fibrilação atrial, já que a SVM consegue classificar inclusive as classes linearmente não separáveis.

A aplicação de uma normalização aos parâmetros de entrada da SVM causa um aumento na acurácia. Nesse caso, o aumento da acurácia em relação ao DL atinge $0,35 \%$. 


\subsubsection{Comparação entre SVM e rede neural artificial de alimentação direta}

A acurácia obtida com a SVM ao se realizar o treinamento por sorteio é 0,07\% menor que a fornecida pela rede neural. Mas, por outro lado, a acurácia obtida com a SVM para o treinamento pelo método leave-one-subject-out é $0,05 \%$ maior que a obtida com a rede neural.

Esse resultado indica que houve um sobre-ajuste aos dados utilizados no treinamento, resultando em melhor classificação dos dados que apresentam características semelhantes (do mesmo indivíduo) e maior dificuldade na classificação dos dados de características diferentes (de indivíduos diferentes).

Para realizar o treinamento pelo método de leave-one-subject-out foram necessários em média 5,394 s para a máquina de vetor de suporte, enquanto para a rede neural foram necessários em média 11,7735 s por indivíduo. Para ambos os classificadores foi necessário menos do que 100 us para classificar um novo segmento de 60 ciclos.

Como na prática são realizados treinamentos e classificações com indivíduos diferentes, a SVM seria o classificador mais adequado, porque o tempo de treinamento e classificação da máquina de vetor de suporte é menor do que a rede neural artificial.

\subsubsection{Comparação da rede neural artificial do tipo alimentação direta com a rede}

\section{convolucional}

Ao realizar o método de treinamento por sorteio, a rede neural convolucional apresentou acurácia semelhante ao da rede neural artificial do tipo alimentação direta.

A vantagem da rede neural convolucional em comparação com a rede neural clássica está na possibilidade de se obter taxas semelhantes, mas sem a necessidade de cálculo prévio dos parâmetros.

Apesar de haver o passo de transformação do sinal de ECG ou do tacograma para uma imagem, esse processamento é mais rápido e simples do que os cálculos dos parâmetros utilizados como entrada para outros classificadores.

Para obtenção dos 10 parâmetros de um segmento foi necessário em média 7 ms, enquanto que para gerar e salvar as imagens foi necessário em média de $3 \mathrm{~ms}$. Por outro lado, o tempo necessário para realizar a classificação e treinamento foram 
superiores aqueles das redes neurais básicas e da máquina de vetor de suporte. Em média foram necessários $2 \mathrm{~ms}$ para classificação e aproximadamente $2 \mathrm{~h}$ para treinamento.

O treinamento da rede neural convolucional exige um tempo muito superior comparado aos dos outros classificadores, mas o tempo necessário para obter a classificação de um segmento com o classificador já treinado é menor comparado aos outros classificadores ( $5 \mathrm{~ms}$ para rede convolucional e $7 \mathrm{~ms}$ para máquina de vetor de suporte e rede neural).

Do mesmo modo que a rede neural artificial clássica, na rede convolucional há uma grande influência do conjunto de treinamento utilizado nas taxas de classificação. Ao realizar o método de treinamento leave-one-subject-out, houve uma diminuição pronunciada da acurácia quando comparada àquela da SVM.

\subsection{Comparação com a literatura}

$\mathrm{Na}$ literatura, as maiores acurácias foram obtidas ao realizar a classificação com o treinamento por sorteios. Para que fosse realizada a comparação utilizando os mesmos critérios, as taxas de classificação para os dez parâmetros selecionados foram obtidas utilizando o treinamento por um sorteio de $50 \%$ dos casos para conjunto de treinamento e os demais para o conjunto de teste. A tabela 18 mostra as taxas de classificações fornecidas na literatura em comparação com as melhores taxas obtidas nesse trabalho.

A primeira taxa apresentada foi obtida por Trardi e seus colaboradores (2018) utilizando os 60 parâmetros apresentados na seção 3.2.12.

A maior taxa de classificação obtida na literatura (Trardi e seus colaboradores, 2018b) foi obtida realizando o cálculo de 72 parâmetros e a seleção dos melhores 14 parâmetros, utilizando o método de aprendizado por múltiplos kernels.

Por último estão as taxas obtidas com os 10 parâmetros obtidos a partir da seleção do melhor conjunto de parâmetros dentro dos 41 descritos na seção 3.2 e seu processo na seção 4.8. Para a rede neural convolucional foram utilizadas as imagens de Poincaré descritas na seção 3.2.14. 
Tabela 18: Acurácia (ACU) fornecida na literatura em comparação com a melhor taxa obtida por sorteio dos conjuntos de treinamento e teste.

\begin{tabular}{|c|c|c|}
\hline Parâmetros & Classificador & ACU (\%) \\
\hline 60 (Trardi e seus colaboradores, 2018) & SVM & 98,22 \\
\hline $72 \rightarrow 14$ (Trardi e seus colaboradores, 2018b) & SVM & 99,77 \\
\hline $41 \rightarrow 10$ (Este trabalho) & SVM & 99,81 \\
\hline Imagens de Poincaré & CNN & 99,85 \\
\hline $41 \rightarrow 10$ (Este trabalho) & RNA & 99,88 \\
\hline
\end{tabular}

A tabela 18 mostra que esse trabalhou apresentou acurácias levemente superiores aos fornecidos na literatura. Essa acurácia foi obtida com uma quantidade de parâmetros inferior aos utilizados na literatura.

Nesta dissertação, a escolha dos melhores parâmetros foi realizada de modo mais simples, selecionando o melhor conjunto de 10 parâmetros dos 14 que apresentaram as maiores acurácias individuais. Por meio deste método mais simples, foi possível selecionar um conjunto de parâmetros menores, mas que apresentaram uma acurácia superior às apresentadas na literatura. Esse resultado mostra que não é necessário um método sofisticado de seleção de parâmetros. 


\section{CONCLUSÃO}

Para efeitos de comparação com a literatura utilizou-se o banco de dados Long-Term Atrial Fibrillation e o método de treinamento por sorteio, que consiste em separar metade das amostras para o treinamento e a outra metade para a classificação. Nesta dissertação, obtiveram-se acurácias de 99,81\% (SVM), 99,85\% (rede neural convolucional) e $99,88 \%$ (rede neural clássica), que foram superiores às registradas na literatura de $99,77 \%$ (Trardi et al, 2018b).

Usando o método de treinamento por sorteio, a rede neural do tipo feedforward foi o classificador que apresentou a maior acurácia. Apesar de ser necessária a escolha e o cálculo de parâmetros, a convergência no treinamento foi mais rápida do que aquela da rede convolucional.

No entanto, no método de treinamento por sorteio são utilizados dados dos mesmos indivíduos tanto para treinamento quanto para teste. Isso induz a sobre-ajuste das redes neurais que acabam apresentando piores resultados na classificação de novos dados de outros indivíduos.

Para evitar este sobre-ajuste, classificam-se os dados de um indivíduo (subject) usando um classificador treinado com os dados de demais indivíduos. Esse método de treinamento é denominado leave-one-subject-out e é mais adequado para aplicação prática.

Com alteração do método de treinamento para leave-one-subject-out, a máquina de vetor de suporte se mostrou mais robusta do que as redes neurais, fornecendo a maior acurácia.

Esta dissertação propõe que se utilizem a máquina de vetor de suporte e os seguintes parâmetros normalizados para a detecção da fibrilação atrial: VAl (a média dos valores absolutos dos ângulos de Poincaré), VAR (a variância dos intervalos RR), SENP (média dos senos do gráfico de Poincaré), PCSD1 (desvio padrão do valor absoluto das diferenças dos intervalos RR), MAD (a mediana dos desvios absolutos entre os intervalos RR e o seu valor médio), SampEn (entropia amostral dos intervalos RR), FuzzyEn (entropia de Fuzzy dos intervalos RR), ShanEn (entropia de Shannon dos 
intervalos RR), COSEn (coeficiente da entropia amostral dos intervalos RR) e QSE (entropia amostral quadrática dos intervalos RR). 


\section{REFERÊNCIAS}

Andersson, O.; Rodrigues, J. N. A $400 \mathrm{mV}$ atrial fibrillation detector with 0.56 pJ/operation in 65nm CMOS. In: 2015 IEEE International Symposium on Circuits and Systems (ICAS), 2015, Lisbon, Portugal. Proceedings of ICAS 2015. doi: 10.1109/ISCAS.2015.7169225.

Andersson, O. et al. A 290mV Sub-Vt ASIC for Real-Time Atrial Fibrillation Detection. IEEE Transactions on Biomedical Circuits and Systems, v. 9, n. 3, p. 377-386, June 2015. doi: 10.1109/TBCAS.2014.2354054.

Bumgarner, J. M. et al. Smartwatch Algorithm for Automated Detection of Atrial Fibrillation. Journal of the American College of Cardiology, v. 71, n. 21, p. 23812388, 2018. doi: 10.1016/j.jacc.2018.03.003

Cees de Vos, B. et al. Progression from paroxysmal to persistent atrial fibrillation: clinical correlates and prognosis. Journal of the American College of Cardiology, v. 55, n. 8, p. 725-731, 23 Feb. 2010.

Censi, F. et al. P-wave variability and atrial fibrillation. Scientific Reports, v. 6, n. 1, 26 May 2016. doi: 10.1038/srep26799.

Chong, J. W. et al. Arrhythmia discrimination using a smart phone. IEEE Journal of Biomedical and Health Informatics, v. 19, n. 3, p. 815-824, May 2015. doi: 10.1109/JBHI.2015.2418195.

Chong, J. W. et al. Motion and noise artifact-resilient atrial fibrillation detection algorithm for a smartphone. IN: 2016 IEEE-EMBS International Conference on Biomedical and Health Informatics (BHI), 2016, Las Vegas, NV. Proceedings of BHI 2016, p. 591-594. doi: 10.1109/BHI.2016.7455967.

Cristianini, N.; Taylor, J. S. An Introduction to Support Vector Machines and other Kernel-Based Learning Methods. Cambridge, Cambridge University Press, 2000.

Dash S. et al. Automatic real time detection of atrial fibrillation. Annals of Biomedical Engineering, v. 37, n.9, p.1701-1709, Sept. 2009. doi: 10.1007/s10439-009-9740-z.

De Moraes E. R. F. L. et al. Prevalence of atrial fibrillation and stroke assessment based on telemedicine screening tools in a primary healthcare setting. European Journal of Internal Medicine, v. 67, p.36-41, 2019. doi: 10.1016/j.ejim.2019.04.024.

Drew C. A.; Wiersma Y. F.; Huettmann F. Predictive Species and Habitat Modeling in Landscape Ecology. 1st ed. Springer-Verlag New York, 2011.

Fine T.L. Feedforward Neural Network Methodology. 1st ed. Springer-Verlag New York, 1999. 
Fukunaga K. Introduction to Statistical Pattern Recognition. 2nd ed. Boston: Academic Press, 1990.

García M. et al. Application of the relative wavelet energy to heart rate independent detection of atrial fibrillation. Computers in Biology and Medicine, v. 131, p. 157-168 2016. doi: 10.1016/j.cmpb.2016.04.009

Gilani M.; Eklund J. M.; Makrehchi M. Automated detection of atrial fibrillation episode using novel heart rate variability features. In: Annual International Conference of the Engineering in Medicine and Biology Society (EMBC), 38, 2016, Proceedings of the $38^{\text {th }}$ Engineering in Medicine and Biology Conference, IEEE, Orlando, FL, USA. Doi: 10.1109/EMBC.2016.7591473

Golderberger A. et al. PhysioBank, PhysioToolkit, and PhysioNet: components of a new research resource for complex physiologic signals. Circulation, vol. 101, n.23, p.215-220, 2000.

Gonzalez R. C.; Woods R. E.; Eddins S. L. Digital Image Processing Using MATLAB. Upper Saddle River, Prentice Hall, 2004.

Gonzalez R. C.; Woods R. E. Processamento Digital de Imagens. 3a ed., São Paulo, Pearson Prentice Hall, 2010.

Goodfellow I.; Bengio Y.; Courville A. Deep Learning. MIT Press, 2016. Disponível em <http://www.deeplearningbook.org" http://www.deeplearningbook.org>. Acesso em 08 jan. 2021.

Guo J. M. et al. Atrial fibrillation Detection in Spectrogram Based on Convolutional Neural Networks. IN: 2019 International Symposium on Intelligent Signal Processing and Communication Systems (ISPACS), 2019, Proceeding of 2019 International Symposium on Intelligent Signal Processing and Communication Systems (ISPACS), Taipei, Taiwan.

Guyton A. C.; Hall J. E. Tratado de Fisiologia Médica. 12a ed., Rio de Janeiro: Elsevier, 2011.

Hargittai S. Is it possible to detect atrial fibrillation by simply using RR intervals? In: Computing in Cardiology Conference (CinC), 2014, Proceedings of CinC 2014, IEEE, Cambridge, MA, USA.

Haykin S. Redes neurais: princípios e prática; tradução de Paulo Martins Engel. 2a ed., Porto Alegre: Bookman, 2001.

Haykin S. Neural Networks and Learning Machines. 3rd ed. New York, N.Y. Toronto: Pearson Education inc., 2009.

Hecht-Nielsen R. Theory of the backpropagation neural network. In: International 1989 Joint Conference on Neural Networks, 1989, Proceeding of International 1989 Joint Conference on Neural Networks, v.1, p. 593-605, 1989, Washington, DC, USA, doi: 10.1109/IJCNN.1989.118638. 
Hurnanen T. et al. Automated detection of atrial fibrillation based on time-frequency analysis of seismocardiograms. IEEE Journal of Biomedical and Health Informatics, v. 21, n. 5, p. 1233-1241, 2017. Doi: 10.1109/JBHI.2016.2621887.

Huxley R. R. et al. Absolute and attributable risks of atrial fibrillation in relation to optimal and borderline risk factors. The atherosclerosis risk in communities (ARIC) study, Circulation, v. 123, n.14, p.1501-1508, Apr. 2011. Doi: 10.1161/CIRCULATIONAHA.110.009035.

Islam, M. S. et al. Rhythm-based heartbeat duration normalization for atrial fibrillation detection. Computers in Biology and Medicine, v. 72, p.160-169, 2016.

Jalali A.; Lee M. Atrial Fibrillation Prediction With Residual Network Using Sensitivity and Orthogonality Constraints. IEEE Journal of Biomedical and Health Informatics, v. 24, n. 2, Feb. 2020.

Jolliffe I. T. Principal Component Analysis. 2.ed, New York: Springer, 2002.

Kalsi M.; Prakash N. R. A new algorithm for detection of atrial fibrillation. In: International Conference on Electrical, Electronics, and Optimization Techniques (ICEEOT), 2016, Proceedings of ICEEOT 2016, IEEE, Chennai, India. Doi: 10.1109/ICEEOT.2016.7755288.

Kannathal N. et al. Cardiac health diagnosis using data fusion of cardiovascular and haemodynamic signals. Computer Methods and Programs in Biomedicine, v. 82, n.2, p.87-96, 2006.

Kelwade J.P.; Salankar S. S. Radial basis function neural network for prediction of cardiac arrhythmias based on heart rate time series. In: IEEE International Conference on Control, Measurement and Instrumentation (CMI), 1, 2016. Proceedings of the $1^{\text {st }}$ Control, Measurements and Instrumentation Conference, IEEE, Koltaka, India, Doi: 10.1109/CMI.2016.7413789.

Kennedy A. et al. The accuracy of beat-interval based algorithms for detecting atrial fibrillation. In: Computing in Cardiology Conference (CinC), 2015, Proceedings of CinC 2015, IEEE, Nice, France. Doi: 10.1109/CIC.2015.7411055.

Kennedy A. et al. Automated detection of atrial fibrillation using R-R intervals and multivariated-based classification. Journal of Electrocardiology, v. 49, n. 6, p. 871876, 2016.

Koivisto T. et al. Automatic detection of atrial fibrillation using MEMS accelerometer. In: Computing in Cardiology Conference (CinC), 2015, Proceedings of CinC 2015, IEEE, Nice, France, Doi: 10.1109/CIC.2015.7411039.

Krizhevsky A.; Sutskever I.; G. E. Hinton. ImageNet Classification with Deep Convolutional Neural Networks, Communications of the ACM. v. 60, n. 6, p. 84-90, 2017. Doi: 10.1145/3065386. ISSN 0001-0782 
Ladavich S.; Ghoraani B. Developing an atrial activity-based algorithm for detection of atrial fibrillation. In: $36^{\text {th }}$ Engineering in Medicine and Biology Conference, 2014, Proceedings of the $36^{\text {th }}$ Engineering in Medicine and Biology Conference, 2014; 2014:54-7, doi: 10.1109/EMBC.2014.6943527

Linker D. T. Accurate automated detection of atrial fibrillation in ambulatory recordings. Cardiovascular Engineering and Technology, v. 7, n. 2, p. 182-189, June 2016. Doi: 10.1007/s13239-016-0256-z.

Logan B.; Healey J. Robust detection of atrial fibrillation for a long term telemonitoring system, Computers in Cardiology, 2005, doi: 10.1109/CIC.2005.1588177.

Ludhwani D.; Wieters J. S. Paroxysmal Atrial Fibrillation. In: StatPearls. Treasure Island (FL): StatPearls Publishing; 2020 Jan.

Mabrouki R.; Khaddoumi B.; Sayadi M. Atrial fibrillation detection on electrocardiogram. In: International Conference on Advanced Technologies for Signal and Image Processing (ATSIP), 2., 2016, Proceedings of the $2^{\text {nd }}$ Advanced Technologies for Signal and Image Processing Conference, IEEE, Monastir, Tunisia, doi: 10.1109/ATSIP.2016.7523112.

Maji U.; Mitra M.; Pal S. Differentiating normal sinus rhythm and atrial fibrillation in ECG signal: A phase rectified signal averaging based approach. In: International Conference on Control, Instrumentation, Energy and Communication (CIEC), 2014, Proceedings of the $1^{\text {st }}$ Conference on Control, Instrumentation, Energy and Communication, IEEE, Calcutta, India.

Maji U. et al. Characterizing cardiac arrythmia by optimized window length based PRSA technique. In: International Conference on Control, Instrumentation, Energy \& Communication $(\mathrm{CIEC}), 2$. 2016. Proceedings of the $2^{\text {nd }}$ Conference on Control, Instrumentation, Energy and Communication, IEEE, Kolkata, India, doi: 10.1109/CIEC.2016.7513743.

Marquardt D. W. An algorithm for least-squares estimation of nonlinear parameters. Journal of the Society for Industrial and Applied Mathematics, v. 11, n.2, p. 431441, 1963. Doi: $10.1137 / 0111030$

Marsili I. A. et al. Optimized algorithms for atrial fibrillation detection by wearable teleholter devices. In: International Smart Cities Conference (ISC2), 2016, Proceedings of ISC2 2016, IEEE, Trento, Italy, doi: 10.1109/ISC2.2016.7580863

Mashrur F. R.; Roy A. D.; Saha D. K. Automatic Identification of Arrhythmia from ECG Using AlexNet Convolutional Neural Network. In: $4^{\text {th }}$ International Conference on Electrical Information and Communication Technology (EICT), 2019 Dez. Khulna, Bangladesh.

Meža M. Preliminary study on detection of atrial fibrillation during oscilometric blood pressure measurement. In: International Convention on Information and Communication Technology, Electronics and Microelectronics (MIPRO), 38., 2015, Proceedings of the $38^{\text {th }}$ International Convention on Information and 
Communication Technology, Electronics and Microelectronics, IEEE, Opatija, Croatia, doi: 10.1109/MIPRO.2015.7160453.

Mohebbi M.; Ghassemian H. Prediction of paroxysmal atrial fibrillation based on nonlinear analysis and spectrum and bispectrum features of the heart variability signal. Computer Methods and Programs in Biomedicine, v. 105, n. 1, p. 40-49, Jan. 2012. Doi: 10.1016/j.cmpb.2010.07.011

Moody G. B; Mark R. G. A new method for detecting atrial fibrillation using R-R intervals. Computers in Cardiology, v. 10, p. 227-230, 1983.

Nair V.; Hinton G. E. Rectified linear units improve restricted Boltzmann machines, In: $27 \mathrm{~h}$ International Conference on Machine Learning, 2010, Proceeding of 27h International Conference on Machine Learning, 2010.

Nemati S. et al. Monitoring and detecting atrial fibrillation using wearable technology. In: Annual International Conference of the Engineering in Medicine and Biology Society (EMBC), 38., 2016, Proceedings of the $3^{\text {th }}$ Engineering in Medicine and Biology Conference, IEEE, Orlando, FL, USA, Doi: 10.1109/EMBC.2016.7591456.

Nesheiwat Z.; Goyal A.; Jagtap M. Atrial Fibrillation (A Fib). In: StatPearls. Treasure Island (FL): StatPearls Publishing; 2020 Jan.

Nuryani N. et al. Atrial fibrillation detection using support vector machine. In: Joint International conference Electric Vehicular Technology and Industrial, Mechanical, Electrical, and Chemical Engineering (ICEVT \& IMECE), 2015, Proceedings of ICEVT\&IMECE 2015, IEEE, Surakarta, Indonesia. Doi: 10.1109/ICEVTIMECE.2015.7496672.

Oppenheim A. V.; Lim J. S. Advanced Topics in Signal Processing. Englewood Cliffs, N.J. Prentice-Hall, 1988.

Pänkäälä M. et al. Detection of atrial fibrillation with seismocardiography. In: Annual International Conference of the Engineering in Medicine and Biology Society (EMBC), 38., 2016, Proceedings of the $38^{\text {th }}$ Engineering in Medicine and Biology Conference, IEEE, Orlando, FL, USA, Doi: 10.1109/EMBC.2016.7591695.

Petrutiu S.; Sahakian A. V.; Swiryn S. Abrupt changes in fibrillatory wave characteristics at the termination of paroxysmal atrial fibrillation in humans. EP Europace, v. 9, n. 7, p. 466-470, 2007

Physionet. Long Term AF Database, 30 out. 2008. Disponível em: < https://physionet.org/content/ltafdb/1.0.0/>. Acesso em: 07 ago. 2021.

Potpara T. S.; Lip G. Y. H. A brief history of "lone" atrial fibrillation: from "a peculiar pulse irregularity" to a modern public health concern, Current Pharmaceutical Design, v. 21, n.5, p. 679-96, 2015.

Pürefellner $\mathrm{H}$. et al. P-wave evidence as a method for improving algorithm to detect atrial fibrillation in insertable cardiac monitors. Heart Rhythm, 2014, v. 11, p.1575-83. 
Rizwan M. et al. AF detection from ECG recordings using feature selection sparse coding, and ensemble learning, Physiological Measurement, v. 39, n.2, p.1-10, 2018.

Shan S. M. et al. Reliable PPG-based algorithm in atrial fibrillation detection. In: IEEE Biomedical Circuits and Systems Conference (BioCAS), 2016, Proceedings of BioCAS 2016, IEEE, Shanghai, China, doi: 10.1109/BioCAS.2016.7833801.

Sheikh S.A. et al. Nonparametric spectral estimation technique to estimate dominant frequency for atrial fibrillation detection. Journal of Signal and Information Processing, v. 6, n.4, Nov. 2015, pp.266-276.

Singh S. et al. Atrial Fibrillation and Premature Contraction Classification Using Convolutional Neural Network. In: International Conference on Intelligent Computing and Control Systems (ICICCS 2019), 2019, Proceedings of the International Conference on Intelligent Computing and Control Systems (ICICCS 2019). 2019 May, Madurai India.

Slocum J.; Sahakian A.; Swiryn S. Diagnosis of atrial fibrillation form surface electrocardiograms based on computed-detected atrial activity, Journal of Electrocardiology, v. 25, n. 1, p.1-8, Jan. 1992.

SOCIEDADE BRASILEIRA DE CARDIOLOGIA. Diretrizes Brasileiras de Fibrilação Atrial. Arquivos Brasileiros de Cardiologia, v. 92, n. 6, supl.1, p.1-39, 2009.

SOCIEDADE BRASILEIRA DE CARDIOLOGIA. Diretrizes Brasileiras de Fibrilação Atrial. Arquivos Brasileiros de Cardiologia, v.106, n.4, supl.2, p.1-22, 2016.

SOCIEDADE BRASILEIRA DE COMPUTAÇÃO. Tópicos em gerenciamento de dados e informações 2017, Brazilian Symposium on Databases - SBBD, Uberlândia: SBC, 2017.

Trardi Y. et al. Multy-Dynamics Analysis of QRS Complex for Atrial Fibrillation Diagnosis, In: $5^{\text {th }}$ International Conference on Control, Decision and Information Technologies (CoDIT), 2018, Proceedings of $5^{\text {th }}$ International Conference on Control, Decision and Information Technologies (CoDIT), Thessaloniki, Greece, April 2018, p.1067-1072

Trardi Y. et al. A novel Method to identify Relevant Features for Automatic Detection of Atrial Fibrillation, In: Mediterranean Conference on Control and Automation (MED), 2018, Proceedings of $\mathbf{2 6}^{\text {th }}$ Mediterranean Conference on Control and Automation (MED), Zadar, Croatia, June 2018, p. 613-618.

Verberk W. J. et al. Screening for atrial fibrillation with automated blood pressure measurement: research evidence and practice recommendations. International Journal of Cardiology, v. 203, p. 465-73, 2016.

Webster J. G. Medical Instrumentation. 3rd ed., New York,1997. 
Wiesel J.; Arsbesfeld B.; Schechter D. Comparison of the Microlife blood pressure monitor with Omron blood pressure monitor for detecting atrial fibrillation. American Journal of Cardiology, v. 114, p.1046-1048, 2014.

Wrobel J. et al. Optimizing the Automated Detection of Atrial Fibrillation Episodes in Long-term Recording Instrumentation. In: International Conference Mixed Design of Integrated Circuits and Systems, 25, 2018. Proceedings of the $25^{\text {th }}$ International Conference Mixed Design of Integrated Circuits and Systems, IEEE, Gdynia, Poland, Doi: 10.23919/MIXDES.2018.8436835.

Xia Y. et al. Detecting atrial fibrillation by deep convolutional neural networks. Computers in Biology and Medicine, v. 93, p. 84-92, Feb. 2018. Doi: 10.1016/j.compbiomed.2017.12.007.

Yoon K. H. et al. Analysis of statistical methods for automatic detection of congestive heart failure and atrial fibrillation with short RR interval time series. In: International Conference on Innovative Mobile and Internet Services in Ubiquitous Computing (IMIS), 9., 2015. Proceedings of the $9^{\text {th }}$ Conference on Innovative Mobile and Internet Services in Ubiquitous Computing, IEEE, Blumenau, Brazil, Doi: $10.1109 /$ IMIS.2015.88

Zhang Y.; Zhang L. Research on Algorithm for Detecting Atrial Fibrillation Using RR Intervals. In: International Conference on Audio, Language and Image Processing (ICALIP), 2018. Proceedings of the ICALIP 2018, IEEE, Shanghai, China, Doi: 10.1109/ICALIP.2018.8455661

Zipes D. P. et al. Braunwald's Heart Disease: a Textbook of Cardiovascular Medicine, 11th ed. Philadelphia, PA: Elsevier, 2018. 


\section{APÊNDICE}

\section{A.1 Coração}

O coração é formado por quatro câmaras e quatro válvulas. As duas câmaras superiores próximas à base do coração são denominadas átrios. As duas câmaras inferiores próximas ao ápice são denominadas ventrículos. $O$ átrio e ventrículo esquerdo são separados pela válvula mitral. As câmaras do lado direito são separadas pela válvula tricúspide. A separação entre o átrio direito e a artéria pulmonar é feita pela válvula pulmonar. $O$ átrio esquerdo e a artéria aorta são separados pela válvula aórtica (Guyton; Hall, 2011).

O coração atua como uma bomba no sistema circulatório. O lado direito é responsável por bombear o sangue para o pulmão e o lado esquerdo é responsável por bombear o sangue para o resto do corpo (Guyton; Hall, 2011).

Para que a circulação do sangue ocorra de forma efetiva, a contração e o relaxamento dos músculos devem ocorrer em ritmo regular. A geração e propagação dos potenciais de ação é feita por um sistema de condução elétrica rápida, ilustrado na Figura 31. A regulação do ritmo cardíaco é coordenada pelo nodo sinusal, situado na parede lateral superior do átrio direito, que gera espontaneamente um potencial de ação. Esse potencial de ação se propaga pelos átrios até atingir o nodo atrioventricular, onde ocorre um atraso na propagação. Em seguida, o potencial de ação se propaga pelos ramos esquerdo e direito de His em direção ao ápice. A propagação pelas fibras de Purkinje segue em direção à base. À medida que o potencial de ação se propaga pelo sistema de condução, o músculo ventricular adjacente também é despolarizado e a propagação se perpetua pelo miocárdio. O retardo na propagação do potencial de ação do átrio para o ventrículo permite que a contração dos átrios e consequente bombeamento do sangue do átrio para o ventrículo ocorram antes da contração dos ventrículos (Guyton; Hall, 2011). 
Figura 31 - Esboço do sistema de condução rápida do coração com nodo sinoatrial, nodo atrioventricular, ramo de His e fibras de Purkinje.

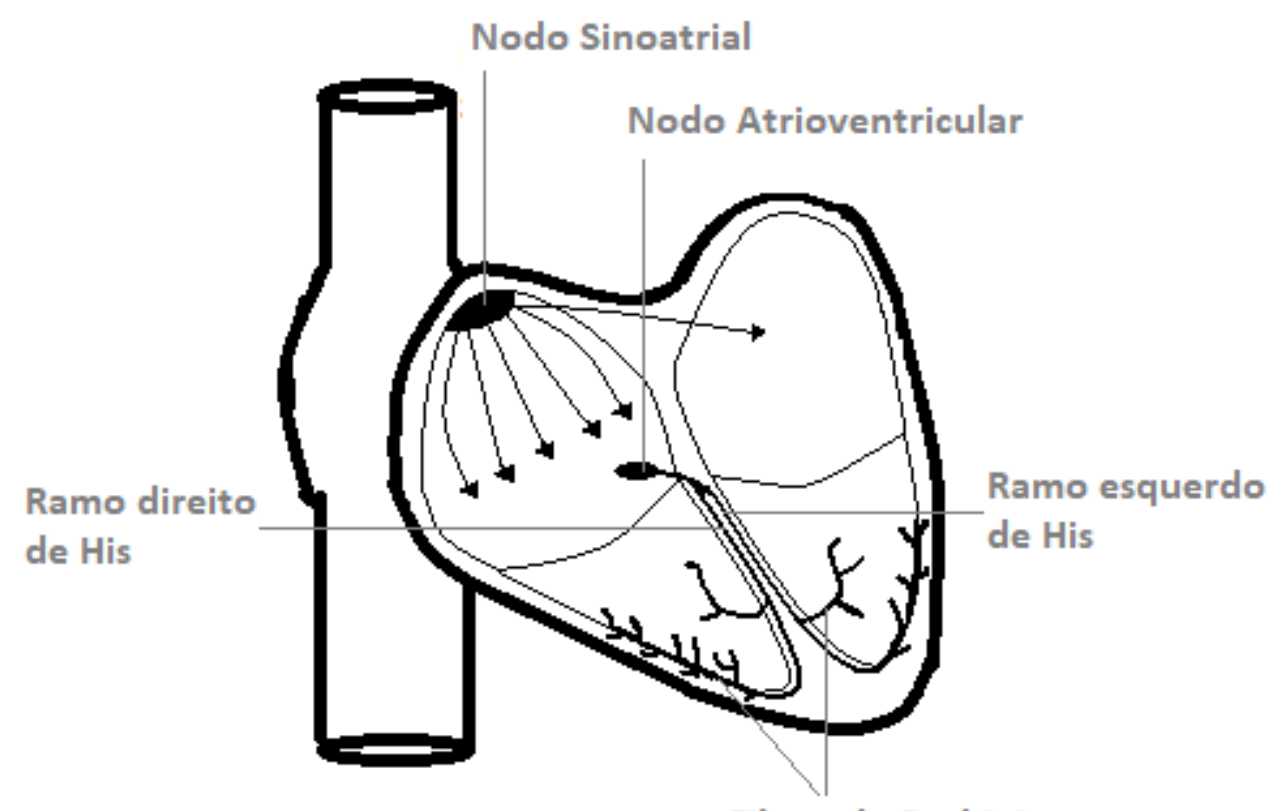

Fibras de Purkinje

Fonte: autor 


\section{A.2 Eletrocardiograma}

A corrente elétrica que se propaga do coração para os tecidos adjacentes até a superfície do corpo devido à propagação dos potenciais de ação no coração pode ser registrada por meio de eletrodos colocados sobre a pele em lados opostos do coração. Esse registro é conhecido como eletrocardiograma (ECG), que normalmente é composto pela onda P, complexo QRS e pela onda T. (Guyton; Hall, 2011)

A onda $P$ é causada pela despolarização que se difunde pelos átrios. Em consequência da despolarização dos ventrículos aparecem as ondas QRS. A onda T representa a fase de repolarização dos ventrículos (Guyton; Hall, 2011).

A figura 32 ilustra um eletrocardiograma normal, obtida do arquivo 26 do banco de dados Long-Term Atrial Fibrillation da Physionet.

Figura 32 - Ondas típicas do eletrocardiograma normal, com as marcações em verde e a linha de base em azul.

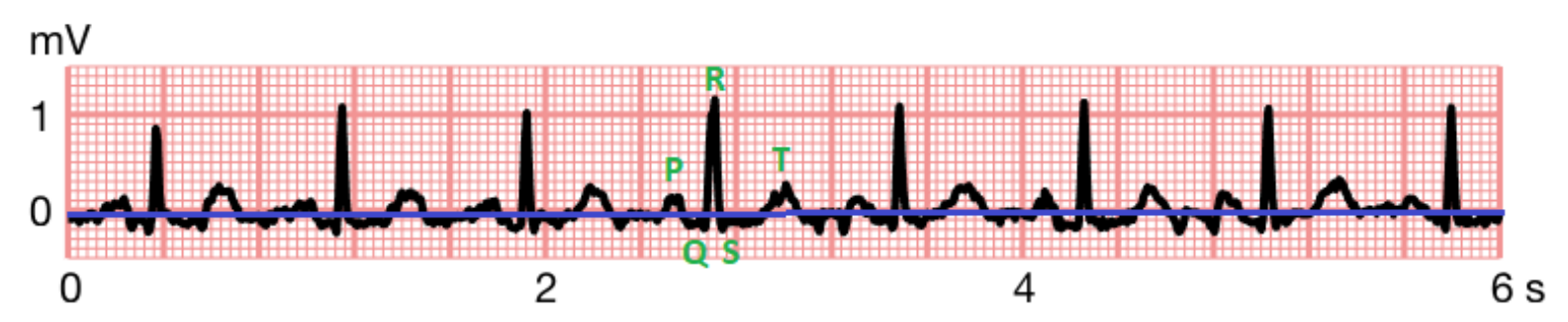

A figura 33 ilustra a captação do sinal de ECG. As derivações precordiais (V1, V2, V3, V4, V5 e V6) estão representadas por círculos de cor azul. As derivações bipolares (I, II, III) estão representadas em preto. 
Figura 33 - Representação da captação do sinal de ECG.

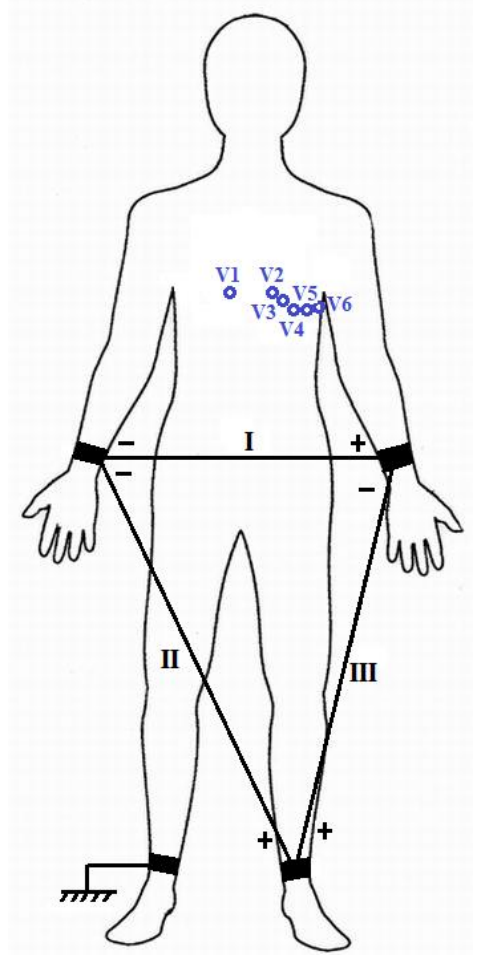

Fonte: Próprio autor.

Além da irregularidade da frequência cardíaca, a existência ou deformação da onda $P$ é um dos fatores que permite a identificação da FA. As derivações II e V1 apresentam a onda $\mathrm{P}$ mais destacada em relação às outras derivações, por isso são as mais utilizadas para a detecção da FA.

A Figura 34 ilustra um ECG, um tacograma (sequência dos intervalos de tempo entre batimentos sucessivos no ECG) e as frequências cardíacas para um caso normal. 
Figura 34: Sinais de a) eletrocardiograma, b) tacograma e c) frequência cardíaca para caso normal. O intervalo RR (intervalo entre dois picos $R$ consecutivos) médio \pm desviopadrão é de 0,7732 $\mathrm{s} \pm 0,0068 \mathrm{~s}$; a mediana dos intervalos é de 0,7734 s e a razão entre máximo e mínimo intervalos $R R$ é de 1,0412. A frequência cardíaca média \pm desvio-padrão é de 77,6082 b.p.m. $\pm 0,6804$ b.p.m. e a mediana é de 77,5758 b.p.m.
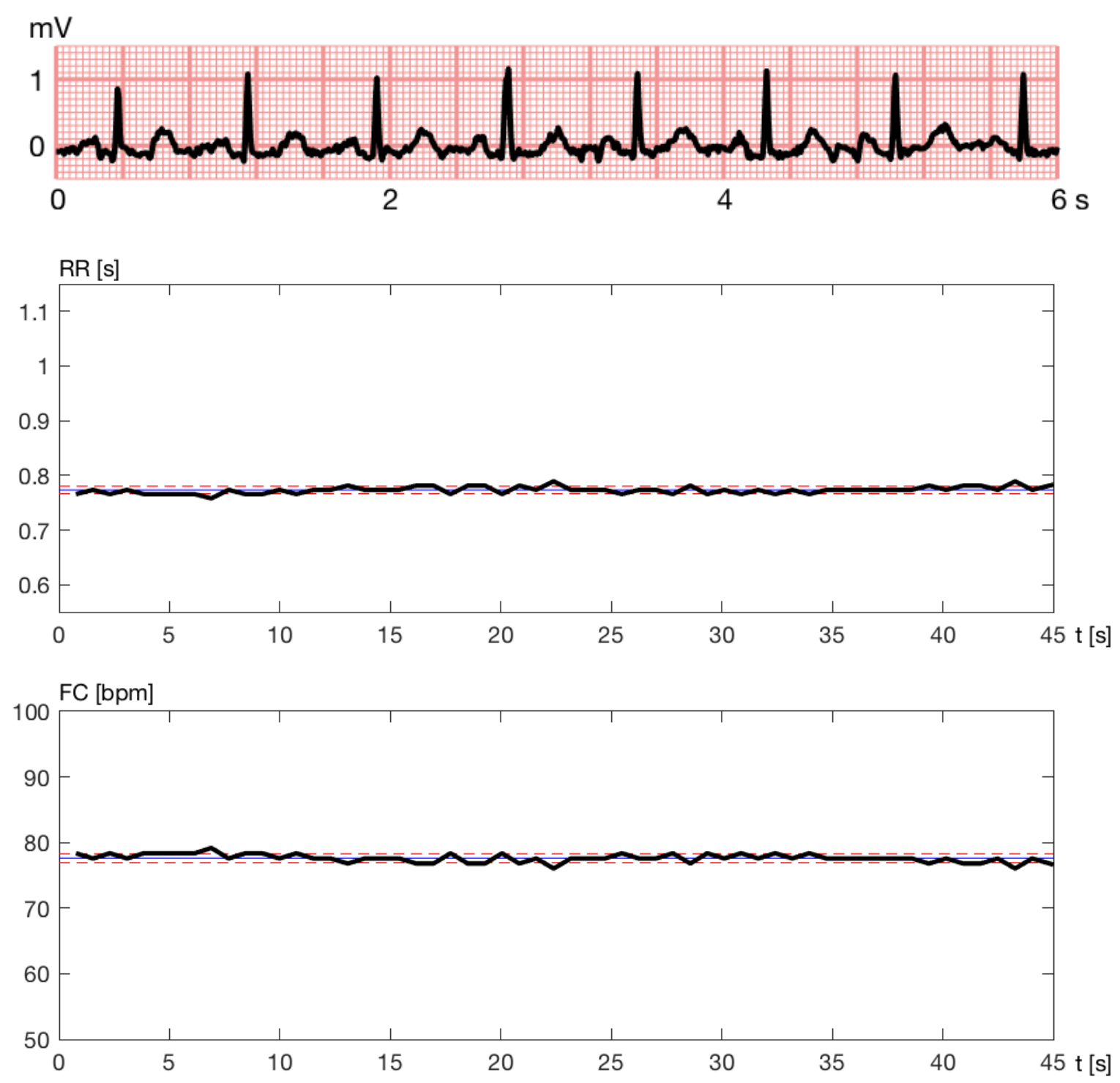

Fonte: Próprio autor. 


\section{A.3 Fibrilação Atrial}

A fibrilação atrial (FA) é uma arritmia cardíaca caracterizada no eletrocardiograma por oscilações de baixa amplitude da linha de base, denominada ondas $F$, pela elevação e pela irregularidade da frequência cardíaca, além da desorganização da despolarização atrial (Neto, 2012; Sociedade Brasileira de Cardiologia, 2009).

A detecção da FA é importante, porque a FA aumenta os riscos de ocorrer um acidente vascular encefálico devido ao aumento da chance de formação de trombos com o fluxo de sangue turbulento e incapacidade de esvaziar o sangue totalmente do apêndice atrial causados pela irregularidade dos batimentos cardíacos. (Nesheiwat et al, 2020; Ludhwani; Wieters, 2020).

Ela pode ser assintomática como também pode apresentar os sintomas como dor no peito, palpitações, frequência cardíaca elevada, dispneia, náusea, tontura, diaforese e fatiga. (Nesheiwat et al, 2020; Ludhwani; Wieters, 2020). O mecanismo da FA está relacionado com a interação entre o gatilho e foco ectópico e o substrato. O gatilho é o responsável pelo início da arritmia e o substrato, que pode ser alterações fibróticas ou cicatrizes, permite a manutenção da arritmia. (Ludhwani; Wieters, 2020).

Os gatilhos são formados quando o potencial de ação induz pós-despolarização que supera a recuperação da repolarização. As pós-despolarizações podem causar extrasístoles, que ao contato com o miocárdio no período refratário geram bloqueios elétricos. Com o substrato (alterações fibróticas ou cicatrizes) são gerados circuitos de re-entradas que geram novos impulsos criando novos circuitos de reentrada que ajudam a manter a arritmia. (Ludhwani; Wieters, 2020).

O local mais comum de formação dos gatilhos são as veias pulmonares que apresentam células de miocárdio com período refratário menor. Por isso a isolação da veia pulmonar durante a ablação de cateter pode ser usada para tentar evitar o desenvolvimento da fibrilação atrial. Outra fonte de gatilhos e substrato são as outras arritmias como flutter atrial e contração atrial prematura que podem gerar alterações estruturais e elétricas. Outras doenças e fatores de risco que podem alterar a estrutura do coração frequentemente estão associadas a FA. (Ludhwani; Wieters, 2020). 
A Figura 35 ilustra um ECG, um tacograma e as frequências cardíacas para um caso com fibrilação atrial.

Figura 35: Sinais de a) eletrocardiograma, b) tacograma e c) frequência cardíaca para caso com fibrilação atrial. O intervalo RR médio \pm desvio-padrão é de 0,8619 s \pm 0,1247 s; a mediana dos intervalos é igual a 0,8594 s e a razão entre o máximo

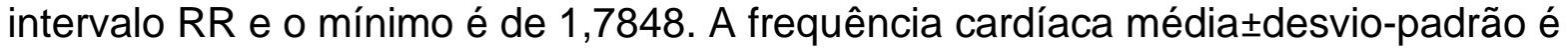
de 71,1225 b.p.m. $\pm 10,7538$ b.p.m. e a mediana é de 69,8182 b.p.m.
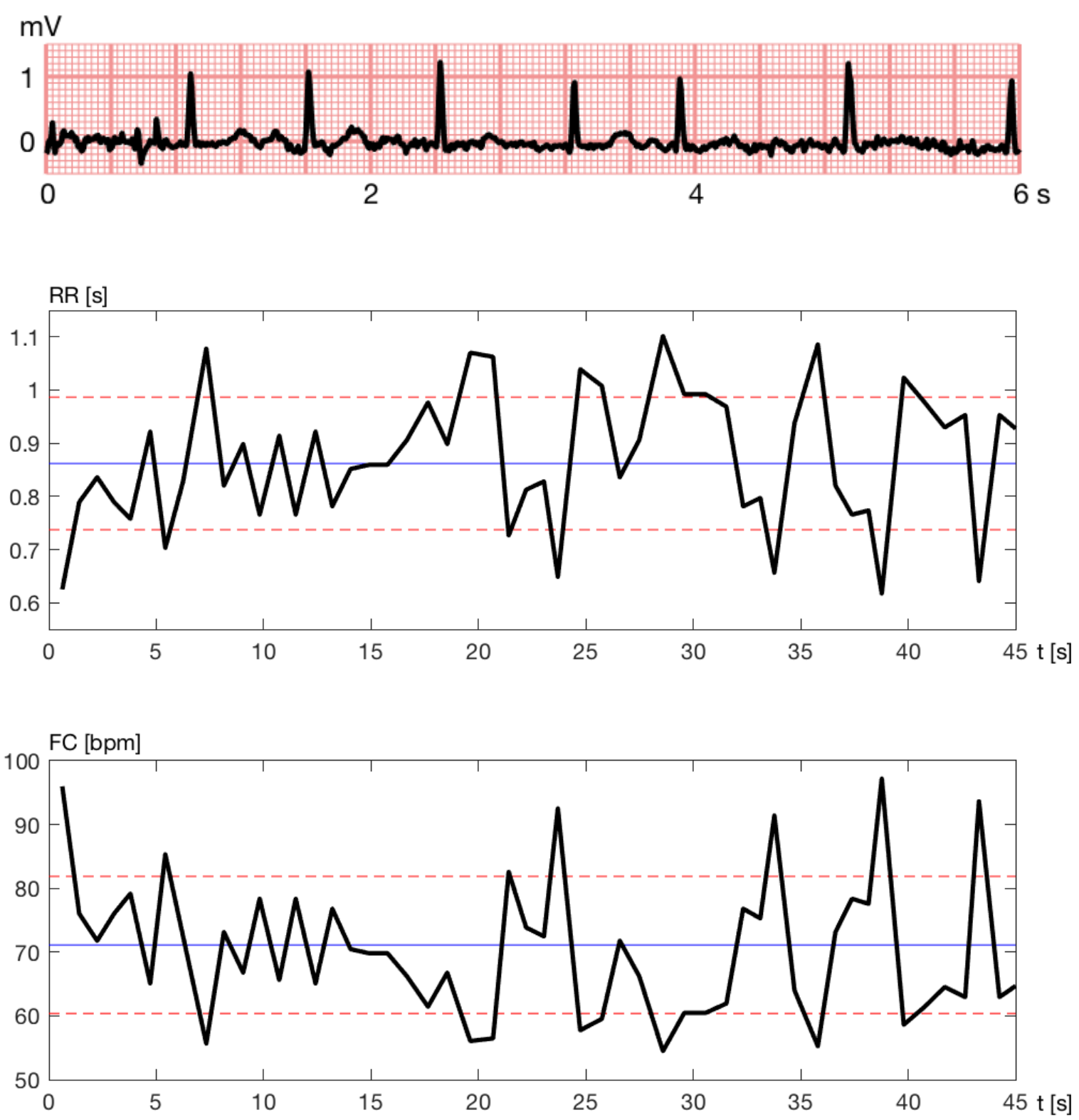

Fonte: Próprio autor. 
$\mathrm{Na}$ Figura 34, os sucessivos intervalos RR têm valores semelhantes, no caso do eletrocardiograma normal. No entanto, para o eletrocardiograma com fibrilação atrial, os intervalos entre picos $\mathrm{R}$ apresentam diferenças perceptíveis visualmente. Por exemplo, os dois últimos intervalos RR são muito mais longos do que o antepenúltimo intervalo da figura 35 .

No caso normal, o intervalo RR médio é de $0,7732 \mathrm{~s}$, enquanto que no caso com fibrilação atrial, o valor médio é 0,8619 s. No entanto, o maior contraste está nos valores de desvio-padrão. No caso normal, o desvio padrão de 59 intervalos RR é de $0,0068 \mathrm{~s}$, enquanto que para o caso de fibrilação atrial esse desvio é de $0,1247 \mathrm{~s}$. A diferença entre os dois casos fica também muito evidente na razão entre o máximo e o mínimo intervalo RR: 1,0412 no caso normal e 1,7848 no caso com fibrilação atrial.

As figuras 34c e 35c permitem observar a variabilidade da frequência cardíaca nos casos normal e com fibrilação atrial respectivamente. Para a frequência cardíaca normal, o valor médio é de 77,6082 b.p.m. e o desvio-padrão de 0,6804 b.p.m. No caso com fibrilação atrial, a frequência cardíaca média de 71,1225 b.p.m. é relativamente próxima ao valor do caso normal. No entanto, o desvio-padrão é de 10,7538 b.p.m. que é muito diferente do normal.

\section{A.4 Análise de componentes principais (PCA)}

A análise de componentes principais consiste em reduzir a dimensionalidade realizando uma transformação dos dados para novos parâmetros chamados de componentes principais. A transformação é feita de forma a maximizar a variação dos dados, resultando em componentes não correlacionados e ordenados de acordo com a variação em ordem decrescente. [Jolliffe, 2002] 
Figura 36: Gráfico de representação dos componentes principais.

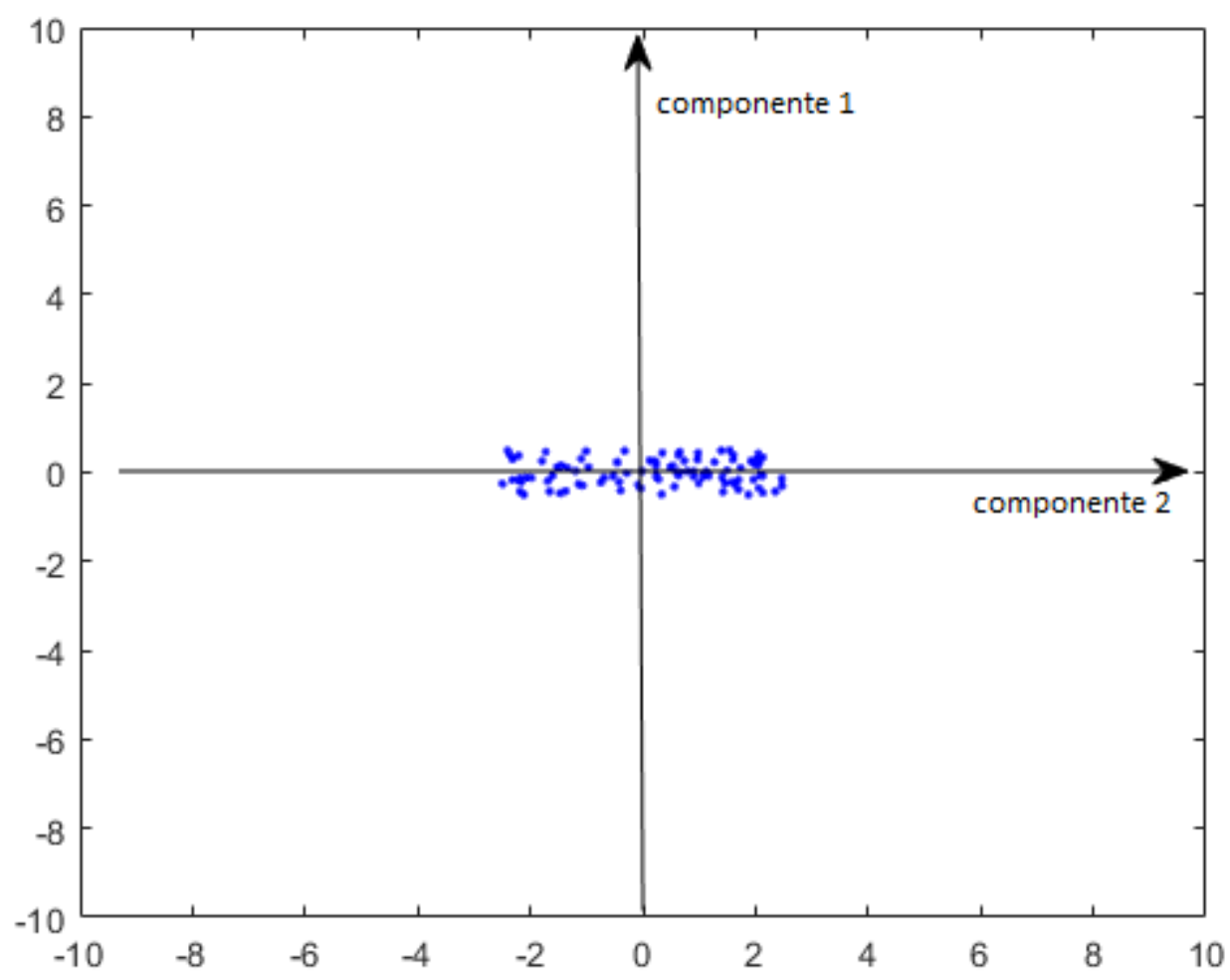

Fonte: Próprio autor.

Considera-se $\mathbf{x}$ um vetor de quantidade $p$ de parâmetros e $\Sigma$ a sua matriz de covariância.

Para determinar os componentes principais, são procuradas as funções lineares $\boldsymbol{\alpha}_{n}^{\mathrm{T}} \mathbf{x}$ não correlacionadas, que apresentam a máxima variância, da forma

$$
\boldsymbol{\alpha}_{n}^{\mathrm{T}} \mathbf{x}=\sum_{j=1}^{p} \alpha_{n, j} x_{j},
$$

em que $\boldsymbol{\alpha}_{n}$ é um vetor com $p$ constantes $\alpha_{n, 1}, \alpha_{n, 2}, \ldots, \alpha_{n, p} \operatorname{com} \mathrm{n}=1,2, \ldots, p$.

Os vetores $\boldsymbol{\alpha}_{n}$ são obtidos maximizando a variância

$$
\operatorname{var}\left(\boldsymbol{\alpha}_{n}^{\mathrm{T}} \mathbf{x}\right)=\boldsymbol{\alpha}_{n}^{\mathrm{T}} \sum \boldsymbol{\alpha}_{n},
$$


sujeita a restrição

$$
\boldsymbol{\alpha}_{n}^{\mathrm{T}} \boldsymbol{\alpha}_{n}=1
$$

Este problema pode ser resolvido com o método dos multiplicadores de Lagrange. Os multiplicadores de Lagrange ótimos $(\lambda)$ maximizam a função

$$
Q(\lambda)=\boldsymbol{\alpha}_{n}^{\mathrm{T}} \sum \boldsymbol{\alpha}_{n}-\lambda\left(\boldsymbol{\alpha}_{n}^{\mathrm{T}} \boldsymbol{\alpha}_{n}-1\right)
$$

o que resulta em

$$
\left(\sum-\lambda \mathbf{I}_{\mathrm{p}}\right) \boldsymbol{\alpha}_{n}=0
$$

em que $\mathbf{I}_{\mathrm{p}}$ é a matriz identidade de dimensão pxp. Tem-se que $\lambda$ é um autovalor de $\sum$ e $\boldsymbol{\alpha}_{n}$ é o autovetor correspondente. O autovetor $\boldsymbol{\alpha}_{1}$ corresponde ao maior autovalor de $\sum$.

O k-ésimo componente principal é dado por $\boldsymbol{z}_{\mathrm{k}}=\boldsymbol{\alpha}_{k}^{\mathrm{T}} \mathbf{x}$, em que $\boldsymbol{\alpha}_{k}$ é um autovetor de $\sum$ correspondente ao seu k-ésimo maior autovalor $\lambda_{\mathrm{k}}$. 